\title{
Cortical dynamics of three-dimensional form, color, and brightness perception: II. Binocular theory
}

\author{
STEPHEN GROSSBERG \\ Boston University, Boston, Massachusetts
}

\begin{abstract}
A real-time visual processing theory is developed to explain how three-dimensional form, color, and brightness percepts are coherently synthesized. The theory describes how several fundamental uncertainty principles which limit the computation of visual information at individual processing stages are resolved through parallel and hierarchical interactions among several processing stages. The theory hereby provides a unified analysis and many predictions of data about stereopsis, binocular rivalry, hyperacuity, McCollough effect, textural grouping, border distinctness, surface perception, monocular and binocular brightness percepts, filling-in, metacontrast, transparency, figural aftereffects, lateral inhibition within spatial frequency channels, proximityluminance covariance, tissue contrast, motion segmentation, and illusory figures, as well as about reciprocal interactions among the hypercolumns, blobs, and stripes of cortical areas V1, V2, and V4. Monocular and binocular interactions between a Boundary Contour (BC) System and a Feature Contour (FC) System are developed. The BC System, defined by a hierarchy of oriented interactions, synthesizes an emergent and coherent binocular boundary segmentation from combinations of unoriented and oriented scenic elements. These BC System interactions instantiate a new theory of stereopsis and of how mechanisms of stereopsis are related to mechanisms of boundary segmentation. Interactions between the BC System ind the FC System explain why boundary completion and segmentation processes become binocular at an earlier processing stage than do color and brightness perception processes. The new stereopsis theory includes a new model of how chromatically broadband cortical complex cells can be adaptively tuned to multiplex information about position, orientation, spatial frequency, positional disparity, and orientational disparity. These binocular cells input to spatially short-range competitive interactions (within orientations and between positions, followed by between orientations and within positions) that initiate suppression of binocular double images as they complete boundaries at scenic line ends and corners. The competitive interactions interact via both feedforward and feedback pathways with spatially long-range-oriented cooperative gating interactions that generate a coherent, multiple-scale, three-dimensional boundary segmentation as they complete the suppression of double-image boundaries. The completed BC System boundary segmentation generates output signals, called filling-in generators (FIGs) and filling-in barriers (FIBs), along parallel pathways to two successive FC System stages: the monocular syncytium and the binocular syncytium. FIB signals at the monocular syncytium suppress monocular color and brightness signals that are binocularly inconsistent and select binocularly consistent, monocular FC signals as outputs to the binocular syncytium. Binocular matching of these FC signals further suppresses binocularly inconsistent color and brightness signals. Binocular FC contour signals that survive these multiple suppressive events interact with FIB signals at the binocular syncytium to fill-in a multiplescale representation of form-and-color-in-depth. To achieve these properties, distinct syncytia correspond to each spatial scale of the BC System. Each syncytium is composed of opponent subsyncytia that generate output signals through a network of double-opponent cells. Although composed of unoriented wavelength-sensitive cells, double-opponent networks detect oriented properties of form when they interact with FIG signals, yet also generate nonselective properties of binocular rivalry. Electrotonic and chemical transmitter interactions within the syncytia are formally akin to interactions in $\mathrm{H} 1$ horizontal cells of turtle retina. The cortical syncytia are hypothesized to be encephalizations of ancestral retinal syncytia. In addition to double-opponent-cell networks, electrotonic syncytial interactions, and resistive gating signals due to BC System outputs, the FC System processes also include habituative transmitters and non-Hebbian adaptive filters that maintain the positional and chromatic selectivity of FC interactions. Alternative perceptual theories are evaluated in light of these results. The theoretical circuits provide qualitatively new design principles and architectures for computer vision applications.
\end{abstract}

This work was supported in part by grants from the Air Force Office of Scientific Research (AFOSR 85-0149 and AFOSR F49620-86-C-0037), the Army Research Office (ARO DAAG-29-85-K-0095), and the National Science Foundation (NSF IST-8417756). Thanks are extended to Cynthia Suchta for her valuable assistance in the preparation of the manuscript and illustrations. The author's address is: Center for Adaptive Systems, Boston University, 111 Cummington Street, Boston, MA 02215. 


\section{Introduction: From Filling-In Resonant \\ Exchange to Boundary-Feature Tradeoff}

The second of this pair of articles builds a neural theory of 3-D form perception using as a foundation the mechanisms of monocular form perception that were summarized and applied in the first (Grossberg, 1987b). A macrocircuit of this 3-D theory is shown in Figure 1. One of the main purposes of the article is to suggest how mechanisms of stereopsis and of boundary segmentation work together to generate an emergent percept of formand-color in-depth. Another general goal of the article is to explain why mechanisms of boundary segmentation within the Boundary Contour System become binocular at an earlier processing stage than do the mechanisms of Feature Contour extraction and filling-in within the Feature Contour System (Figure 1). Yet another contribution is to show how networks of unoriented doubleopponent wavelength-sensitive cells can compute oriented form-sensitive properties when they interact with boundary contour signals.

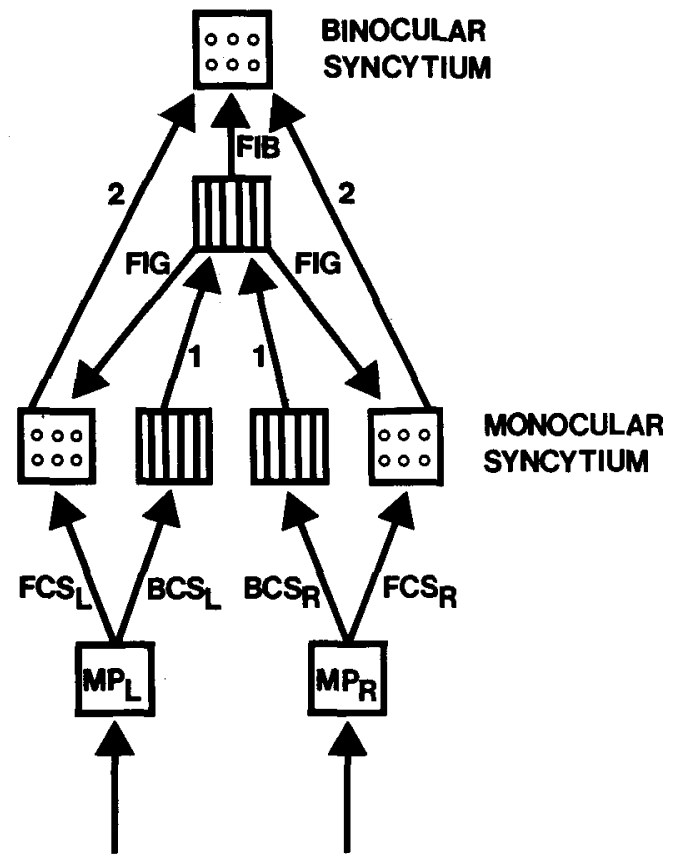

Figure 1. Macrocircuit of monocular and binocular interactions within the Boundary Contour System (BCS) and the Feature Contour System (FCS): Left and right monocular preprocessing stages $\left(M P_{L}\right.$ and $M P_{R}$ ) send parallel monocular inputs to the BCS (boxes with vertical lines) and the FCS (boxes with three pairs of circles). The monocular $\mathrm{BCS}_{\mathrm{L}}$ and $\mathrm{BCS}_{\mathrm{R}}$ interact via bottom-up pathways labeled 1 to generate a coherent binocular boundary segmentation. This segmentation generates output signals called filling-in generators (FIGs) and filling-in barriers (FIBs). The FIGs input to the monocular syncytia of the FCS. The FIBs input to the binocular syncytia of the FCS. The text describes how inputs from the MP stages interact with FIGs at the monocular syncytia to selectively generate binocularly consistent feature-contour signals along the pathways, labeled 2, to the binocular syncytia. The text also describes how these monocular feature contour signals interact with FIB signals to generate a multiple-scale representation of form-and-color-in-depth within the binocular syncytia.
As noted in the first article (Grossberg, 1987b), the present theory is a synthesis of two previous theories. Both theories got their start through an analysis of binocular perceptual data that required a combination of cooperative and competitive interactions for their analysis (Grossberg, 1980, 1981, 1983b). These data suggested how a single contour-sensitive process could control featural filling-in. This filling-in process was called filling-in resonant exchange, or FIRE. Properties of the FIRE process enabled qualitative explanations and quantitative computer simulations of binocular perceptual data to be made (Cohen \& Grossberg, 1984a, 1984b; Grossberg, 1983a). However, the quantitative analysis of FIRE properties also led to the realization that a distinct type of filling-in-called diffusive, or electrotonic, filling-inwas needed to preprocess visual inputs before they were transformed by the FIRE itself.

This insight opened the way to distinguishing between two distinct types of contour-sensitive processes-the Boundary Contour (BC) System and the Feature Contour (FC) System (Grossberg, 1983a, 1984). At first, the BC System and the FC System were used to analyze data about monocular percepts, as in Grossberg (1987b). However, the theory as a whole then used two different types of filling-in-one monocular (diffusive) and the other binocular (FIRE)-and two different types of cooperativecompetitive (CC) interactions-one monocular (CC loop) and the other binocular (FIRE). In addition, as the FIRE theory began to be used to analyze complex twodimensional (2-D) images, the analysis seemed to become unnecessarily complicated.

These inelegances ultimately focused attention upon the following demanding problem: How could the FIRE process be replaced by a binocular theory of the BC System and FC System while preserving all of the good properties of the FIRE process within a unified theory with an expanded predictive range? Why did the FIRE process work so well if it could be replaced in such a fashion? The present theory is the result of this quest, and satisfies the above requirements. The theory suggests, moreover, that the FIRE explanations were all qualitatively, if not mechanistically, correct.

I will not redevelop in this article all of the data that were analyzed using properties of the FIRE process in Cohen and Grossberg (1984a, 1984b) and Grossberg (1983b). Instead, I will cut a steep path to neural architectures. Along the way, I will sketch explanations of some binocular data that the FIRE theory could explain to show how previous explanations carry over to the present theory. I will also develop explanations of several important data bases that the FIRE theory could not explain.

\section{Two Types of Binocular Rivalry:}

The Kaufman Stereogram

Just as percepts of neon color spreading provide a vivid guide to conceptualizing key monocular processes (Grossberg, 1987b; Grossberg \& Mingolla, 1985a), percepts of binocular rivalry provide crucial clues concerning the 
mechanisms of binocular processing. In particular, two mechanistically distinct types of binocular rivalry can be identified by comparing properties of rivalry data with formal properties of the BC System circuits depicted in Figures 2 and 3.

Figure 2 schematizes early stages of filtering and shortrange competition within the monocular BC System. Figure 3 joins these early stages to a later stage of longrange cooperation. Together, the entire monocular BC System is broken into two subsystems (Figure 3): an OC filter and a CC loop, which were used in Grossberg (1987b) to analyze properties of monocular data. The discussion below of binocular rivalry data motivates how these monocular mechanisms can be generalized to the binocular case. The possibility of carrying out such a generalization cannot be taken for granted. In particular, the competition and cooperation mechanisms shown in Figures 2 and 3 are new to our theory, and possess properties that are essential for carrying out the generalization from monocular to binocular processing.

The Kaufman (1974) stereogram illustrates the first type of binocular rivalry (Figure 4). In this stereogram, the

\section{TO COOPERATION}

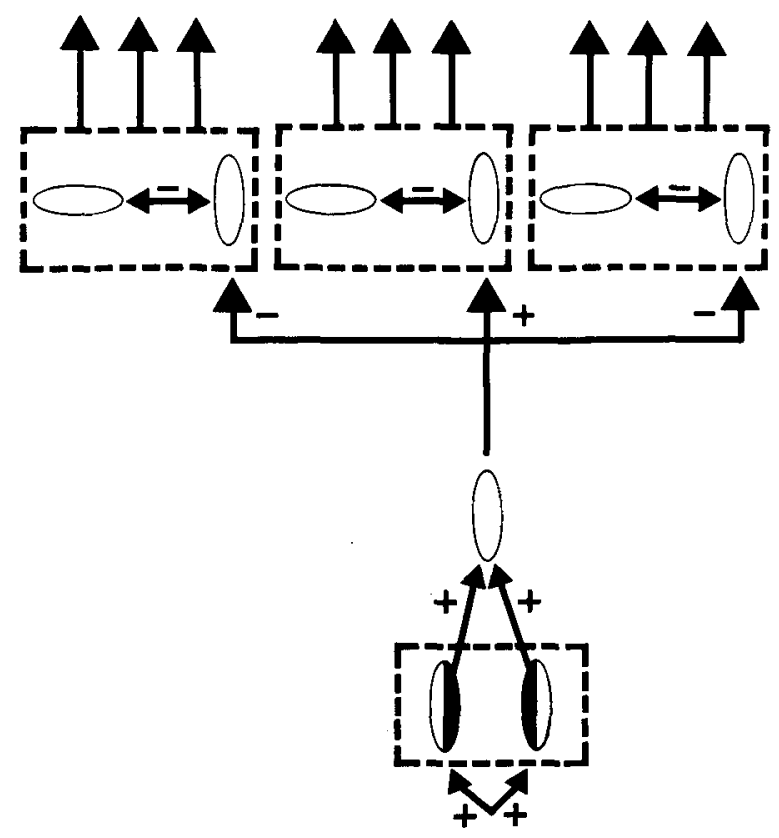

Figure 2. Farly stages of BC System processing: At each position exist cells with elongated receptive fields of various sizes which are sensitive to orientation, amount-of-contrast, and direction-ofcontrast. Pairs of such cells sensitive to like orientation but opposite directions-of-contrast (lower dashed box) input to cells that are sensitive to orientation and amount-of-contrast but not to directionof-contrast (white ellipses). These cells, in turn, excite like-oriented cells corresponding to the same position and inhibit like-oriented cells corresponding to nearby positions using the first competitive stage. At the second competitive stage (upper dashed boxes), cells corresponding to the same position but different orientations inhibit each other via a push-pull competitive interaction.

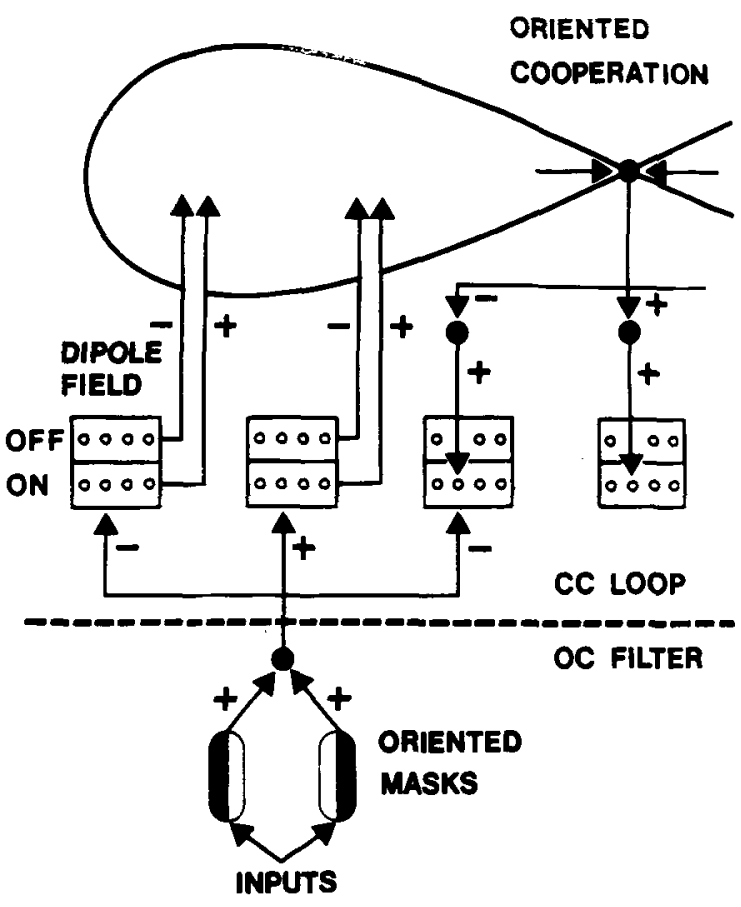

Figure 3. Circuit diagram of the BC System: Inputs activate oriented masks of opposite direction-of-contrast which cooperate at each position and orientation before feeding into an on-center offsurround interaction. This interaction excites like orientations at the same position and inhibits like orientations at nearby positions. The affected cells are on-cells within a dipole field. On-cells at a fixed position compete among orientations. On-cells also inhibit of -cells that represent the same position and orientation. Off-cells at each position, in turn, compete among orientations. Both on-cells and off-cells are tonically active. Net excitation of an on-cell excites a similarly oriented receptive field of a cooperative bipole cell at a location corresponding to that of the on-cell. Net excitation of an off-cell inhibits a similarly oriented receptive field of a bipole cell at a location corresponding to that of the off-cell. Thus, bottom-up excitation of a vertical on-cell, by inhibiting the horizontal on-cell at that position, disinhibits the horizontal off-cell at that position, which in turn inhibits (almost) horizontally oriented cooperative receptive fields that include its position. Suficiently strong net positive activation of both receptive fields of a bipole cell enables it to generate feedback via an on-center ofi-surround interaction among like-oriented cells. On-cells that receive the most favorable combination of bottom-up signals and top-down signals generate the emergent perceptual grouping.

left picture is constructed from $45^{\circ}$-oblique dark parallel lines bounded by an imaginary square that is surrounded by $135^{\circ}$-oblique lighter parallel lines. The right picture is constructed from $135^{\circ}$-oblique dark parallel lines bounded by an imaginary square whose position in the picture is shifted relative to the square in the left picture. This imaginary square is surrounded by $45^{\circ}$-oblique lighter parallel lines. When these pictures are viewed through a stereoscope, a single fused square is seen in depth relative to the background. Superimposed upon the continuous percept of a square in depth is a rivalrous percept due to the dark oblique lines. At any given moment, an observer can perceive either a $45^{\circ}$-oblique dark line 

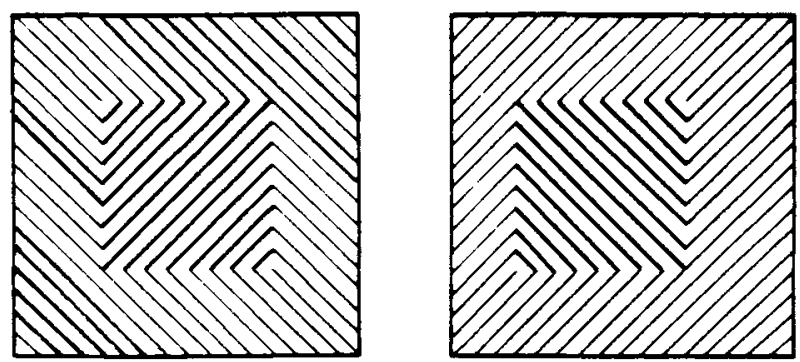

Figure 4. The Kaufman stereogram induces a percept of a square in depth even while the perpendicular line patterns are rivalrous. From Sight and Mind: An Introduction to Visual Perception by L. Kaufman, 1974, New York: Oxford University Press. Copyright 1974 by Oxford University Press. Reprinted by permission.

or a $135^{\circ}$-oblique dark line at a given position, but not both. The percept switches intermittently between these mutually perpendicular lines.

This rivalrous percept illustrates that images that can be clearly seen under monocular viewing conditions may not be visible under binocular viewing conditions when the other eye is viewing a discordant image. These mutually perpendicular lines tend to pop into and out of conscious perception with coherent properties: If several short $45^{\circ}$-oblique line elements start to be perceived, then the whole $45^{\circ}$-oblique line segment between them is also often perceived.Thus, binocular rivalry provides striking illustrations of how binocular grouping processes regulate visible percepts of 3-D form. Further consideration of this percept of simultaneous fusion and rivalry implicates binocular versions of the mechanisms summarized in Figures 2 and 3.

\section{Interaction Between Segmentation and \\ Stereopsis Mechanisms Within the CC Loop}

Percepts of the Kaufman stereogram illustrate one sense in which multiple spatial scales are operative during binocular perception: A square cn be perceived continuously in depth, whereas perpendicular lines are rivalrous at the same positions in perceptual space. The Kaufman percept also emphasizes the intimate linkage that exists between mechanisms of segmentation and mechanisms of stereopsis. Although a square is perceived continuously in depth, neither image in Figure 4 contains a square. A square is synthesized from each image as an emergent illusory boundary. This illusory boundary interpolates the corner discontinuities at the ends of the oblique line segments in each image. Grossberg (1987b) described a mechanism for generating such an emergent illusory boundary at line ends and corners. This process is initiated by end cuts at the second competitive stage of the $O C$ filter. These end cuts interact via the long-range cooperative process of the CC loop to generate a sharp emergent boundary from the fuzzy bands of end cuts that exist at each line corner.

Given this linkage with CC loop mechanisms, the percept of a square in depth suggests that these mechanisms are binocular. In particular, the imaginary squares of the two images are positionally disparate with respect to the frame of each image. Consequently, when each image of the stereogram is monocularly viewed, each square boundary emerges at a different perceptual location. In contrast, under binocular viewing conditions, only a single fused square boundary is perceived. Thus, the CC loop must combine the pair of positionally disparate, monocular emergent boundaries into a single, fused binocular boundary. In order for this to occur, a binocular matching process must occur within the CC loop. This binocular matching process operates upon the emergent boundaries generated by the images to each eye, rather than upon the individual local contrasts within these images. The process whereby a single fused square in depth is generated from pairs of emergent boundaries thus combines mechanisms of segmentation with mechanisms of stereopsis.

Of course, there exist many images whose emergent boundary disparities covary with the disparities of their image contrasts. The Kaufman stereogram reminds us, however, that this is just as often false. When it is false, emergent segmentations can override local image disparities during the synthesis of a 3-D percept, as has been shown by a number of experimental studies (Ramachandran \& Nelson, 1976; Tausch, 1953; Wilde, 1950).

\section{The Second Competitive Stage is Binocular: Correlated Emergence and Suppression of Coherent Boundaries}

Finer conclusions can be drawn by considering the rivalry that is perceived when the Kaufman stereogram is viewed. This rivalry occurs between perpendicular orientations at each position of perceptual space (Figure 4). This is the very sort of competition that occurs at the second competitive stage of the CC loop (Figure 2). The linking hypothesis that the second competitive stage is responsible for the orientational competition that occurs during rivalry will be used to help explain a large binocular data base below. This linking hypothesis provides my first example of the fact that the segmentation laws governing the CC loop, which were derived from an analysis of monocular data, generalize directly to the binocular case.

The linking hypothesis implies that the second competitive stage is binocular, so that perpendicular boundary signals from both eyes can compete by the time they reach this stage. The linking hypothesis also helps to explain how whole boundary segments can coherently pop into and out of consciousness. Suppose that several collinear $45^{\circ}$-oriented cells at the second competitive stage get simultaneously activated by the image to one eye. These cells can excite like-oriented bipole cells at the cooperative stage of the CC loop (Figure 3). The activated bipole cells can use cooperative feedback to excite $45^{\circ}$ oriented cells at the second competitive stage at positions between the originally excited cells. These newly excited cells can inhibit $135^{\circ}$-oriented cells at their positions via the orientational competition that takes place at the second competitive stage (Figure 2). Thus, the fact that 
boundary completion is an inwardly directed process between pairs of inducing elements enables whole boundary segments to emerge in response to spatially separated inducers. The fact that orientational competition is engaged by the same cooperative feedback that controls boundary completion shows how the emergence of a given boundary can cause the suppression of perpendicular boundaries that intersect the same perceptual locations.

In summary, if a sufficient number of like orientations from one scenic image can momentarily suppress the perpendicular orientations from the other scenic image, then these winning orientations can collinearly cooperate to complete the oriented boundary at intervening positions. The oriented cooperative feedback can inhibit any perpendicularly oriented cells at the intervening positions by using the second competitive stage. This combination of competition between orientations at each position and collinear cooperation between positions helps to explain why whole segments of the rivalrous interior edges of the square seem to pop coherently into and out of conscious perception.

\section{The Kulikowski Stereogram}

The second type of binocular rivalry is exemplified by the percepts seen in response to the Kulikowski (1978) stereograms. The Kulikowski stereograms consist of two pairs of pictures which differ in their spatial frequencies. Each picture is bounded by an identical frame, which includes a pair of short vertical reference lines to help viewers fuse the frames binocularly. In one pair of pictures, spatially blurred alternating black and white vertical bars of a fixed spatial frequency are $180^{\circ}$ out of phase (Figure 5a). In the other pair of pictures (Figure 5b), sharp black and white vertical bars of the same spatial extent are $180^{\circ}$ out of phase. The latter pair of pictures contains high spatial-frequency components (edges) as well as the low spatial-frequency components of the first pair.

During binocular viewing, subjects can fuse the two spatially blurred pictures. They perceive the fused image continuously in depth relative to the fused images of the

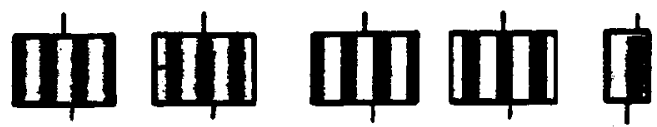 IIII
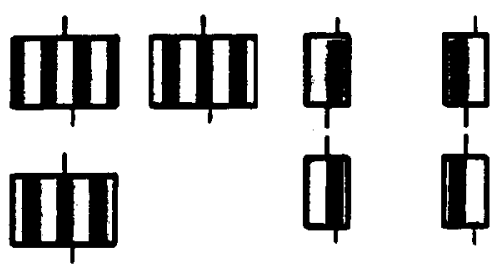 \\ $a$ \\ $b$ \\ 6}

Figure 5. The Kulikowski stereograms illustrate the action of multiple spatial-frequency-sensitive scales during simultaneous fusion and rivalry: (a) Sinusoidal gratings in antiphase can be fused to yield a percept of a grating in depth. (b) Bar gratings in antiphase yield a percept of depth even though their edges are rivalrous. (c) Similar percepts are seen when single sinusoids or bars are viewed. From "Limit of Single Vision in Stereopsis Depends on Contour Sharpness" by J. J. Kulikowski, 1978, Nature, 275, 126-127. Copyright 1978 by Macmillan. Reprinted by permission. two frames. Subjects experience a more complex percept when they view the stereogram composed from out-ofphase sharp bars. As in the case of the viewing of blurred bars, a fused form-in-depth is again perceived continuously. However, superimposed upon the fused percept of form-in-depth is a percept of binocular rivalry. The spatially out-of-phase edges of the bars in the left and right images are rivalrous and appear to pop alternately into and out of conscious perception.

\section{Simultaneous Fusion and Rivalry: Multiple Spatial Scales}

This percept of simultaneous fusion and rivalry illustrates the operation of multiple spatial scales: The lower spatial frequency components of the two images can be binocularly fused into a continuously seen percept in depth at the same time that the high spatial frequency edges are seen to be rivalrous. Thus, the high spatial frequency scales that process the edges cannot fuse these image properties at the same disparity at which the low spatial frequency scales can fuse the low spatial frequency contents of the two images. In contrast, the frames of both images can be fused by all spatial scales because the frames can be spatially aligned with respect to both eyes. Thus, the Kulikowski stereogram provides visible evidence of the classical fact (Julesz, 1971) that a relative depth percept can be generated when different parts of two images are fused by different combinations of binocular spatial scales.

The relative depth percept that is generated by the Kaufman stereogram (Figure 4) can also be analyzed from this perspective. As described in Section 3, the formation of an emergent boundary around the illusory square region is initiated by end cuts at the corners where the oblique line ends of the square join its surround. Due to the horizontal disparity of the end cuts formed in response to the left and right images, not all of the BC System spatial scales can form fused images of these emergent boundaries. In contrast, the scenic edges that frame the image pair have zero disparity with respect to each other and can thus form fused boundary responses within all spatial scales of the BC System. The selective activation of a subset of BC System scales by the disparate image figures provides the basis for a relative depth difference of the figure with respect to the ground.

As described in Section 3, end cuts, in themselves, are insufficient to generate an illusory square boundary. The CC loop chooses and completes an illusory boundary that passes through all of the line corners. More precisely, a multiple-scale CC loop reaction chooses and completes several illusory boundaries, one for each spatial scale at which end cuts due to the two images can be binocularly fused. Thus, several copies of the CC loop exist within the BC System, one for each spatial scale.

\section{Suppression of Double Images by the First Competitive Stage}

In the Kaufman stereogram, perpendicular scenic edges that excite the two eyes at the same positions are rival- 
rous, thereby implicating the second competitive stage. In the Kulikowski stereogram, parallel scenic edges that excite the two eyes at disparate positions are rivalrous. This is the type of competition that occurs at the first competitive stage (Figures 2 and 3). The rivalry between the disparate vertical edges of the Kulikowski stereogram is initiated when the first competitive stage causes parallel, but disparate, orientations from both eyes to compete at the second competitive stage. Thus, the Kulikowski and Kaufman stereograms are mechanistically differentiated by the way in which they differentially engage the first and second competitive stages, respectively.

The suppressive action of the first competitive stage seems paradoxical when it is perceived in demonstrations of binocular rivalry. The same mechanism, however, is used to help suppress monocular information that cannot be fused into a binocularly consistent 3-D percept. Thus, the first competitive stage is one of the mechanisms whereby binocular double images, or the many possible combinations of false binocular matches (Julesz, 1971; Sperling, 1970), are suppressed before they can generate completed binocular boundary segmentations within the CC loop. In summary, the mechanisms of the first and second competitive stages begin to explain how the percept of a monocularly viewed image can be suppressed when a binocularly discordant image is viewed through the other eye.

Further consideration of the Kulikowski percept begins to shed light on other important issues: Why are fusion and rivalry alternative binocular modes? How does fusion at one spatial scale coexist with rivalry at a different spatial scale that represents the same region of visual space? Why can certain lower spatial frequency components be fused whereas certain higher spatial frequencies annot be fused at a fixed disparity? What definition of spatial scale can accommodate all of these properties?

\section{The Complex Cells of the OC Filter are Binocular: A Positionally Sharp \\ but Deformable Binocular Space}

The role of the first competitive stage in initiating suppression of binocular double images leads toward an understanding of these properties. In particular, why does the first competitive stage not cause rivalry between the disparate monocular images in all spatial scales? How can some spatial scales binocularly fuse images at the same disparity that at other spatial scales leads to binocular rivalry? In order for this to happen, disparate monocular images that are fused within a given scale must both input to the on-center of the scale's first competitive stage (Figure 6a). In contrast, disparate monocular images that are rivalrous within a given scale must compete via the off-surrounds of that scale's first competitive stage (Figure 6b).

This distinction suggests several conclusions: The output cells of the $\mathrm{OC}$ filter are themselves already binocular. Section 21 of Grossberg (1987b) identifies these cells with contour-sensitive complex cells in area 17 of the stri-

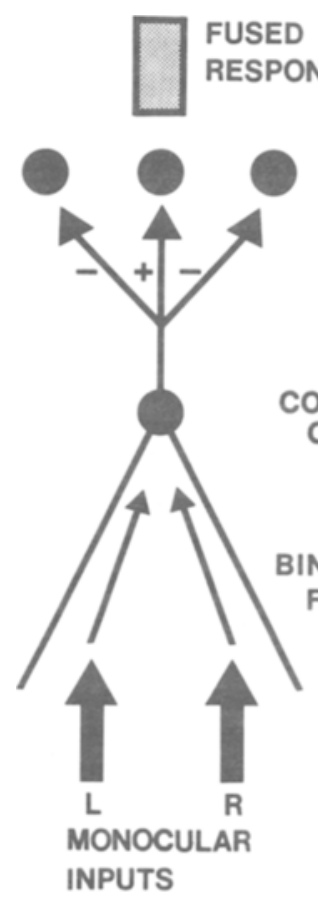

(a)
MUTUAL INHIBITION

Figure 6. Initial processing stages leading to fusion or rivalry: (a) Spatially disparate monocular images from left (L) and right (R) eyes activate a shared population of complex cells, which in turn activate a "fused" locus of cells within an on-center of the second competitive stage. (b) At a smaller spatial scale, the same monocular images may activate spatially disjoint sets of complex cells, which input to ofi-surrounds at the second competitive stage, thereby initiating rivalry between the images.

ate cortex. Complex cells are, in fact, well known to be binocular (Hubel \& Wiesel, 1962, 1968, 1970, 1977; Poggio, Motter, Squatrito, \& Trotter, 1985). Thus, the second competitive stage is binocular because its inputs from the complex cells are already binocular. Figure 6 suggests that there exist disparities at which low spatial frequency scales can fuse monocular image elements (Figure 6a) but high spatial frequency scales cannot (Figure 6b). As a result, there exist disparities at which complex cells tuned to low spatial frequencies can excite the on-center of the corresponding second competitive stage (Figure 6a) but complex cells tuned to high spatial frequencies generate mutually inhibitory signals to the second competitive stage (Figure 6b). I return in Section 24 to the question of how this relationship between spatial frequency, disparity, fusion, and rivalry can be mechanistically realized. For the moment, Figure 6 provides a pictorial way to understand how some spatial scales can fuse images that other spatial scales cannot, and how CC loop mechanisms can inhibit binocularly discordant boundary signals within those scales that are incapable of fusion.

Figure 6 also hints at how another important property of binocular space is realized. In order to fuse pairs of monocular images that are spatially disparate, the binocu- 
lar space must be deformable: Two images must be deformed into one image, much as in the phenomenon of displacement, or allelotropia (von TschermakSeysenegg, 1952; Werner, 1937). In this phenomenon, when a pattern $A B C$ is viewed through one eye and a pattern $A B C$ is viewed through the other eye, the letter $B$ can be seen in depth at a position halfway between $A$ and $C$. Although deformability implies that there exists a certain degree of positional uncertainty at early stages of binocular processing, the final binocular percept is often positionally sharp. In Section 24 , I describe mechanisms whereby the ostensibly conflicting properties of deformability and positional sharpness are realized within a binocular space that is built up by using a multiple-scale, binocular version of OC filter and CC loop mechanisms.

\section{Binocular Switching, Boundary Frames, and Negative Afterimages}

Yet another test of the hypothesis linking binocular rivalry to CC loop mechanisms concerns the issue of why rivalrous percepts switch suddenly, and approximately periodically, between representations of left image and right image. I trace this type of switching to the existence of habituating chemical transmitter substances which are hypothesized to multiply, or gate, signals within the CC loop. I assume that these transmitters occur along the feedback pathways from the cooperative stage to the dipole field (Figure 3). Thus, the CC loop contains a specialized type of gated dipole field (Grossberg, 1976b, 1980, 1983a).

Spontaneous switching behavior has previously been shown to be a property of a gated dipole field in response to image pairs that create approximately balanced, but competitive, input patterns (Grossberg, 1976b, 1980). Periodic switching occurs because the habituating transmitters within a winning channel weaken the competitive advantage of that channel by causing a decrease in the size of its positive feedback signals. The inhibited channel can then win the competition because its transmitters are able to accumulate while it is being inhibited. Then the cycle of rivalry repeats itself, leading to cyclic recovery and habituation of transmitter gates as a given channel periodically loses and wins the competition.

In order to explain the binocular rivalry that occurs in response to the Kulikowski stereogram, the positive feedback must influence the stage at which the competing inputs are registered. This stage is the second competitive stage (Figure 3). Thus, the CC loop, which was derived from constraints about monocular boundary completion and segmentation, possesses feedback pathways that are correctly placed to explain binocular rivalry.

In Grossberg (1980), the habituating gates within a gated dipole field were shown to be an important mechanism for dynamically stabilizing the adaptive tuning of cortical filters against persistent recoding by irrelevant cues (also see Section 23 below). In the special case of the gated dipole field within the CC loop, another useful function can be noted. Consider a moving vertical scenic edge that activates, then passes over, a vertically oriented cell of the CC loop dipole field. The offset of the vertically oriented input to these cells can trigger a momentary activation of horizontally oriented cells corresponding to the same position. This opponent reaction, or antagonistic rebound, helps to prevent a blurring of the vertical edge as it moves across the BC System by rebalancing the orientational competition in readiness for the next oriented input. The importance of registering moving edges as successive boundary frames, rather than as a blurred optical flow, has been emphasized in the classical studies of Johannsen $(1973,1975,1978)$. Such an antagonistic rebound within a gated dipole field is mediated by its habituating transmitter gates. The theory hereby traces the periodic nature of binocular rivalry, as well as such other metastable percepts as the Necker cube (Grossberg, 1980), to mechanisms for dynamically stabilizing cortical learning and for dynamically rebalancing cortical competitive mechanisms.

When a BC is kept active long enough for its transmitter gates to significantly habituate, a larger and more sustained antagonistic rebound is caused by offset of the inducing image, thereby causing a negative afterimage. Parametric properties of the negative afterimages due to dipole field rebounds have been compared with monocular and binocular perceptual data in Grossberg (1976b, 1980). A more detailed understanding of oriented afterimages can be derived from properties of the feedback interaction that occurs between the second competitive stage and the cooperative stage of the CC loop (Figure 7). MacKay (1957) has reported that when an image with radial symmetry (Figure $7 \mathrm{a}$ ) is inspected for a long time, offset of the pattern can generate a negative afterimage with radial symmetry (Figure 7c). This is explained by CC loop mechanisms as follows. Each position and orientation that is activated by the image habituates its transmitter gates within the CC loop. Since the gated dipole field is tonically active (Grossberg, 1987b, Section 19), offset of the image triggers antagonistic rebounds within the gated dipole field. In particular, because orientational competition occurs within the second competitive stage, offset of an orientation at a given position can activate the perpendicular orientation at that position via such an antagonistic rebound (Figure $7 \mathrm{~b}$ ). These perpendicular orientations can then collinearly cooperate with the cooperative stage to complete bands of circular negative afterimages (Figure 7c).

The above discussion suggests how circular boundary segmentations can be induced by offset of a radially organized scenic image. It does not, however, explain how we see these circular bands, since all boundaries are invisible within the BC System (Grossberg, 1987b, Section 22). The FC System also contains gated dipole fields, which are specialized as double-opponent networks of color-sensitive cells. These double-opponent color fields can also experience antagonistic rebounds when a sustained inducing image is shut off. Below I explain how these two types of gated dipole fields interact to generate 


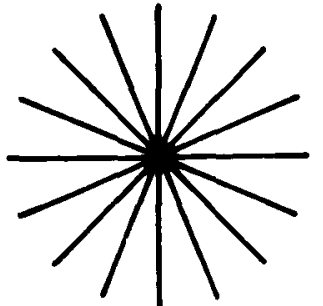

(a)

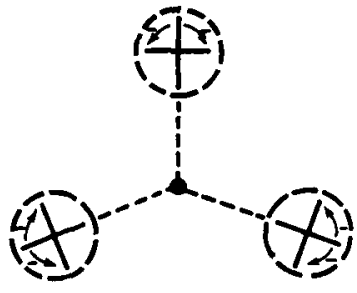

(b)

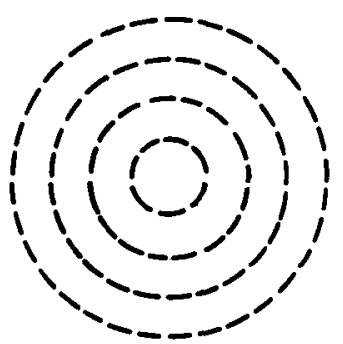

(c)

Figure 7. Negative aftereffects in orientation: (a) A pattern with radial symmetry is inspected for a long time. (b) Orientational competition occurs at each position of the second competitive stage. (c) Ofiset of the radial pattern elicits antagonistic rebounds that activate the perpendicular orientations, which thereupon collinearly cooperate with bipole cells of the $\mathrm{CC}$ loop to form circular emergent boundaries.

visible percepts of form-and-color-in-depth, including visible percepts of negative afterimages. In order to reach this insight, I momentarily turn away from the delightful phenomena of binocular rivalry to follow a conceptual path whereby a number of additional mechanistic insights about 3-D perception can efficiently be motivated and derived. Several simple gedanken experiments (Grossberg, $1983 b$ ) guide the choice of this path.

\section{Scenic Edges and Fixations: \\ Binocular Boundary Matches \\ Support Depth Percepts}

Suppose that an observer attempts to fixate a perceptually uniform rectangle hovering in space in front of a discriminable but perceptually uniform background. How does the observer know where to fixate the rectangle? Suppose, for example, that each of the observer's eyes independently fixates a different point of the rectangle's interior. Both eyes will register identical input patterns near their fixation points due to the rectangle's uniformity. The monocular visual patterns near the fixation points match no matter how large a disparity exists between the chosen fixation points, just as long as both fixation points fall well within the rectangle.

This simple example shows that binocular visual matching between spatially homogeneous regions contains no information about where the eyes are pointed, since all binocular matches between homogeneous regions are equally good no matter where the eyes are pointed. Many false matches will be generated if spatially homogeneous visual data can influence percepts of depth.

The only binocular visual matches that stand out above the baseline of multiple false homogeneous matches are those that correlate spatially nonuniform, or "edge-like," data to the two eyes. Once one has distinguished BCs from FCs, it becomes clear that the binocular matches in question occur within the BC System.

It is well accepted that the disparities of contours within the two monocular images registered by the eyes provide information about the depths of perceived objects. The above argument also shows, however, that disparity information is insufficient. Even if one computes disparities from binocular matches of BCs, the disparities will depend upon the fixation points of the two eyes. Thus, disparity information combines with information about the vergence angle of the two eyes to determine where an object is in space (Foley, 1980; Sperling, 1970). The present article will not discuss the role of vergence in establishing absolute percepts of depth. Instead, mechanisms leading to relative percepts of depth will be analyzed.

Once one agrees that binocular matches within the BC System help to generate such relative percepts of depth, it becomes vital to explain how such a binocular match is computed. In particular, data on binocular rivalry such as we have just considered lead us to raise the question: How can a binocular mismatch within the BC System suppress the visibility of monocular image data whose boundaries lose the binocular competition? This question leads to a related question: From a functional viewpoint, why should a binocular mismatch suppress the percept of the monocular image that loses the binocular competition? The second gedanken experiment helps to answer this question.

\section{Multiple Spatial Scales: Distinguishing Size from Depth}

The second gedanken experiment suggests the need for multiple spatial scales, such that only those scales capable of supporting a match can be allowed to generate a visible percept. This gedanken experiment can also be phrased in terms of the fixation process.

As a rigid object approaches an observer, the binocular disparities between its nonfixated features increase proportionally. In order to maintain the fixation process and to achieve a percept of object permanence, mechanisms capable of correlating these progressively larger disparities are needed. Other things being equal, the largest disparities will lie at the most peripheral points on the retina. The cortical magnification factor, whereby cortical regions of fixed size process larger retinal regions as a function of retinal eccentricity, is one mechanism whereby this may be accomplished (Hubel \& Wiesel, 1977; E. L. Schwartz, 1980).

It is not sufficient, however, for a single spatial scale to exist at each retinal position, such that scale size increases with retinal eccentricity. This is because objects 
of different size can approach an observer. Without multiple scales that correspond to each retinal position and can correlate information about object size with information about object depth, the observer can confuse object size with object depth. In particular, objects of different sizes can generate the same monocular retinal image if they lie at different distances from an observer, with larger objects further away. On the other hand, the boundary disparities of their paired retinal images carry information about their depth, since objects at different depths generate different binocular disparities. Multiple spatial scales corresponding to each retinal position can carry out these multiple disparity computations and disambiguate image size from image depth.

This gedanken experiment suggests the functional utility of suppressing monocular image data which binocularly mismatched boundaries. Each monocular image can excite more spatial scales corresponding to each retinal position than can binocularly match. Only the monocular boundaries that are capable of being binocularly matched provide correct information concerning form-in-depth. Consequently, the mismatched boundaries must be prevented from contributing to percepts of 3-D form.

The gedanken experiment also clarifies the utility of allowing lower spatial frequencies to match and be fused at disparities such that higher spatial frequencies are suppressed or rivalrous (Section 6). As an object approaches an observer, the sizes of its monocular retinal images and their binocular disparities increase together. Other things being equal, larger spatial scales (lower spatial frequencies) should therefore be able to binocularly match pairs of images with larger disparities than can be fused by smaller spatial scales (higher spatial frequencies).

\section{Monocular Self-Matches: \\ Gradient Depth and Motion Depth}

When an observer closes one eye, vivid perception is still possible. Moreover, monocular percepts can retain a significant impression of depth. Thus, a binocular match within the BC System is not necessary to generate a conscious percept, let alone a depthful percept. What is needed is the absence of a binocular mismatch. Because the visual world can vividly be perceived through a single eye, certain BC system cells must be capable of being monocularly activated. I call such activation a monocular self-match to distinguish it from a binocular match (Grossberg, 1983b).

More monocular self-matches can occur in the absence of binocular mismatches than can under binocular viewing conditions. This property helps to explain why, when objects are viewed under reduction conditions (one eye looks through a small aperture in dim light), depth percepts can be ambiguous (Gogel, 1956, 1965, 1970). On the other hand, the existence of more monocular selfmatches raises the question of why depth is ever perceived under monocular viewing conditions. One factor is the correlation between scale size and fusible disparities that was discussed in Sections 6 and 11. Larger scales can, other things being equal, preferentially respond to larger image elements. Under binocular viewing conditions, larger images are often closer and generate larger disparities. Due to the preferential response of larger scales to large image elements, a monocularly viewed image that contains spatial gradients (Figure 8) can be parsed among multiple spatial scales in a manner similar to its parsing during binocular viewing conditions. Gibson (1950) is notable among classical perceptual theorists for his many illustrations of how spatial gradients can influence depth perception.

A monocularly viewed moving object can activate a succession of monocular self-matches that are capable of matching or mismatching previous self-matches before they can be reset. Temporally staggered pairs of monocular self-matches can thereby generate matches or mismatches across the multiple spatial scales, which are akin to the matches due to binocular image disparities. Due to the correlation between scale size and maximal fusible disparity, faster motions can preferentially activate larger spatial scales. Thus, moving objects can cause preferential activation of some spatial scales over others, thereby enhancing a percept of depth.

The present theory suggests, more generally, that any visual operations that cause equivalent activations of multiple-scale BC System computations tend to generate equivalent depth percepts, whether they are due to monocular spatial gradients, monocular motion cues, binocular disparities, or top-down "cognitive contours." This tenet of the theory is called the principle of scale equivalence. Scale equivalence does not deny the possibility, outlined in Section 32 of Grossberg (1987b), that

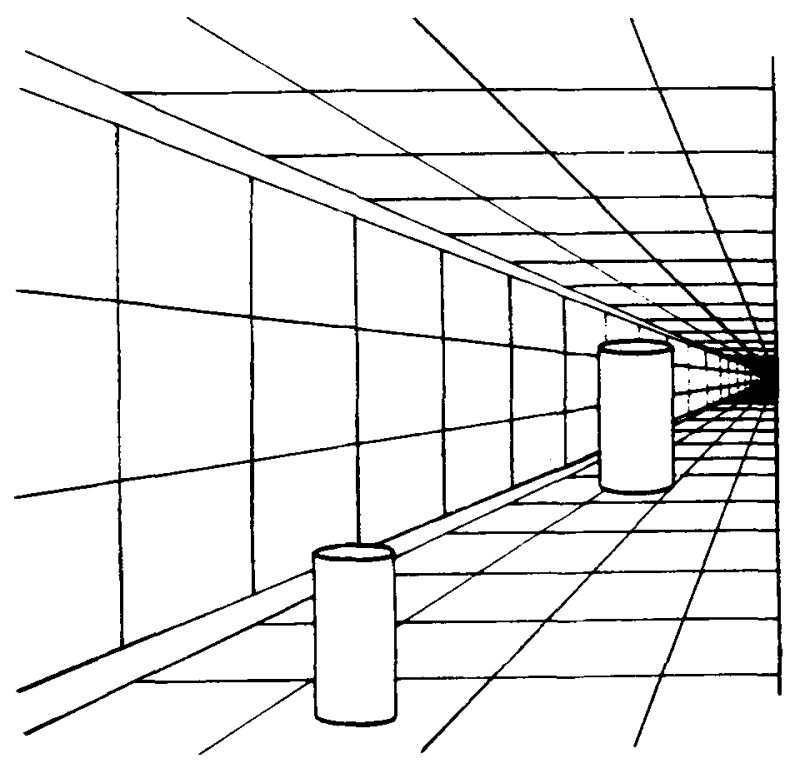

Figure 8. The corridor illusion: Due to the spatial gradients within the figure, the upper cylinder looks larger than the lower cylinder, although both cylinders are the same size. From Sight and Mind: An Introduction to Visual Perception by L. Kaufman, 1974, New York: Oxford University Press. Copyright 1974 by Oxford University Press. Reprinted by permission. 
separate BC subsystems process distinct aspects of static form and form-from-motion (Beverley \& Regan, 1979; Regan \& Beverley, 1979; Regan \& Cynader, 1982) before integrating these computations into a final 3-D percept. On the other hand, scale equivalence emphasizes that the BC System of primary interest in this article can generate depthful segmentations in response to multiple sources of monocular and binocular visual information, rather than being restricted to a single informational source-such as binocular disparity or monocular gradients-as is the case in many models of image processing.

\section{Continuous Modulation of \\ Multiple Scale Activity}

As an observer moves within a scene, the scenic forms and their depths seem to change continuously. On the other hand, at most, a finite number of spatial scales can exist in the brain. In many contemporary models of binocular depth perception, depth jumps discretely between a few values as an observer moves about. Moreover, these binocular models do not explain how the computation of disparity values leads to a percept of formand-color-in-depth. Grossberg (1983b) summarizes and analyzes a number of these models.

The present theory suggests that multiple spatial scales exist within the BC System and that these scales can be simultaneously activated by a monocularly or binocularly viewed scene, albeit by different amounts corresponding to different scenic positions. As an observer moves about a scene, the relative and absolute degree to which each of these multiple boundary scales is activated changes. These changes in the energy balance across multiple boundary scales alters the ability of the corresponding FC signals to generate a visible percept. I suggest that multiple FC syncytia exist corresponding to the multiple BC System spatial scales, each syncytium capable of contributing to a visible percept, but to different degrees. In the limiting case wherein no BC signal within a given spatial scale excites the corresponding FC syncytium, that FC syncytium cannot contribute to a visible percept.

This type of multiple-scale concept supports the strong kernel of truth that exists within the Fourier theory of spatial perception (Graham, 1981; Graham \& Nachmias, 1971), but also replaces the Fourier theory by one with a greater explanatory range.

\section{To Have Your Edge and Fill-In Too: The Julesz 5\% Solution}

The type of interactions that occur between the BC System and the FC System to generate a depthful percept can be motivated in several ways: The suppression of spatially uniform input patterns to extract informative signals for binocular matching (Section 10) follows from the contrastsensitivity of the BC System. It also illustrates that reduction of one type of informational uncertainty can cause a new type of informational uncertainty. For example, in response to monocular viewing of a perceptually uni- form rectangle hovering in space above a perceptually uniform background, the BC System generates a rectangular boundary and suppresses the uniform parts of both figure and ground. Why, then, do we not perceive a world of discrete boundaries, or at best a world of cartoon-like boundary segmentations? How do we perceive continuous forms?

As mentioned above, the theory suggests that boundary segmentations regulate diffusive filling-in events within multiple-cell syncytia of the FC System, such that a different syncytium corresponds to each spatial scale of the BC System. Indeed, a spatial scale of the BC System is defined to be the set of BC System receptive field sizes whose CC loop output pathways project to a single syncytium of the FC System. I show below how the locations where unambiguous depth computations can be performed, such as at the fused binocular boundaries of the BC System, regulate the multiple-scale filling-in reaction within the FC System. The selectivity of this fillingin reaction across spatial scales enables ambiguous regions, such as the interior of a homogeneous rectangle, to inherit the relative depth values computed by the fused binocular boundaries.

A beautiful example of the role of multiple-scale fillingin mechanisms in depth perception is provided by what I like to call the Julesz 5\% solution (Julesz, 1971, p. 336). This is a stereogram whose left "figure" and "ground" are both constructed from a $5 \%$ density of randomly placed black dots on white paper. In the right picture, the "figure" of dots is shifted, as a whole, with respect to its position in the left picture. As usual, the background dots in both pictures have identical positions, except for positions that are covered or created by shifting the "figure." When viewed through a stereoscope, the whole figure, including the entire $95 \%$ of white background between its dots, appears to hover as a planar surface above a planar ground.

How does the white region of the "figure" inherit the depth arising from the binocular disparities of the meagerly distributed black dots within the "figure'"? How does the white region of the "ground" inherit the depth due to binocular matching of its meagerly distributed dots? What mechanism organizes the locally ambiguous white patches that dominate $95 \%$ of the pictures into two distinct planar regions in depth?

This is not simply a matter of computing different disparity values for the two white regions, as many models have suggested (Dev, 1975; Marr \& Poggio, 1976, 1979; Sperling, 1970). Instead, the entire featural landscape of black dots on white background is split into two planar regions. An adequate explanation must show how all the filled-in featural qualities, such as black and white, can inherit the depth values computed by a sparse BC system segmentation that is itself indifferent to figural qualities because it is generated by broadband boundary detectors that are insensitive to direction-of-contrast (Grossberg, 1987b, Section 23). 


\section{Surface Curvature and Multiple Syncytial} Scales: The 21/2-D Sketch Does Not Exist

That interactions between disparity-sensitive BC System segmentations and FC System filling-in events are needed to generate a completed 3-D percept can also be seen through the following examples. When both eyes focus on a single point within a patterned planar surface viewed in depth, the fixation point is a point of zero disparity. Points of the surface that are increasingly far from the fixation point have increasingly large binocular disparities. Why does such a plane not recede toward optical infinity at the fixation point and curve toward the observer at the periphery of the visual field? Why does the plane not become distorted in a new way every time our eyes fixate on a different point within its surface? If the relative sizes of boundary disparities control relative depth percepts, then how do we ever perceive planar surfaces? How do we even perceive rigid surfaces?

The severity of this problem is further indicated by the fact that perceived depth does, under certain circumstances, depend upon the choice of fixation point. Staring at one point in a Julesz stereogram can result in a gradual loss of depth (Kaufman, 1974). Also, in a stereogram composed of three vertical lines to the left eye and just the two outmost lines to the right eye, the depth of the middle line depends upon whether the left line or the right line is fixated (Kaufman, 1974). If depth can depend on the fixation point when discrete lines are viewed, then why do observers not perceive planar surfaces as being highly curved? What is the crucial difference between the way we perceive the depths of curves and of surfaces?

These examples raise the fundamental issue of how an observer knows that a planar surface is being viewed, not just whether the observer can estimate the depths of some parts of the surface. As noted in Section 10, when a homogeneous planar surface is being viewed, it is not possible to compute any unambiguous disparity computation within the interior of the plane. Determining that such a surface is planar thus cannot just be a matter of showing that the same disparity can be computed at all interior points of the surface. In fact, the BC System suppresses the interior of the plane in order to successfully match its boundaries.

The present theory suggests that when a particular BC scale is strongly activated by a given scene, this $\mathrm{BC}$ activation can trigger a strong filling-in reaction within the corresponding syncytium of the FC System. By definition, featural filling-in within a syncytium is restricted to the spatial scale in which this syncytium resides. I suggest that a surface percept will appear flat if it is generated by a pair of bounding BCs within a single spatial scale, because featural filling-in is restricted to the single syncytial scale that these BCs activate. A surface will appear curved if a multiple-scale BC reaction causes the distribution of filled-in featural activity to be "curved" among several syncytial scales as perceptual space is traversed. Several scales can be coactivated at each perceptual location. The distribution across space of which scales are activated imparts an impression of relative depth.

This explanation of perceived surface flatness and curvature suggests that $\mathrm{FC}$ signals corresponding to a fixed retinal position send inputs to the filling-in syncytia of all the multiple spatial scales. Only those scales that also receive $\mathrm{BC}$ signals can, however, convert these FC signals into visible percepts. One of the fundamental tasks of the present binocular theory is to explain how such an interaction between BC signals and FC signals can convert some, but not all, of the FC signals into filled-in percepts.

The above considerations suggest that the hypothesis that a 21/2-D sketch exists, distinct from a full 3-D representation, is incorrect (Marr \& Nishihara, 1978). Such as $21 / 2-D$ sketch is an "orientation and depth map of the visible surfaces around a viewer" (Marr \& Poggio, 1979, p. 306). In contrast, the above considerations suggest that "ambiguous" regions of a scene, whose positions do not possess their own BCs, derive a relative depth value from the energy balance of their filled-in featural activities across all the spatial scales at that position. In other words, a depth map is completed by the multiple-scale featural filling-in process that generates a full 3-D representation of form-and-color-in-depth. Even in many filled-in 3-D representations there exist positions with indeterminate orientations because the featural fillingin process is unoriented, unlike the boundary completion process.

Although multiple-scale filling-in events within the FC System play an important role in the present theory toward imparting a relative depth value to ambiguous positions that do not possess their own binocular BCs, these events are surely not the only mechanisms used in depth perception. As noted in Section 10, vergence angle is one of perhaps several additional factors that contribute to an absolute depth percept. In addition, the mechanisms that generate a percept of 3-D form need not be identical with the mechanisms used to reach objects in space, just as the mechanisms that generate a percept of 3-D form are not identical with the mechanisms which govern the recognition of objects (Grossberg, 1987b, Section 4).

\section{Emmert's Law and Fechner's Paradox}

Even without a detailed mechanistic analysis, many paradoxical percepts are clarified by these concepts. The classical Emmert's law, for example, is consistent with the theory. Emmert (1881) showed that a monocular afterimage seems to be located on any surface that a subject binocularly fixates while the afterimage is active. Moreover, the perceived size of the afterimage increases as the perceived distance of the surface increases. The present theory suggests that binocular viewing selectively activates certain BC System spatial scales more than others, whereas the monocular afterimage may excite all scales more equally. The energetic loading of certain BC System scales selectively activates the corresponding syncytia, and thereby shifts the apparent depth of the af- 
terimage to those syncytia in which featural filling-in can most strongly occur.

The hypothesis that scale-specific BCs can selectively trigger featural filling-in reactions requires further development to explain how the $\mathrm{FC}$ signals from the two eyes contribute to the final binocular percept. One of the virtues of the FIRE process was its ability to quantitatively simulate paradoxical properties of binocular brightness percepts (Cohen \& Grossberg, 1984a), such as Fechner's paradox, binocular brightness summation, binocular brightness averaging, and aspects of binocular rivalry (Blake, Sloane, \& Fox, 1981; Cogan, 1982; Cogan, Silverman, \& Sekuler, 1982; Curtis \& Rule, 1980; Legge \& Rubin, 1981; Levelt, 1965). Fechner's paradox illustrates the type of FC interactions that must be developed within an adequate theory of binocular form perception. In its simplest version, Fechner's paradox notes that the world does not look half as bright when one eye is closed, despite the fact that half as much light activates the brain. In fact, suppose that a scene is viewed through both eyes but that one eye sees it through a neutral density filter (Hering, 1964). When the filtered eye is entirely occluded, the scene looks brighter and more vivid despite the fact that less total light reaches the two eyes. The explanation below of how BCs trigger featural filling-in explains all the binocular phenomena that the FIRE theory could explain, and clarifies the properties of the FIRE theory that led to these successes.

\section{Filling-In Generators and Filling-In Barriers: Blobs, Stripes, and Reciprocal \\ Striate-Prestriate Connections}

I can now outline a theory of how binocular interactions occur within the BC System and the FC System, and how the totality of the interactions within these systems leads to a representation that joins depth, brightness, color, and form information together within the model network that I compare to prestriate area V4. This model is consistent with recent experimental evidence that "many V4 cells exhibit length, width, orientation, direction of motion and spatial frequency selectivity. In the spectral domain, many V4 cells are also tuned to wavelength. Thus, V4 is not specialized to analyze one particular attribute of a visual stimulus; rather V4 appears to process both spatial and spectral information in parallel" (Desimone, Schein, Moran, \& Ungerleider, 1985, p. 441).

Such a multiple representation was also suggested within the FIRE theory (Cohen \& Grossberg, 1984a, 1984b; Grossberg, 1983b). The present theory agrees with the assumption of the FIRE theory that the control of featural filling-in requires multiple processing stages. The present theory makes a break with the FIRE theory by showing how these stages can all use a diffusive mechanism of featural filling-in, rather than diffusive filling-in for monocular interactions and FIRE filling-in for binocular interactions.

In both the FIRE theory and the present work, it is necessary to distinguish between filling-in generators
(FIGs) and filling-in barriers (FIBs). First I will sketch the functional ideas that necessitate this distinction and draw some general conclusions. Then I will analyze each of the mechanisms in greater detail.

Figure 1 summarizes the main properties of FIGs and FIBs. In Figure 1, boxes with vertical lines stand for stages of BC processing. Boxes with three pairs of circles stand for stages of FC processing. The lines symbolize the orientational tuning within the BC System. The pairs of circles symbolize the organization of the FC System into opponent and double-opponent cells (red-green, blue-yellow, white-black). In Figure 1, oriented monocular cells from the left eye $\left(B C S_{L}\right)$ and the right eye $\left(B C S_{R}\right)$ interact along the pathways labeled 1 to activate oriented binocular BC System cells. This interaction requires the processing stages that are schematized in Figures 3 and 6. For the moment, it suffices to say that this interaction takes place, in parallel, within multiple spatial scales, and that it generates oriented binocular cells, some of which are precisely tuned to binocular disparity. The outputs from this binocular stage generate both FIGs and FIBs. Both types of signals are assumed to operate using the same mechanism. Their different functional effects are due to their different locations within the network as a whole. FIG signals are top-down signals to a prior level of processing. Their targets are cells within the FC System which process monocular FC signals. FIB signals do not project to a prior level of processing. Their targets are cells within the FC System that process binocular FC signals.

Figure 9 schematizes the effect of a FIG signal. Contoursensitive FC System inputs that discount the illuminant are segregated within separate double-opponent channels (R-G, B-Y, W-B). These double-opponent channels are replicated so that FIG signals from each spatial scale of the BC System can interact with its own complete set of double-opponent cells and their corresponding syncytia. In Figure 9a, such an FC System input activates its target cell. This activation spreads laterally, or diffusively fills-in, from cell to cell. As in Section 24 of Grossberg (1987b), such a filling-in reaction is assumed to be accomplished by electrotonic interactions across gap junctions. Also as in Grossberg (1987b), I continue to call such a filling-in reaction a syncytial interaction. The network as a whole is called a monocular syncytium. Each FC input is distributed topographically to all the syncytia of the FC System, such that one complete set of doubleopponent syncytia corresponds to each spatial scale of the BC System. Each FIG input from the BC System is distributed topographically to all the double-opponent syncytia of its spatial scale. In all, an FC input is broadcast in a wavelength-selective and positionally selective way to the syncytia of all spatial scales, whereas a FIG input is broadcast in a scale-selective and positionally selective way across syncytia that code different wavelengths (Figure 9b).

In the absence of BC System signals, the lateral syncytial interactions elicit a spatial pattern of activation such 


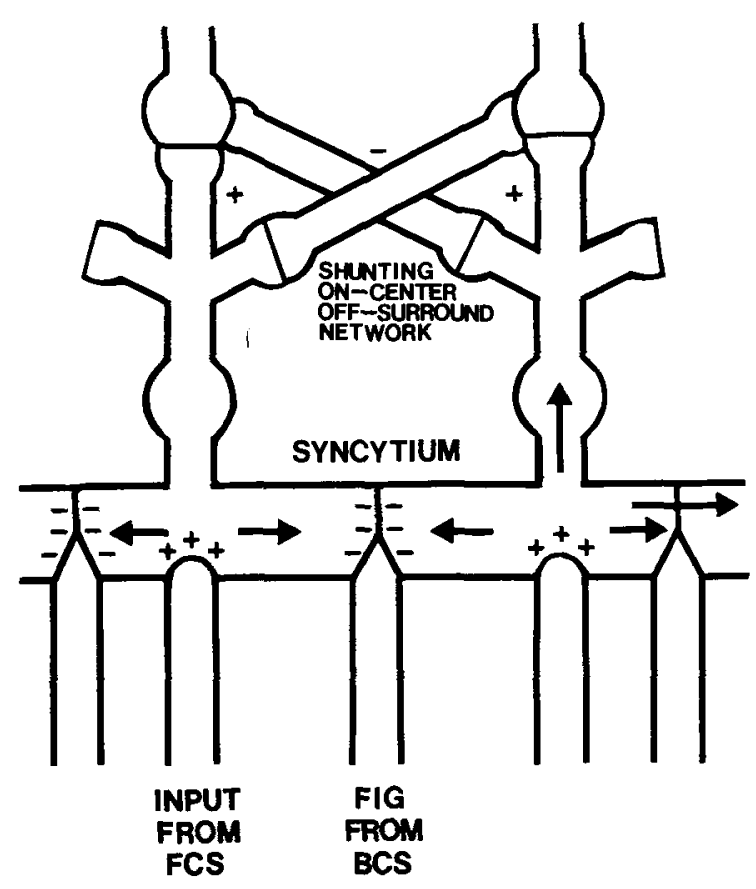

(a)

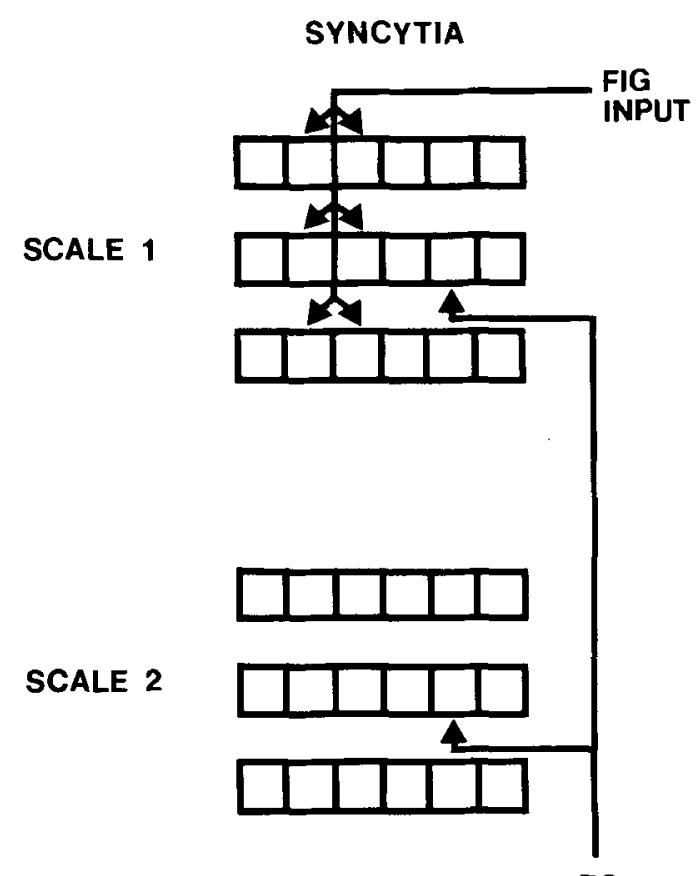

(b)
FC INPUT
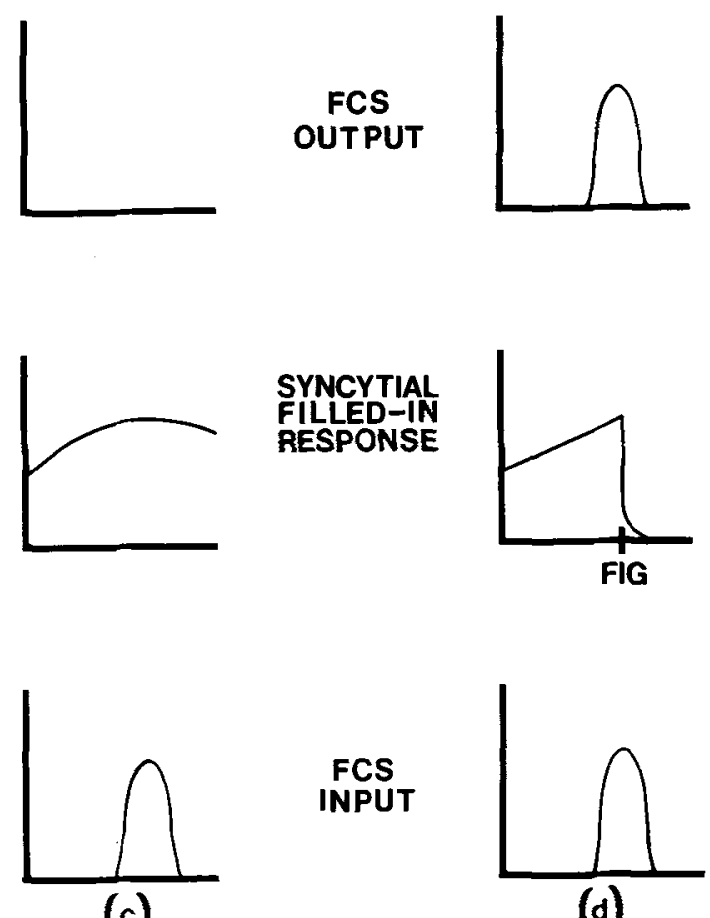

(c)

(d)

Figure 9. How filling-in generators (FIGs) control FC output signals: (a) FC inputs excite the monocular syncytium, which carries activation electrotonically to neighboring syncytial cells, except at cell membranes that receive FIG inputs. Syncytial cells activate a shumting on-center off-surround network that is sensitive to spatial discontinuities in the filled-in syncytial activity profile. Thus, if no FIG input prevents lateral spread of the FC input, then no FC output signal occurs, as in (c). (b) Each FC input is broadcast in a wavelengthselective and positionally selective way to the syncytia of all spatial scales. Fach FIG input is broadcast in a scale-selective and positionally selective way across syncytia that code different wavelengths. In (d), an FC output signal is generated because a FIG input causes a spatial discontinuity in the filled-in activity profile. 
that nearby cells have similar activity levels (Figure 9c). The syncytial cells output through a spatially short-range, shunting on-center off-surround network (Grossberg, 1983b). Such a network is sensitive to spatial discontinuities, or edges, in the activity pattern. Due to the smoothing action of the lateral interactions, the shunting on-center off-surround network does not allow any output signals to be generated.

Thus, in the absence of FIG signals from the BC System, the FC inputs to the monocular syncytium cannot generate contour-sensitive output signals from the monocular syncytium. Since only the FC System syncytia that also receive FIG signals can elicit output signals, one can begin to see how the FC System can generate scaleselective visible representations despite the fact that all of its syncytia receive the same FC inputs at an early processing stage. I now explain how FIG signals enable some of the contour-sensitive FC inputs to generate contour-sensitive FC outputs to the next processing stage.

When an FC input occurs at the same time as a contiguous FIG signal from the BC System, the FIG signal acts, as described in Figure 17 of Grossberg (1987b), to prevent the lateral spread of activation across its target cells (Figure 9d). A spatial discontinuity is hereby created in the spatial pattern of activation. The shunting on-center off-surround network senses this spatial discontinuity, and generates an output signal from the locations where it occurs. Due to properties of such shunting networks, the size of this output signal is sensitive to both the relative and absolute sizes of the spatial discontinuity as compared with the level of background activity (Grossberg, 1983b, 1987a). Thus, these FC output signals have useful properties for building up ecologically useful color percepts (Cohen \& Grossberg, 1984a).

In summary, a FIG signal from the BC System allows an FC input to generate an output from the monocular syncytium by creating a spatial discontinuity in the spatial patterning of activity that is caused by the input. No FC output can occur, even in response to a large FC input, unless such a FIG, or binocular boundary signal, is received from the BC System. This property will be crucial in my explanation of how we typically see a globally self-consistent binocular percept uncontaminated by the existence of many binocularly discordant monocular inputs.

The type of featural filling-in which occurs within the monocular syncytium can be effective even if its interaction range, or spatial bandwidth, is relatively narrow. It need only smooth the FC input over a spatial domain that is wider than the spatial bandwidth of the short-range shunting on-center off-surround network, so that the filledin activities are not processed as "edges" by this network.

Rockland and Lund (1983) and Livingstone and Hubel (1984a, 1984b) have both reported lateral interactions among the cytochrome oxydase staining blobs of area 17 . Several facts are consistent with the interpretation that the monocular syncytium may include the blobs: Many of the blob cells are monocular (Livingstone \& Hubel, 1984a).
The interblob interactions are of relatively short range. Livingstone and Hubel (1984a, p. 353) go so far as to comment that "the blob cells... are probably too short range in their spatial coverage to generate the long-range interactions that occur in color perception." If this anatomical interpretation of the monocular syncytium is correct, then two types of heretofore unreported interactions should exist: (1) prestriate-to-blob signals capable of gating shut the lateral interblob interactions; (2) post processing of blob cells such that only spatially discontinuous, or edge-like, blob activity patterns can generate output signals to the area 18 stripes to which the area 17 blobs project (Livingstone \& Hubel, 1984b). If this syncytial interaction does not occur among the blobs, then it should occur at target cells of the blobs.

\section{Binocular Matching of Color Edges and Featural Filling-In}

The output signals from the left and right monocular syncytia are labeled 2 in Figure 1. These output signals play the role of color contours in the theory. In order to generate percepts of color fields and surfaces, these color contours must also trigger a featural filling-in reaction. To this end, the color contour signals project topographically to binocular double opponent cells that are hypothesized to exist within the binocular syncytium of Figure 1. A separate complete set of double opponent binocular syncytia exists corresponding to every spatial scale of the BC System. The discussion in Section 17 showed that a binocular syncytium within the FC System receives a color contour signal only at positions that can generate a binocular boundary signal within the BC System. As in the monocular syncytium, FC inputs to the binocular syncytium trigger a lateral spreading, or diffusive filling-in, of featural activity, which spreads until it runs down its own electrotonic gradient or until it hits a cell membrane that acts as a high resistance barrier.

Signals from the BC System generate such barriers in a binocular syncytium much as they do within a monocular syncytium. Within a binocular syncytium, boundary signals from the BC System are called FIBs rather than FIGs, even though their local mechanisms are assumed to be identical. How is a FIB signal generated within the binocular syncytium at every position that may receive an FC output from a monocular syncytium? The answer suggested in Figure 1 is that both FIGs and FIBs are elicited by the same BC System segmentation. Since FC outputs are regulated by the positions of FIG signals to the monocular syncytia, FIB signals will exist at all positions of the binocular syncytia that may be expected to receive these $\mathrm{FC}$ outputs.

Pairs of monocular FC signals input topographically to the binocular syncytium that corresponds to their own color and spatial scale. Because these inputs are processed by a network of double opponent cells, chromatically similar inputs that are spatially disparate may inhibit one another, whereas inputs that are sufficiently well matched spatially can elicit a synergetic reaction. I assume that the 
double-opponent network is a specialized gated dipole field (Grossberg, 1976b, 1980, 1983a). In such a network, shunting on-center off-surround interactions within a color channel coexist with shunting off-center on-surround interactions within the opponent color channel, and the opponent color channels compete with each other at each position. (Also see Section 26.) Cohen and Grossberg (1984a) have demonstrated that monocular and binocular featural inputs to such a shunting on-center offsurround network generate activity patterns whose properties are strikingly similar to data about monocular and binocular brightness perception. In the Cohen and Grossberg (1984a) article, a FIRE process fills-in between the color contours. The FIRE process acts to binocularly summate and fill-in activities within perceptive regions between binocularly matched boundaries. Since a FIRE process is defined by a shunting on-center off-surround network, its monocular and binocular activity levels are the same as those generated by the shunting on-center offsurround network that defines a color channel. In the present theory, filling-in of these values by FIRE is replaced by diffusive filling-in of these values under the enabling influences of FIGs and FIBs. Since the filled-in values generated by a FIRE and those generated by diffusive filling-in both average the values computed by the shunting network, the Cohen and Grossberg (1984a) computer simulations of monocular and binocular brightness data are also valid in the present theory. However, the present theory uses only a single mechanism of featural filling-in, namely electrotonic or diffusive filling-in, for both monocular and binocular featural interactions.

\section{A Representation of Form-and-Color- in-Depth}

Even in the absence of further details, the perceptual representations that arise within the binocular syncytium can be seen to be sensitive to such factors as orientation, spatial frequency, depth, form, and color, as Desimone, Schein, Moran, and Ungerleider (1985) and Zeki (1983a, 1983b) have reported in V4. The sensitivity to orientation is due in part to the orientation-sensitive inputs from the BC System (but also see Section 29). Sensitivity to spatial frequency is due to the parsing of both BC System and FC System subnetworks into separate spatialfrequency-sensitive channels. Sensitivity to depth is due to the disparity-sensitive inputs from the BC System, as well as to the possibility that spatially disparate monocular FC System inputs can mismatch within a doubleopponent network. Sensitivity to form follows from the ability of the BC System to preattentively complete boundaries and to segment textures, as well as to receive learned and attentionally modulated boundary completion signals from the Object Recognition System, before generating FIB signals and FIG signals to the FC System (Grossberg, 1987b, Figure 2). Sensitivity to color derives from the fact that successive stages of FC System processing progress from opponent to double-opponent processing. A single cell within the binocular syncytium can thus be sensitive to all of these factors. Such a cell is also sensitive to featural filling-in across a spatial domain that is determined by the global configuration of all BC and FC signals.

Neurophysiological data have not yet disentangled the separate input and interaction pathways that can give rise to such a complicated cellular response. In particular, direct evidence is lacking for the existence of a binocular cortical cell syncytium or, for that matter, for lateral interactions, although interactions of some type are known to influence V4 cells over regions corresponding to as much as $30^{\circ}$ on the retina. Although direct evidence is, as yet, lacking, some indirect evidence has been found. The evidence of Zeki (1983b) is sufficiently important to quote at length:

It has been very difficult to determine the extent and the disposition of the critical surround.... Adding middle or short wave light... led to a response, but only after a delay of about $4 \mathrm{~s}$. The reverse sequence of stimulation also led to a response, but again with a delay. Compared to the almost instantaneous reactions of... cells in V1, this in itself suggests indirectly that the cell is responding only after integrating information from large parts of the field of view.... The actual position of the rest of the. . display... .did not make a difference to the cell's response. (pp. 775-776)

Both the long delay before the cell responded (which may be considerably shortened in an unanesthetized animal) and the cell's insensitivity to changes in the positioning of the rest of the display are consistent with the existence of a lateral filling-in reaction that averages its activations from all the color edge inputs that are contained within the surrounding boundary.

\section{Interactions Between Brightness and Depth Information}

The concept that multiple scale filling-in imparts a relative depth value to ambiguous regions implies that brightness and depth information can mutually influence one another. Grossberg (1983b) reviews classical data that are consistent with this assertion. Two types of more recent data are summarized in this section for illustrative purposes.

B. J. Schwartz and Sperling (1983) and Dosher, Sperling, and Wurst (1986) have analyzed influences of luminance differences upon both perceived depth and perceived rigidity of form. In their studies, they consider proximity luminance covariance (PLC) as a factor influencing percepts of depth and form. To manipulate PLC, the luminance of each line in a 2-D projection of an object was made to depend on the 3-D depth of that line. A larger luminance was used to signal a closer object projection. PLCs that confirm and that conflict with the 3-D depth were analyzed. The interaction of PLC with stereo information was also analyzed. Both studies concluded that PLC is a powerful factor in determining a depth percept and that, moreover, stereo and PLC information combine in a way that can be summarized by a weighted linear model. 
Egusa (1983) has studied the effects of luminance differences on perceived depth by constructing stimuli consisting of two hemifields of different colors, and asking the subject to state which appeared nearer and to judge the peceived depth between them. When both hemifields were achromatic, the perceived depth increased with increasing brightness difference. With chromatic-chromatic combinations, the perceived depth depended upon the hue combination. In terms of decreasing frequency of "nearer" judgments, the hue order was red, green, and blue.

\section{Transparency}

Transparency phenomena (Beck, Prazdny, \& Ivry, 1984; Metelli, 1974; Metelli, Da Pos, \& Cavedon, 1985; Meyer \& Senecal, 1983) provide another type of data that support the concept that multiple syncytia exist corresponding to different spatial scales, and that filling-in of FC signals within some of these syncytia but not others can generate a percept of form-and-color-in-depth. In percepts of transparency, a phenomenal scission occurs which replaces the percept of a single color at a fixed perceptual location with the simultaneous perception of two colors: the color of the object seen through the transparency and the color of the transparent layer. Within the theory, such a scission is analyzed by considering how one color elicits filling-in within a syncytium of one spatial scale, whereas the other color elicits filling-in within a syncytium of a different spatial scale. Such an analysis is possible due to the hypothesis that each FC signal is topographically broadcast to the syncytia of all spatial scales, and that the spatial distribution of BC signals among the several scales determines which of the syncytia will react to such an FC signal by triggering a fillingin reaction.

The formal rules articulated by Metelli (1974) for predicting the occurrence of transparency are similar to the conditions under which the BC system triggers neon color spreading (Redies \& Spillman, 1981; van Tuijl, 1975; van Tuijl \& de Weert, 1979) within the FC System. This relationship is perhaps best seen from Meyer and Senecal's (1983) study, which used a variant of the Kanizsa $(1976,1979)$ subjective contour configuration. Unlike Figure 5 in Grossberg (1987b), some of the Pacman figures they used to induce a rectangular subjective contour were completed into circles within the rectangle, using faintly colored wedge-shaped regions. Meyer and Senecal showed that a percept of transparency covaried with the percept of a chromatically filled-in rectangle surrounded by a strong rectangular subjective contour. In an explanation of neon color spreading (Grossberg \& Mingolla, 1985a), we maintained that the strength of such a subjective contour also regulates the strength of chromatic filling-in reactions by inhibiting boundaries that would otherwise prevent filling-in from escaping from the colored inducing wedge-shaped regions. Thus, these data are consistent with the idea that transparency is due to featural filling-in reactions across some, but not all, of the syncytia corresponding to each fixed perceptual location, and that these differential filling-in reactions are associated with the percept of a relative difference in depth.

\section{Generating Disparity-Sensitive and Spatial-Frequency-Sensitive Cells Using Positionally Ambiguous Inputs: \\ Inhibitory Interneurons and Multiplexed Complex Cells}

I now outline the theory's conception of how monocular inputs to the BC System generate the binocular and disparity-sensitive complex cells that input to the CC loop, as in Figure 6. I also suggest how these complex cells can become sensitive to orientational disparity at each position as well as to positional disparity (Shinkman \& Bruce, 1977; von der Heydt, Hänny, \& Dürsteler, 1981). Indeed, I show how individual complex cells can become sensitive to position, orientation, spatial frequency, positional disparity, and orientational disparity, yet also be broadband with respect to color in order to function as boundary detectors (De Valois, Albrecht, \& Thorell, 1982; Poggio, Motter, Squatrito, \& Trotter, 1985; Thorell, De Valois, \& Albrecht, 1984). Thus, individual complex cells multiplex many different types of perceptual information. Then spatial arrays of such complex cells input to the competitive and cooperative mechanisms of the $\mathrm{CC}$ loop, which sort out all of these different types of information into a binocularly consistent segmentation capable of suppressing many possible double images. Thus, the BC System's cells become binocular, indeed multiplexed, at an early stage to provide a processing substrate from which the CC loop's mechanisms can synthesize stereo information into its emergent segmentations. This scheme of binocular preprocessing, followed by competitive and cooperative interactions, will be seen to sharpen my explanation of the Kaufman (1974) and Kulikowski (1978) data about binocular rivalry (Sections 2-9), as well as various other data about interactions between form and color processing. In order to reach these conclusions, I briefly consider the role of developmental plasticity and intracortical inhibitory interactions in setting up the binocular BC System computation.

Figure 10a schematizes the fact that a certain amount of positional uncertainty is caused in order to form an oriented receptive field: To detect the orientation of a scenic contrast difference, the receptive field needs to collect inputs corresponding to small regions of the retinal mosaic. Thus, in response to a monocularly viewed, vertical figural edge, a spatial pattern of reactions can be generated at the earliest stage of oriented BC System processing, which I identify with the simple cells of the striate cortex (Hubel \& Wiesel, 1977). This type of positional uncertainty is functionally related to the well-known relationship between receptive field scatter and the cortical magnification factor (Daniel \& Whitteridge, 1961; Dow, Snyder, Vautin, \& Bauer, 1981; Hubel \& Wiesel, 1977). This type of positional uncertainty may also contribute to the binocular sensitivity of some striate simple 


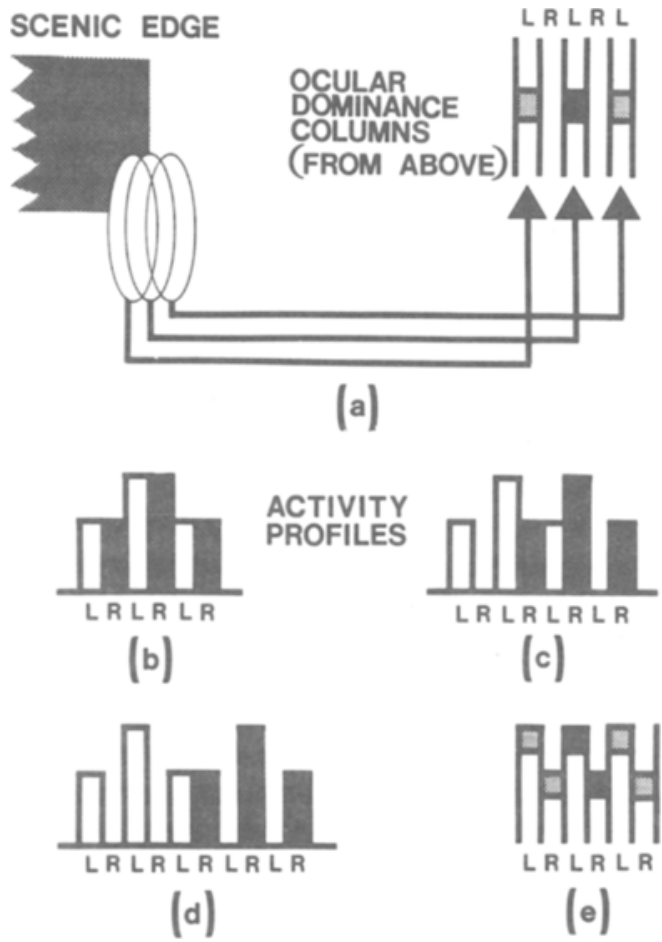

Figure 10. Translation of scenic contour information into spatial patterns of cortical activity: (a) Overlapping like-oriented receptive fields generate a spatial pattern of activity at left-monocular representations in response to a left-monocularly viewed scenic edge. The figure portrays a view from above of cortical ocular dominance columns for the left (L) and right (R) eyes, and portrays increased cell activation with darker areas. (b)-(d) Binocular inputs due to a scenic edge viewed by the two eyes at increasing positional disparities creates distinct, expanding activity patterns, across the ocular dominance columns. Here bar heights code activities. (e) Binocular viewing can cause an orientational disparity that is coded by a positional shift in the activity pattern caused by the left eye relative to that caused by the right eye. This shift is perpendicular to the shift caused by positional disparity, which separates activity patterns caused by the two eyes in a horizontal, rather than a vertical, direction.

cells (Poggio, Motter, Squatrito, \& Trotter, 1985) by contributing to the overlap of ocular dominance columns.

The positional uncertainty property raises the following basic issue. The receptive fields of simple cells enable them to respond selectively to properties of orientation and spatial frequency within a scene. These receptive fields also, however, suffer a loss of positional information, since receptive fields corresponding to several retinal positions may be activated by a single scenic position. How can subsequent cortical interactions compensate for the loss of positional information that is required to design orientationally selective receptive fields? I suggest below how the processes that restore a greater measure of positional certainty also generate binocular complex cells whose tuning curves are sensitive to positional disparity, orientational disparity, and spatial frequency.

The technical challenge met by a network capable of this task can be seen by comparing Figures 10b-10e, which show that as the positional disparity or the orientational disparity of a binocularly perceived edge varies, it elicits a different spatial pattern of activation across the network of oriented receptive fields. As the input disparity changes, the pattern of excitation can change not only its internal structure, but also its spatial scale. How can such spatial patterns be used to activate binocular cells that are sensitive to positional disparity, orientational disparity, and spatial frequency? Several models for the development of binocular tuning in cortical cells have been advanced; these include those of Bienenstock, Cooper, and Munro (1982), Grossberg (1980, 1983a), Singer $(1983,1985)$, and Willshaw and von der Malsburg (1976). None of these models has, however, dealt directly with the problem schematized in Figure 10. A solution is suggested by the use of a general neural network designcalled a masking field-which has heretofore been applied to explain data concerning visual masking (Grossberg \& Levine, 1975) and context-sensitive encoding of speech sounds and cognitive recognition codes (Cohen \& Grossberg, 1986, in press; Grossberg, 1978, 1987a). I suggest that variants of the masking field design may be used by the nervous system wherever functional properties of the following type are needed.

In a masking field model, one level, $F_{1}$, of cells sends input pathways to a second level, $F_{2}$, of cells. Level $F_{1}$ generates spatial patterns of activation that can vary both in their internal structuring and in their spatial scale, as in Figure 10. These spatial patterns of activation across $F_{1}$ generate spatially distributed inputs to $F_{2}$ (Figure 11 ). In particular, each active $F_{1}$ cell can send signals to many $F_{2}$ cells along pathways whose connection strengths decrease with the lengths of the pathways. Thus, the positional uncertainty of the responses by $F_{1}$ cells in Figure 10 is rendered even more ambiguous by the divergence of signal pathways from each cell in $F_{1}$ to $F_{2}$. Despite this

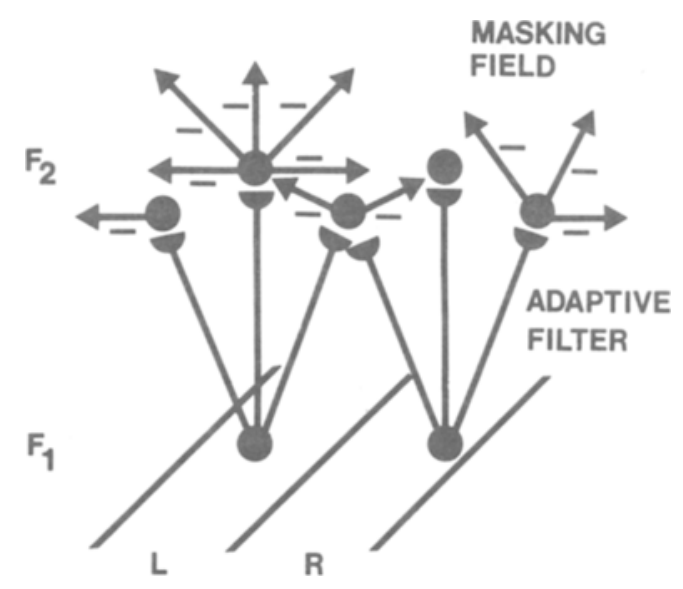

Figure 11. Output signals from oriented monocular representations ( $L$ and $R$ in level $F_{1}$ ) converge via an adaptive filter at cells that compete via a masking field (level $F_{2}$ ). Only cells whose inputs survive the masking-field competition to generate a short-termmemory activation can trigger learned changes in the abutting longterm-memory traces that are found in the $F_{1} \rightarrow F_{2}$ pathways. 
fact, level $F_{2}$ can generate spatially localized activations in response to the distributed input patterns from $F_{1}$. Moreover, these spatially localized $F_{2}$ activations represent narrowly tuned reactions to positional disparity, orientational disparity, and spatial frequency information (Grossberg \& Marshall, 1987).

These formal properties are due to three types of network interactions: First, the divergence of signals from each cell of $F_{1}$ to $F_{2}$ implies a convergence of $F_{1}$ signals at each cell of $F_{2}$ (Figure 11). Such a convergence of signals enables $F_{2}$ to filter the activation pattern across $F_{1}$.

Second, level $F_{2}$ contains a network of intrinsic inhibitory interneurons. The design of this network enables it to suppress, or mask, the activations of all $F_{2}$ cells except those which best represent the total activation pattern across $F_{1}$. In particular, many cells in $F_{2}$ receive input combinations that represent subpatterns of the total activation pattern across $F_{1}$. These cells are inhibited by masking field interactions. Only the $F_{2}$ cells that respond best to the total activation across $F_{1}$ can survive these inhibitory interactions. Figures 12 and 13 illustrate this type of selectivity using computer simulations from Cohen and Grossberg (1986). These simulations illustrate that a masking field can selectively activate different $F_{2}$ cells in response to input patterns across $F_{1}$ which differ either in their spatial scale (compare Figures 12, 13a, and 13c) or in the patterning of activation across a fixed set of cells (compare Figures 13a with $13 \mathrm{~b}$, and $13 \mathrm{c}$ with $13 \mathrm{~d}$ ).

These general-purpose properties of a masking field give rise to complex cells in $F_{2}$ with desired coding properties when the simple cells of $F_{1}$ are spatially organized into alternating ocular-dominance columns containing hypercolumns whose cell-receptive fields undergo regular orientation shifts as the hypercolumn is traversed (Hubel \& Wiesel, 1977). Then the total activation pattern across $F_{1}$ unambiguously represents such factors as monocular position, binocular positional disparity, binocular orientation disparity, and spatial frequency (Figures 10 and 14). The selective response of $F_{2}$ to $F_{1}$ translates the information within the total activation pattern across $F_{1}$ into selective tuning curves for all of these factors within individual cells of $F_{2}$. In summary, although individual cells of $F_{1}$ generate positionally ambiguous responses, the spatial patterning of $F_{1}$ cell activations carries unambiguous information about position, disparity, and spatial scale. The interaction between $F_{1}$ and $F_{2}$ converts the unambiguous spatially distributed information across $F_{1}$ into unambiguous reactions of individual cells in $F_{2}$. In this way, the transformation $F_{1} \rightarrow F_{2}$ enables individual cells in $\mathrm{F}_{\mathbf{2}}$ to multiplex the data that is spatially distributed across $F_{1}$.

These masking field properties are consistent with the fact that many receptive-field properties of striate cells are interactive properties that require inhibitory interneurons for their normal expression. In particular, application of the GABA antagonist bicuculline produces striking reductions in the selectivity of striate-cell receptive-field properties (Sillito, 1974, 1975a, 1975b,
1977, 1979; Sillito, Salt, \& Kemp, 1985), notably a reduction of orientational tuning. This type of property is consistent with the model, since the divergent inputs from $F_{1}$ to $F_{2}$ are shaped by the inhibition across $F_{2}$. The model is also consistent with the fact that striate complex cells (in $\mathrm{F}_{2}$ ) exhibit true cyclopean depth reactions, whereas striate simple cells do not (Poggio et al., 1985).

Using the same mechanisms, complex cells in $F_{2}$ may multiplex an even greater number of image properties just as long as $F_{1}$ is able to represent these properties as part of its total spatial pattern of activation. Letting individual cells in $F_{2}$ receive converging inputs from $F_{1}$ cells that respond to opposite direction-of-contrast and several ranges of wavelength sensitivity (Grossberg, 1987b; Thorell et al., 1984) does not create new problems of principle, although it does raise as-yet-unanswered questions about the spatial organization of such information.

Sensitivity to spatial frequency information (De Valois et al., 1982) is a characteristic of masking field cells, whether or not the individual input cells from $F_{1}$ are sensitive to spatial frequency (Figures 12,13a, and 13c). This type of spatial frequency sensitivity enables $F_{2}$ to preferentially activate cells that best encode the total $F_{1}$ activity pattern, even if these patterns excite narrower or broader expanses of $F_{1}$ cells, as in Figure 14 . This property suggests possible differences in the mechanisms whereby simple cells and complex cells achieve spatial frequency sensitivity. Individual simple cells in $F_{1}$ may be made sensitive to lower spatial frequencies by increasing the size of their receptive fields, or input-averaging domains. Due to the organization of $F_{1}$ into ocular dominance columns, such a size increase can also cause an increase in the positional uncertainty with which networks of these simple cells respond (Figure 14). This increase in positional uncertainty can generate more widespread spatial patterns of activation across the $F_{1}$ network. The $F_{1}$ spatial pattern can, in turn, generate spatial frequency selective responses of individual $F_{2}$ cells by using the spatial frequency-sensitive $F_{2}$ properties that enable $F_{2}$ to best encode any $F_{1}$ activity pattern.

Thus, within a masking field model, the same multiplexing mechanism can be used to generate disparity-sensitive cells (Figure 10) and spatial frequency sensitive cells (Figure 14). In fact, replacing a monocular pattern (Figure 10a) with a binocular pattern (Figure 10d) is an operation formally analogous to replacing a high spatial frequency pattern (Figure 14a) with a low spatial frequency pattern (Figure 14b). This observation indicates that cells sensitive to different spatial frequencies need not, in principle, be segregated from cells sensitive to different disparities in order to achieve selective $F_{2}$ tuning curves.

In summary, the masking field model suggests how individual striate complex cells may multiplex the selective encoding of spatial position, orientation, binocular positional disparity, binocular orientational disparity, and spatial frequency, while lumping together direction-ofcontrast and wavelength sensitivity. From the perspec- 


\section{ITEM FIELD (F1)}

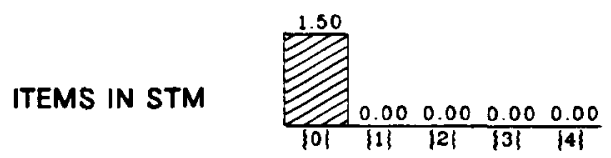

\section{MASKING FIELD (F2)}

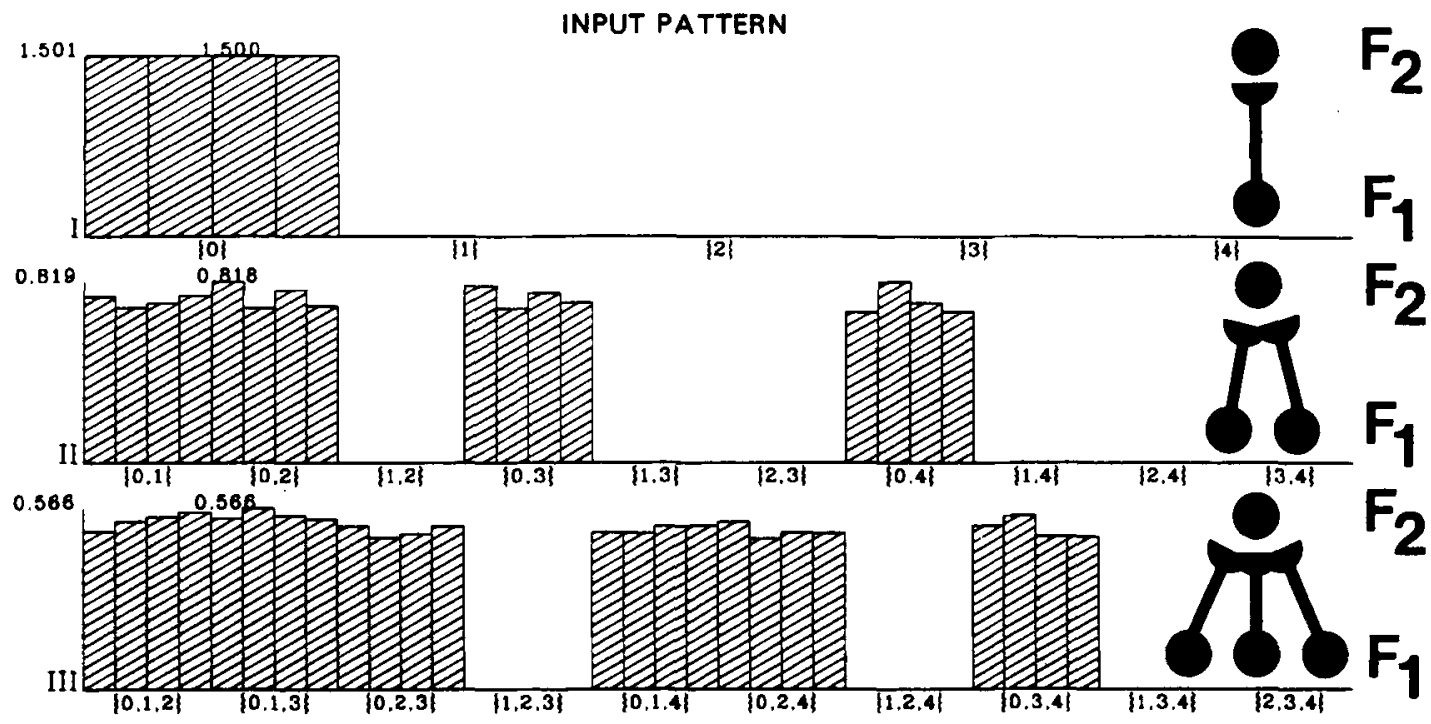

LIST CODE IN STM
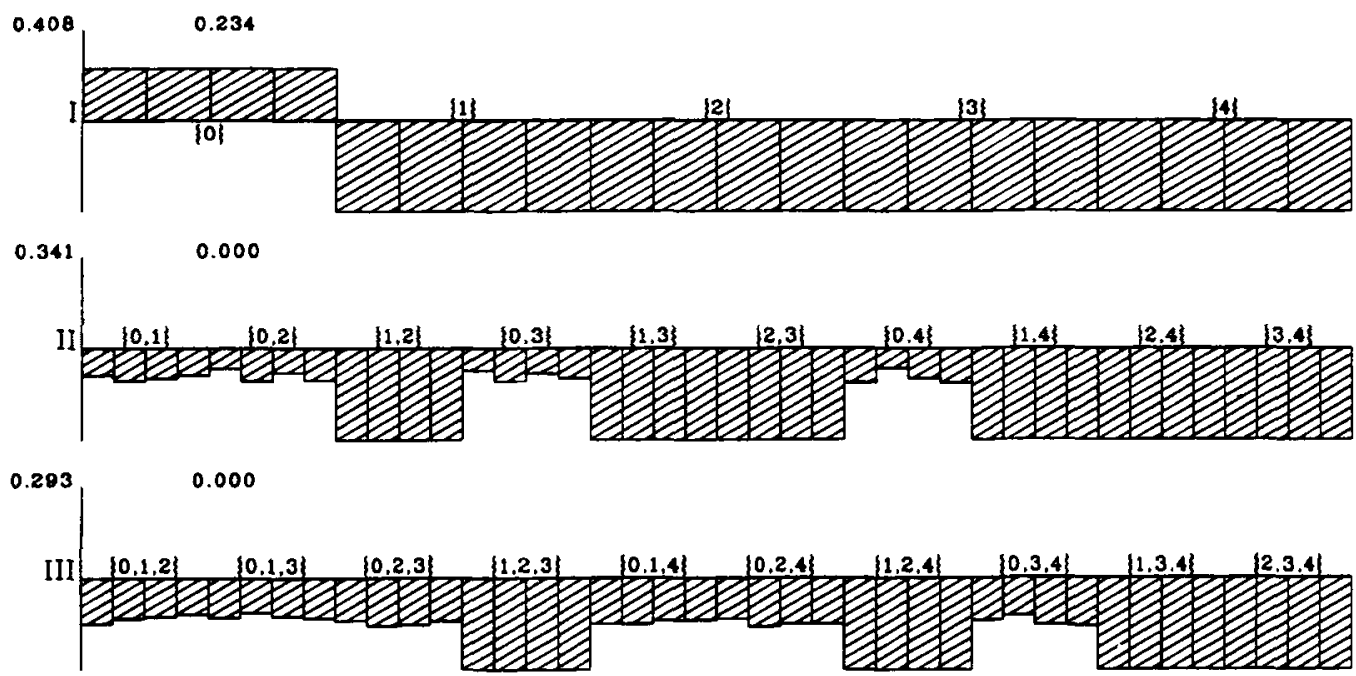

Figure 12. Multiplexing of spatial patterns of activity across $F_{1}$, into selective short-term-memory (STM) activations of individual cell populations at $F_{2}$ : The item field depicts the spatial pattern of activity across $F_{1}$. The first three rows under Masking Field, labeled Input Pattern, depict the inputs from $F_{1}$ to cells across $F_{2}$. The inputs to the cell populations of the masking field $F_{2}$ are arranged in three rows. In this computer simulation, the cell population in the ith $(i=1,2,3)$ row receive inputs from exactly $i$ populations of $F_{1}$. Thus, in row 1 , there are four cell populations in $F_{2}$, labeled $\{0\}$, which receive an input from only the population in $F_{1}$ which codes item $\{0\}$. In row 2 , there are four cell populations in $F_{2}$, labeled $\{0,3\}$, which receive an input from the populations in $F_{1}$ which code items $\{0\}$ and $\{3\}$. The number above the bars that represent input size designates the maximal input to that row. Bar heights represent input intensities. The inputs are broadly distributed across $F_{2}$ cells. The code in STM depicts the selective STM activation of the corresponding $F_{2}$ cell populations to these broadly distributed inputs. In this simulation, only a single population generates a positive response to the broadly distributed input pattern. All other activations are negative, or inhibited, and hence are not large enough to exceed the nonnegative output threshold of the cells. 
ITEM FIELD (F1)

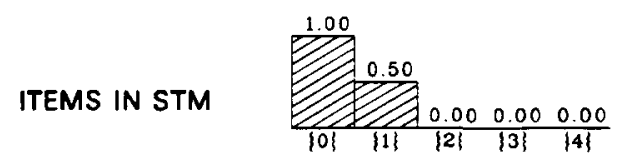

MASKING FIELD (F2)

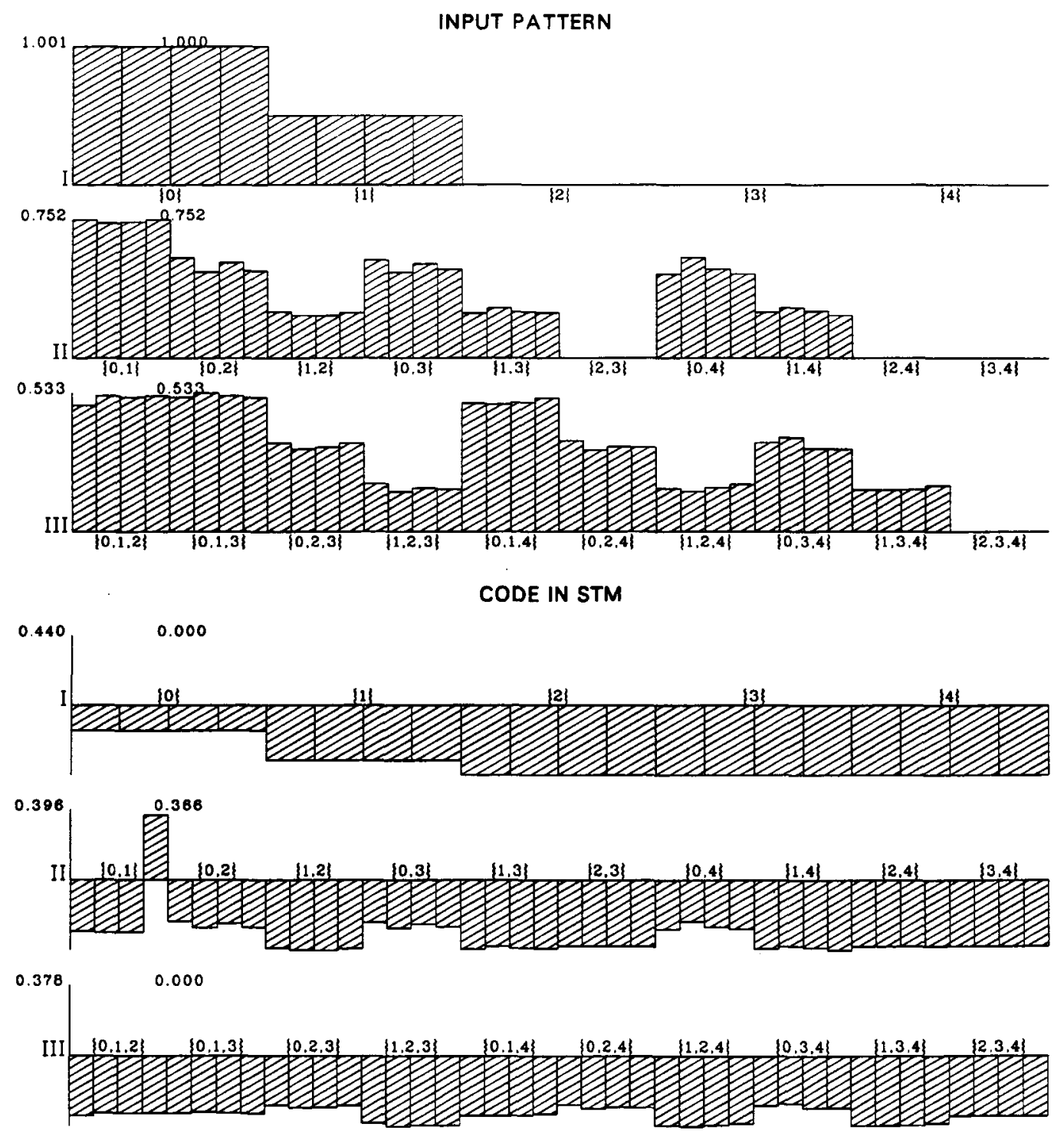

Figure 13a 


\section{ITEM FIELD (F1)}

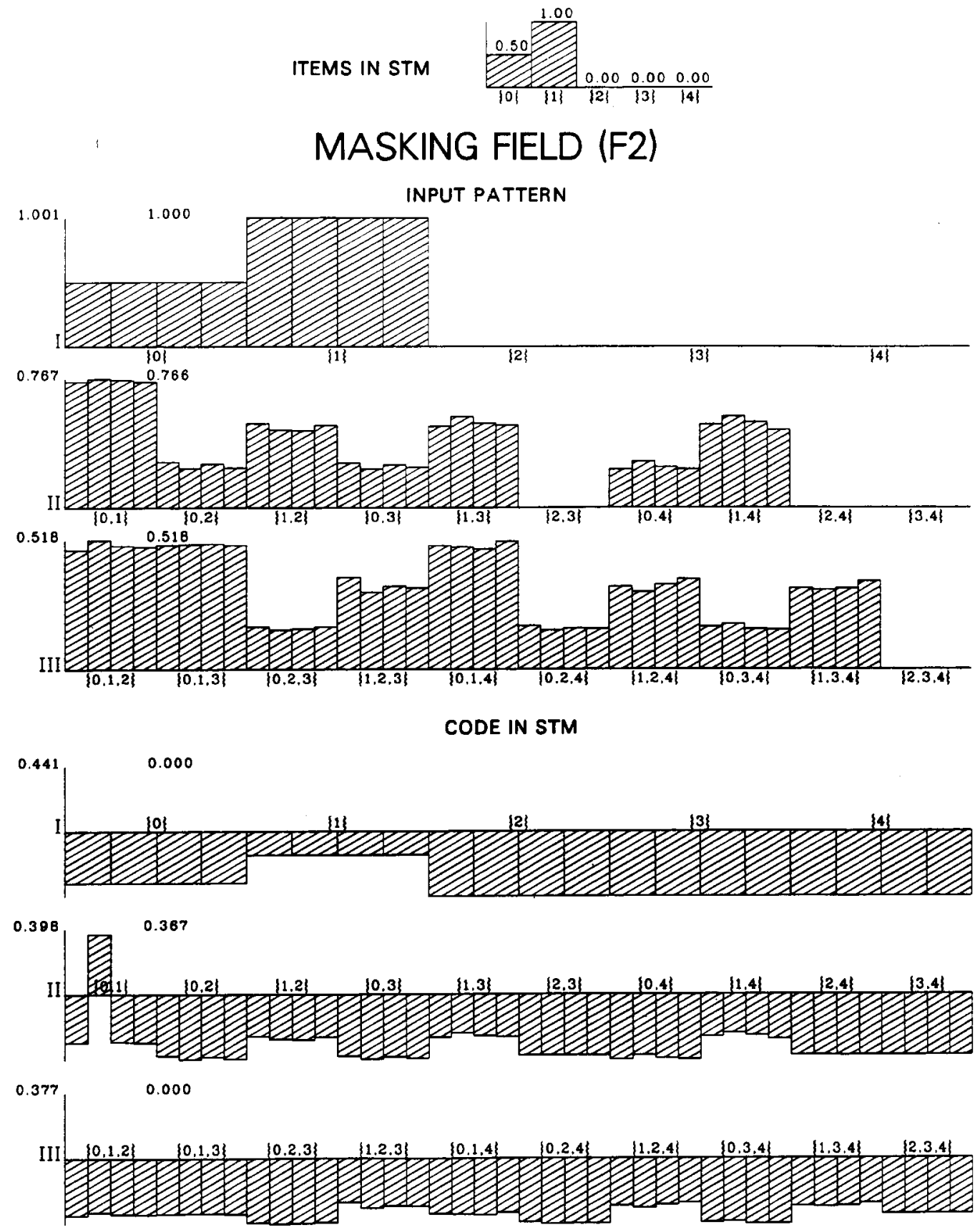

Figure 13b 


\section{ITEM FIELD (F1)}

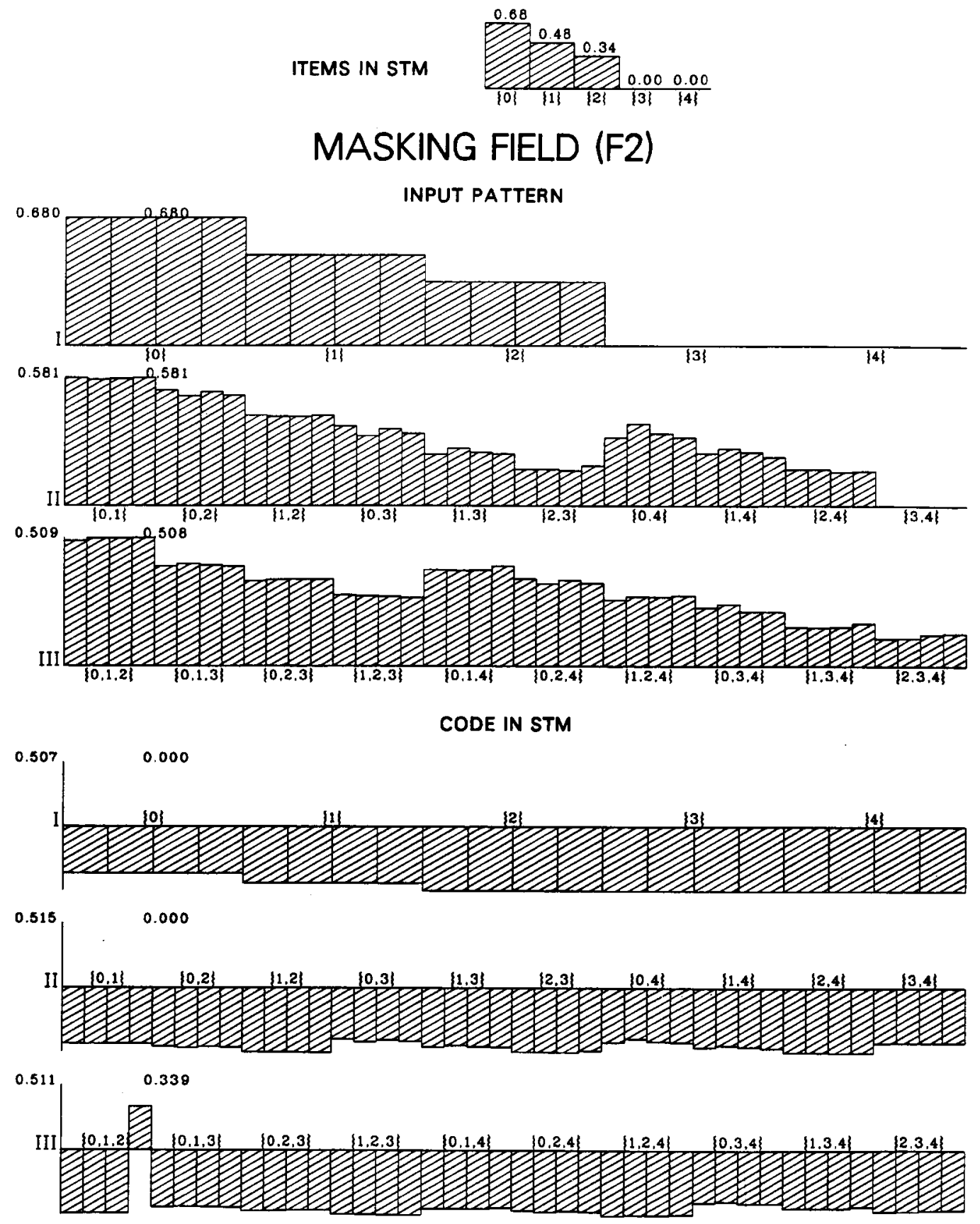

Figure 13c 


\section{ITEM FIELD (F1)}

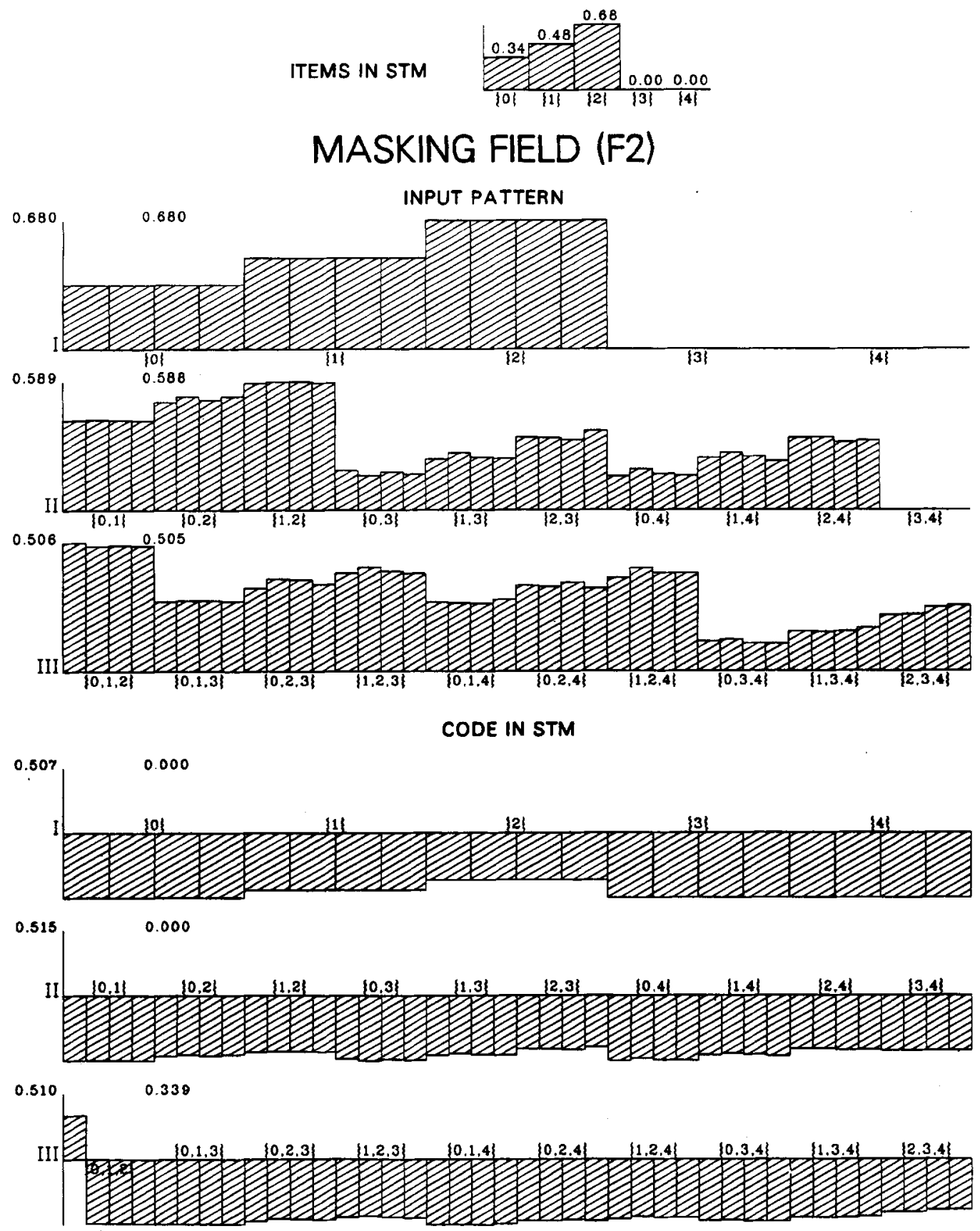

Figure 13d

Figure 13. Multiplexing of spatial patterns of activity across $F_{1}$ into selective activations of individual cell populations at $F_{2}$. In (a)-(d), four different activity patterns across $F_{1}$, varying in spatial scale and internal structuring, generate distinct selective reactions at $\mathbf{F}_{2}$. 


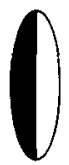

RECEPTIVE

FIELD SIZE

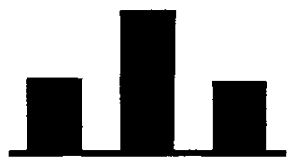

(a)

SPATIAL PATTERN

OF ACTIVATION

ACROSS OCULAR

DOMINANCE

COLUMNS

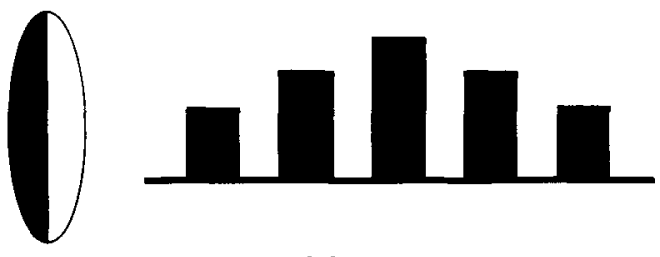

(b)

Figure 14. Early stages of spatial-frequency sensitivity are represented by covariation of receptive-field size with the spatial pattern of activity generated by cells of this size across $F_{1}$ : Small receptive fields generate more localized patterns (a) than large receptive fields (b). Bar height represents activity at each cell position. From "Neural dynamics of surface perception: Boundary webs, illuminants, and shape-from-shading" by S. Grossberg and E. Mingolla, 1987, Computer Vision, Graphics, \& Image Processing, 37, 133. Reprinted with permission.

tive of the present theory, these cells function to generate chromatically broadband, but spatially focused, spatially frequency-selective and disparity-selective binocular boundary signals to the binocular segmentation mechanisms of the CC loop.

\section{Adaptive Tuning of the Complex Cell} Filter by a Non-Hebbian Associative Law: Spatial Patterns as the Units of Learning

The third factor used by the masking field model is adaptive tuning of the $F_{1} \rightarrow F_{2}$ filter, and thus of the complex-cell tuning curves, to the activation patterns that are experienced most regularly across $F_{1}$ (Cohen \& Grossberg, 1986). Such adaptive tuning is mediated by associative long-term-memory (LTM) traces that exist at the synaptic terminals of each $F_{1} \rightarrow F_{2}$ pathway and multiply, or gate, the signals in these pathways before the gated signals can influence target cells in $\mathbf{F}_{\mathbf{2}}$. In the present application, these adaptive tuning properties form part of the machinery that regulates the critical period of developmental plasticity for binocular cortical cells (Blakemore \& Cooper, 1970; Braastad \& Heggelund, 1985; Frégnac \& Imbert, 1978; Hirsch \& Spinelli, 1970; Hubel \& Wiesel, 1977; Singer, 1985; von der Heydt et al., 1981). The theory uses an associative law that allows the LTM traces to either increase or decrease in strength in response to the correlated activities of their presynaptic $F_{1}$ neuron and postsynaptic $F_{2}$ neuron (Grossberg, 1968, 1969, 1976a).

Such an associative law does not obey the familiar Hebbian associative law (Hebb, 1949), which assumes that correlated activities always lead to an increase of associative strength. Figure 10 suggests why a Hebbian law is inadequate. Due to adaptive tuning, each $\mathrm{F}_{2}$ cell becomes more strongly and selectively activated by its "trigger" spatial pattern across $F_{1}$. All the LTM traces in pathways from $F_{1}$ to such an activated $F_{2}$ cell change as a result of the tuning process. In order for these LTM traces to learn the optimal spatial pattern of activation across the $F_{1}$ network, an LTM trace in an $F_{1} \rightarrow F_{2}$ pathway must be able to decrease if its $F_{1}$ activity is small and to increase if its $F_{1}$ activity is large. The Hebbian associative law, which mandates an increase due to associative pairing, is thus not suitable for learning spatial patterns. It was based on the idea that activities in individual pathways, rather than spatial patterns of activation across a network of cells, are the functional units of associative learning. Several investigators (Levy, 1985; Levy, Brassel, \& Moore, 1983; Levy \& Desmond, 1985; Rauschecker \& Singer, 1979; Singer, 1983, 1985) have presented neurophysiological evidence concerning cortical plasticity that are consistent with this type of nonHebbian associative law.

Suppose that such non-Hebbian LTM traces multiply the signals within the pathways converging from simple cells to complex cells, and that the complex cells interact via a masking field. Such a competitive learning system is capable of selectively tuning complex cells to the activation patterns that occur regularly across $F_{1}$ (Cohen \& Grossberg, in press; Grossberg, 1976a). For example, due to normal viewing conditions, certain complex cells may be preferentially tuned to different orientations from the left eye and the right eye (Figure 10e), if these correlations are persistent ones within their spatial scale and retinal position (von der Heydt et al., 1981). Tuning of disparity selectivity may be sharper for vertically oriented than horizontally oriented complex cells, due to the breadth of the $F_{1}$ spatial pattern that is caused by left eye and right eye representations of a horizontal contour. This property may help to explain the greater number of vertical than horizontal disparity-sensitive cells that Hubel and Wiesel (1970) found in V2, and the fact that complex cells may be either disparity sensitive (depth neurons) or disparity insensitive (flat neurons) (Poggio et al., 1985).

The existence of disparity-insensitive neurons is not considered an imperfection of cortical design within the theory. Disparity-sensitive cells are herein regarded as part of a 3-D boundary segmentation system, rather than as part of a disembodied stereopsis computation. From this perspective, it is obvious that boundaries must be computed within all spatial scales in a manner that prevents spurious featural filling-in from occurring (Grossberg, 1987b). If only finely tuned disparity-sensitive cells could input to this boundary computation, then horizontally oriented binocular cells would necessarily be excluded. The full burden of completing horizontal boundaries would then fall upon responses of the end-cutting mechanism (Grossberg, 1987b, Section 13) to nearly vertical image contrasts. Nonvertically oriented cells with lesser 
degrees of disparity-insensitivity overcome this possible problem. In particular, disparity-insensitive horizontally tuned cells can complete a rectangular boundary within a spatial scale capable of binocularly fusing the vertical boundaries of the rectangle. Such a completed rectangle could generate a filled-in representation within the corresponding syncytium within the FC System, and thereby contribute to the final percept. In contrast, disparityinsensitive horizontally tuned cells would have little influence on the syncytia of scales in which they could not complete a boundary grouping capable of supporting a filled-in percept.

This summary has suggested mechanisms capable of giving rise to the multiplexed tuning-curve properties that are characteristic of individual striate complex cells. It hereby provides enough detail to address the question of how binocular complex cells that vary in their position, orientation, spatial frequency, and disparity-sensitive properties influence a boundary completion within the BC System. Sections 2-9 indicated that the same competitive and cooperative interactions within the CC loop that were suggested in Grossberg (1987b) to complete monocularly activated boundaries are also competent to complete binocularly activated boundaries. By putting together these two lines of evidence, the theory can begin to analyze a large data base about binocular perception. I begin by refining my analysis of how these mechanisms suppress binocular double images.

Julesz (1971) and Sperling (1970) have analyzed how spurious binocular correlations between pairs of monocular images can be suppressed before they enter conscious perception. Their important pioneering models of binocular disparity detection and the suppression of spurious double images led to the active development of several models for binocular matching of edges (Dev, 1975; Marr \& Poggio, 1976, 1979). I have elsewhere discussed some basic weaknesses of these models (Grossberg, 1983b). Herein I suggest a different solution to this problem which avoids these weaknesses. The main new insight is as follows: The processes that compensate for the positional uncertainty due to orientational tuning (masking field) combine with boundary completion processes (CC loop) in such a way as to synthesize disparity-sensitive boundaries that suppress double images. Neither of these processes was incorporated into the earlier models. Hence, the present model is genuinely new.

\section{Suppression of Double Images: Self-Similar Interaction Between Mask Scale, Filter Scale, Competition Scale, and Cooperation Scale}

Four stages of network interactions lead to the suppresion of boundary signals that could otherwise have led to percepts of double images. At the first stage (simple cells), input masks average inputs over an oriented receptive field. One half of this field inhibits the other half. The output signal is rectified. Consequently, each mask can generate an output only if the net mask activity, after inhibition acts, is positive. Pairs of such input masks, each sensitive to opposite contrasts of luminance or wavelength, cooperate at the second stage (complex cells) to generate an output signal that is sensitive to position, orientation, amount of contrast, disparity, and spatial frequency, but not to direction-of-contrast.

The sensitivity to spatial frequency is particularly important for an understanding of depth perception and the suppression of false images. As mentioned in Section 22, two distinct sources of spatial frequency sensitivity are operative at the first, or input mask, stage. One source is the different sizes of the masks, or receptive fields (Figure 14), across the multiple spatial scales of the network. Despite the variability in total input mask size, the masks within all the spatial scales are designed to respond to sufficiently large and sharp contrasts in the scenic inputs that share their position and orientation. In particular, sufficiently contrastive bounding edges of a scene are monocularly detectable by oriented receptive fields in all the BC System spatial scales. Monocular detectability of bounding edges by all scales provides the most important example of how multiple scales can simultaneously be active in generating a 3-D form percept. Such multiple detectability of bounding edges "grounds" the remainder of the 3-D form computation and enables all spatial frequencies to share this feature of stereo resolution.

In contrast, only the largest masks can respond to the most gradual oriented changes in contrast. Such gradual contrast changes may occur, for example, within the interior luminance gradients of a smoothly shaded, nearby surface. Thus, the smallest masks can react to the most narrow range of spatial frequencies, in particular, to high spatial frequencies. The largest masks can react to high spatial frequencies as well as to lower spatial frequencies. This type of spatial frequency sensitivity is, in itself, insufficient to distinguish whether size differences of retinal inputs are due to different object sizes or depths (Section 11). The remaining spatial frequency sensitive mechanisms interact with the spatial frequency sensitivity of the input masks to make this distinction.

A second source of spatial frequency sensitivity at the input mask stage derives from the positional uncertainty that subserves orientational tuning of the input masks. Such positional uncertainty smoothes the input from each retinal position and can thereby, within a cortex organized into interleaved ocular dominance columns, give rise to multiple periodic receptive fields for the simple cells of the model (Figures 10 and 14). Multiple simple cell receptive fields have, for example, been reported in the experiments of Mullikin, Jones, and Palmer (1984) on cat visual cortex. The theory suggests that larger individual input masks are generated by a more widespread scatter of the input pathways corresponding to each retinal position across the field of input masks (Figure 14). This periodic distance-dependent and spatial frequency sensitive smoothing of the input provides a framework for rationalizing the successful use of the Gabor transform for analysis of the spatial frequency properties of visual cortex (Daugman, 1980; Gabor, 1946; Kulikowski \& Kranda, 
1986; Kulikowski, Marčelja, \& Bishop, 1982; Pollen, Andrews, \& Feldon, 1978; Pollen \& Ronner, 1975, 1981, 1983).

I assume that the adaptive filter that carries outputs from the first stage, $F_{1}$, to the second stage, $F_{2}$, is self-similar: that is, larger receptive fields at $F_{1}$ can broadcast their outputs across a broader expanse of cells at $F_{2}$. As a result of this correlation, there exist disparities at which pairs of large input masks can converge upon individual binocular cells at $F_{2}$, although pairs of small input masks cannot converge upon individual binocular cells at the second stage (Figure 15). In other words, there exist disparities at which large input masks activate a single spatial locus of cells in the masking field, whereas small input masks activate a pair of disparate spatial loci in the masking field. By combining the different spatialfrequency sensitivities of input masks and binocular cells, the following conclusion may be drawn: Image contrasts that are sharp enough to activate a pair (left eye, right eye) of high-frequency input masks may be too disparate to activate individual binocular complex cells. At the same disparity, image contrasts that are either sharp or gradual may activate a pair of low-frequency input masks that can activate individual binocular complex cells. This interaction begins to correlate size differences with disparity differences, but it does not yet cope with the many pairs of left and right monocular input masks that cannot acti-

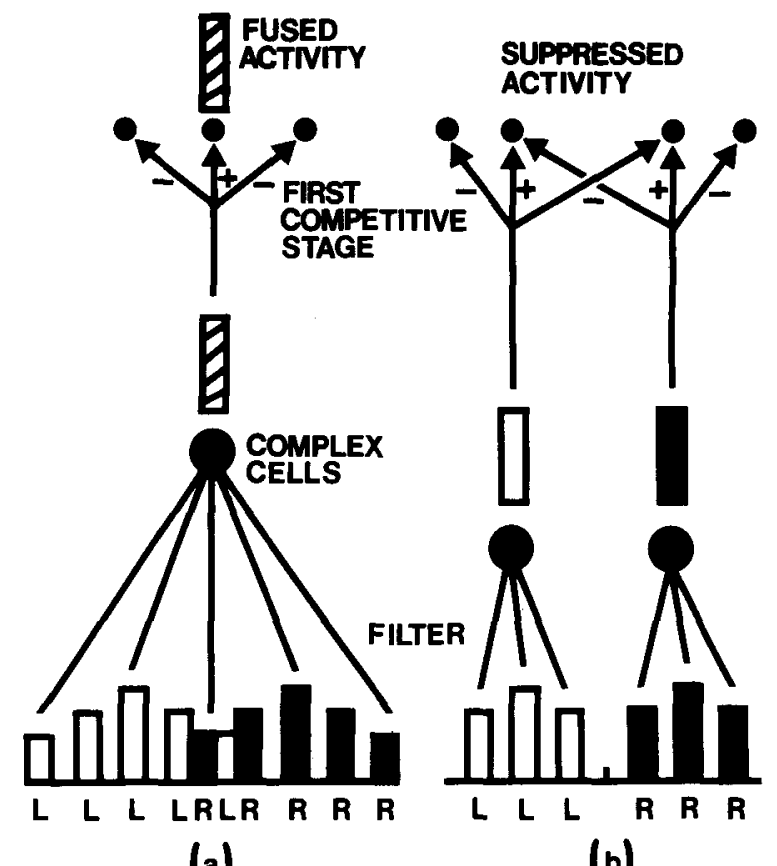

(a) (b)

Figure 15. Early stages of binocular fusion and rivalry: A low spatial frequency channel in (a) can binocularly fuse a pair of monocular images at a fixed disparity that cannot be fused by the high spatial frequency channel in (b). The fused input in (a) generates a focal excitation at the first competitive stage. The disparate monocular inputs in (b) can cancel each other at the first competitive stage. These circuits show how the distinction made in Figure 6 can be mechanistically realized. vate individual binocular cells. The CC loop solves this problem as follows.

Outputs from the masking field input to the first competitive stage. A single spatial locus of activation at the masking field can excite a single spatial locus within the corresponding spatial scale of the first competitive stage of the CC loop (Figure 15a). In contrast, a pair of disparate spatial loci at the masking field can mutually inhibit each other's signals using the first competitive stage (Figure 15b). Thus, the first competitive stage can suppress the boundary signals due to pairs of monocular boundary signals that are incapable of fusing at the masking field stage (double images). If, for any reason, one of a pair of disparate masking field activations is stronger than the other, then its BC signal can inhibit the other one at the first competitive stage.

In summary, a self-similar hierarchical interaction between multiple-scale input masks, masking fields, and short-range oriented competition sets the stage for computing fused binocular boundaries versus rivalrous monocular boundaries, thereby mechanistically explicating the concept of binocular filter depicted in Figure 6. Moreover, although all spatial scales can respond well to high spatial frequency contrasts, the larger scales can fuse large binocular disparities that the smaller scales cannot fuse. All of the interactions summarized above use relatively short-range spatial interactions. As indicated in Section 4, feedback interactions between the short-range competition and the long-range cooperation of the CC loop endows the selection of binocularly consistent boundaries and the suppression of binocularly discordant boundaries with properties of completion and coherence. In Section 20 of Grossberg (1987b), it was further suggested that the bipole cells of the CC loop exhibit a property of self-similarity that enables them to fire only in response to enough scenic evidence. In this section, it was suggested that the simple cells and their $F_{1} \rightarrow F_{2}$ pathways within the $\mathrm{OC}$ filter also possess a self-similarity property that helps to distinguish between object size and object depth. The property of self-similarity may thus be a rather general principle of cortical design that is worthy of more experimental investigation.

Many types of data relating stereopsis mechanisms to emergent boundary segmentation mechanisms are clarified by the binocular BC System design that is summarized above. For example, Russell (1979) reanalyzed the data of $\mathrm{Lu}$ and Fender (1972), who studied the chromatic input to stereopsis by using pairs of random dot stereograms built up from arrays of small colored squares. These authors varied the luminance of all squares of one color until depth was perceived. Russell (1979) concluded that "depth was perceived when there was enough signal from the enhanced edge detector [read: chromatically broadband boundary detector] to allow the stereopsis process to calculate depth [read: generate emergent binocular boundaries] from the disparity of the edges detected"' (p. 834). Russell calculated that the opponent L-M signal is an important component of this boundary detec- 
tor, which is consistent with the discussion of complex cell properties in Section 31 of Grossberg (1987b).

Earle (1985) has shown that the "introduction of stereoscopic depth effects can destroy the perceptual salience of both local and global Glass pattern structure present in a two-dimensional projection..., can be used to create novel three-dimensional Glass patterns...., and can lead to the perception of both local and global Glass pattern structune when none is apparent in the twodimensional projection" (p. 551). Such data can be explained as the result of several interacting BC System processes, notably the $\mathrm{OC}$ filter and short-range competitive processes through which binocular viewing can selectively fuse binocular boundaries from spatially disparate pairs of monocular boundaries, parse the fused boundaries into the CC loop spatial scales capable of supporting the fusion, and initiate scale-specific boundary completion and grouping with the other binocular boundaries that are processed within that scale. Earle went on to say that his data "are in contradiction of the [primal sketch] proposals advanced by Marr”' (p. 551).

\section{Interaction of Binocular Boundaries with Monocular Syncytia During \\ Binocular Fusion and Rivalry}

Having provided a framework for discussing how binocular boundary signals are selected and how they generate outputs from the BC System to the FC System, I can now deepen the analysis of how these boundary signals selectively enable some, but not necessarily all, FC signals to generate the filling-in events that lead to visible percepts. This analysis suggests several new functional reasons why the FC System is organized into networks of double-opponent cells interleaved with networks of syncytia. It also supports a neural model of double-opponent color fields that takes the form of a specialized gated dipole field (Grossberg, 1976b, 1980). I also show that, although individual double-opponent cells may have unoriented receptive fields, when a network of such cells processes oriented BC signals and oriented scenic contrasts, it acts like a form-selecting mechanism, not merely as a source of veridical color signals. Thus, the several stages of the FC System elaborate progressively more sophisticated properties of color and form together due to their interactions with the BC System. These properties are thus emergent properties of FC System and BC System interactions, and cannot be understood by an analysis of either system as an independent module.

The starting point of this analysis was outlined in Sections 17 and 18. In Section 17, I suggested how a binocular BC signal can generate both filling-in generators (FIGs) and filling-in barriers (FIBs). It was shown how monocular FC signals to the monocular syncytia can be prevented from eliciting output signals unless they interacted with topographically matched FIGs. Thus although FC signals are topographically broadcast to the syncytia that process their wavelengths across all spatial scales, only scales in which a topographic match with boundary signals from the BC System occurs can elicit output signals to the binocular syncytium.

In Section 18, I noted that even the existence of output signals from a spatial scale of a monocular syncytium does not guarantee that a percept will be generated by that spatial scale. Within each scale of a binocular syncytium, pairs of FC output signals from the corresponding monocular syncytia of the left and right eyes are topographically matched. Approximately matched FC signals can binocularly summate. A monocular FC signal in the absence of any input from the other eye can also be registered by the binocular syncytium. However, pairs of FC signals that are spatially too disparate, or mismatched, can inhibit each other before they can activate the binocular syncytium. This type of lateral inhibition is mediated by the organization of the binocular syncytium into double-opponent cells: Spatially mismatched FC signals of like wavelength can inhibit each other due to the on-center off-surround interaction among like wavelengthsensitive cells within a double-opponent field.

In summary, Sections 17 and 18 described two different ways in which disparity-sensitive processes can contribute to a percept of form-and-color-in-depth. I now show that these interactions of binocular BC signals with multiple-scale monocular and binocular syncytia also possess other properties that help to explain difficult perceptual data if the syncytia are interleaved with networks of double-opponent cells.

A core issue can be stated as follows. Suppression of double images is a fundamental property of binocular vision. Thus, it is an appealing idea that output signals from the BC System to the FC System occur only after the BC System has already undergone a binocular matching process that internally suppresses many boundaries that could otherwise have supported percepts of binocular double images (Section 24). A formidable difficulty could arise from the very property of the BC System that seems so desirable. The process of suppressing binocular double images synthesizes a single coherent binocular boundary segmentation. If, however, a single binocular boundary structure outputs to the FC System, how can this segmentation simultaneously suppress a percept from one eye while supporting a percept from the other eye, as occurs during binocular rivalry? Moreover, even if one wished to overturn the idea that a single binocular BC System exists, how could one then explain the large data base concerning the preponderance of binocularly tuned orientation-selective cells at both early and late stages of visual cortical processing?

This issue has already drawn the attention of several visual scientists (Cogan, 1982; Savoy, 1984). Savoy has provided a particularly clear discussion of the issue as a basis for some of his important experiments on interocular transfer of the McCollough effect. These experiments led Savoy to reject a model in which a single "binocular achromatic spatial system"' (read BC System) inputs to a pair of monocular "pre-color systems" (read FC System). Savoy (1984) went on to write: "There is also no 
way to account for binocular rivalry in either spatial or color systems .... Some of these connections might have other implications, such as might be relevant to another major issue that is beyond the scope of the present class of models-stereopsis"' (p. 575).

I support Savoy's intuitions below by showing how a theory capable of analyzing binocular rivalry and stereopsis can also account for interocular transfer properties of the McCollough effect (Section 29). On the other hand, I do this using a variant of the very type of model that Savoy has rejected. The possibility of reconciling these ostensibly contradictory elements arises from the detailed properties of the microprocesses and hierarchical interactions of the BC System and FC System, notably from the way in which binocular FIGs and FIBs regulate fillingin syncytia interleaved with double-opponent fields.

To see how to proceed, let us focus upon the following example. Consider a time when the left-eye input due to a Kaufman stereogram (Section 2) is being suppressed due to binocular rivalry. The $45^{\circ}$-oblique dark parallel lines of the left image are then suppressed while the $135^{\circ}$ oblique dark parallel lines of the right image are visible. In Section 4, I argued that, when this happens, the $135^{\circ}$ oblique BC signals suppress the $45^{\circ}$-oblique BC signals within the CC loop. Then the $135^{\circ}$-oblique BC signals generate FIGs to both the left monocular syncytium and the right monocular syncytium (Section 17). Why cannot the $135^{\circ}$-oblique FIGs generate output signals from the left monocular syncytium in response to its $45^{\circ}$ oblique FC input signals? Why can the $135^{\circ}$-oblique FIGs generate output signals from the right monocular syncytium in response to its $135^{\circ}$-oblique FC input signals? Since the syncytial interactions within the monocular syncytium are unoriented, how can the FC System act as if it contained orientation-specific cells when it receives oriented BC signals? Figure 16 schematizes my solution of this basic problem.

Opponent processing occurs among the wavelengthsensitive FC cells that input to the monocular syncytium. Consequently, a spatial contrast in the wavelengths to which the cells are sensitive can cause an FC ON input as well as a spatially contiguous FC OFF input (Figure 16a). These ON and OFF signals generate inputs to distinct monocular ON and OFF syncytia, where they initiate featural filling-in. If a FIG inputs to a syncytial position that is located between the ON and OFF FC inputs, then filling-in due to the ON signal will occur to one side of the FIG and filling-in due to the OFF signal will occur to the other side of the FIG. If no FIG signal is interpolated between the $\mathrm{ON}$ and $\mathrm{OFF}$ inputs, however, then filling-in due to both signals will spread over similar positions within the ON and OFF syncytia.

Each syncytium gives rise to topographically organized output signals. The output signals of ON and OFF syncytia compete at each position before generating a net FC output signal to the binocular syncytium (Figure 16b). If a FIG is spatially interpolated between the ON and OFF input signals, then a net FC ON signal can be generated

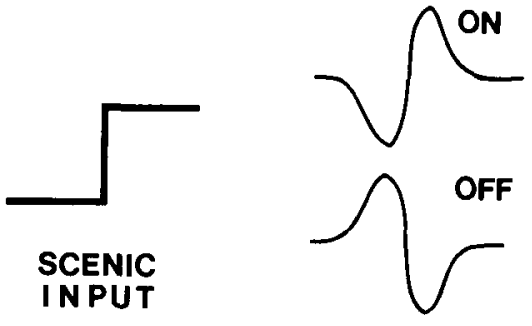

(a)

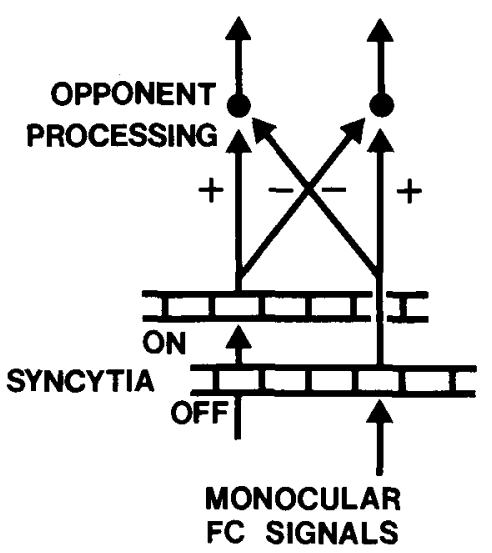

(b)

Figure 16. Spatial correlation between ON and OFF FC input signals with FIG signals determines whether FC output signals will be generated: (a) A spatial discontinuity in the scenic input pattern causes correlated $\mathrm{ON}$ and $\mathrm{OFF}$ reactions within opponent FC input pathways. (b) These opponent reactions excite opponent ON and OFF syncytia at spatially contiguous positions. Lateral filling-in due to these $O N$ and $O F F$ inputs occurs within the $O N$ and $O F F$ syncytia (connected rectangles) except across locations where FIG inputs are registered. Opponent competition between the ON and OFF syncytia at each position determines the net FC output signal.

to the binocular syncytium (Figure 17a). If no FIG interpolates the ON and OFF input signals, then the ON FC output signal can be inhibited by opponent competition from the filled-in activity pattern within the OFF monocular syncytium (Figure 17b).

Figure 17 shows how opponent processing at each position of the inputs and outputs of the ON and OFF syncytia can generate output signals or not, depending upon the location of a FIG input signal to both syncytia. Figure 18 uses these properties to help explain the rivalrous percept seen during inspection of the Kaufman stereogram, and more generally the nonselective nature of rivalry suppression. Two cases arise: The boundary synthesized by the BC System is either perpendicular to or parallel to the contour of ON and OFF FC inputs received by the monocular syncytia.

When the FIG signals and the FC input signals are spatially in-phase (Figure 18a), the ON monocular syncytium can generate FC output signals to the binocular syncytium all along the extent of the FIG, as in Figure 17a. When the FIG signals and the FC input signals are perpendicu- 
lar (Figure 18b), the filled-in activities within the $O N$ and OFF syncytia can mutually inhibit each other's output signals via opponent processing, as in Figure $17 \mathrm{~b}$. Thus, although the receptive fields of individual syncytial and opponent processing cells may be unoriented, networks of these cells respond to oriented combinations of FC and $\mathrm{BC}$ input patterns with orientation- and form-sensitive properties. In particular, if the boundary selected at a given moment by the BC System parallels the monocular FC data from a given eye, then these data can generate input signals to the binocular syncytium for further processing into a visible percept. If this boundary is perpendicular to, or even sufficiently oblique, with respect to the monocular FC data from the other eye, these data are suppressed before they can input to the binocular syncytium. If the orientation of the boundary flips due to habituative-competitive-cooperative interactions within the CC loop (Section 9), then the previously suppressed eye's FC data can activate the binocular syncytium.

\section{Interaction of Double-Opponent Networks with Binocular Boundary Signals}

The opponent processing properties illustrated in Figures 16-18 can be used to analyze a variety of paradoxical psychophysical data about binocular rivalry and the McCollough effect. To form a bridge to these data, it is first necessary to integrate the opponent processing
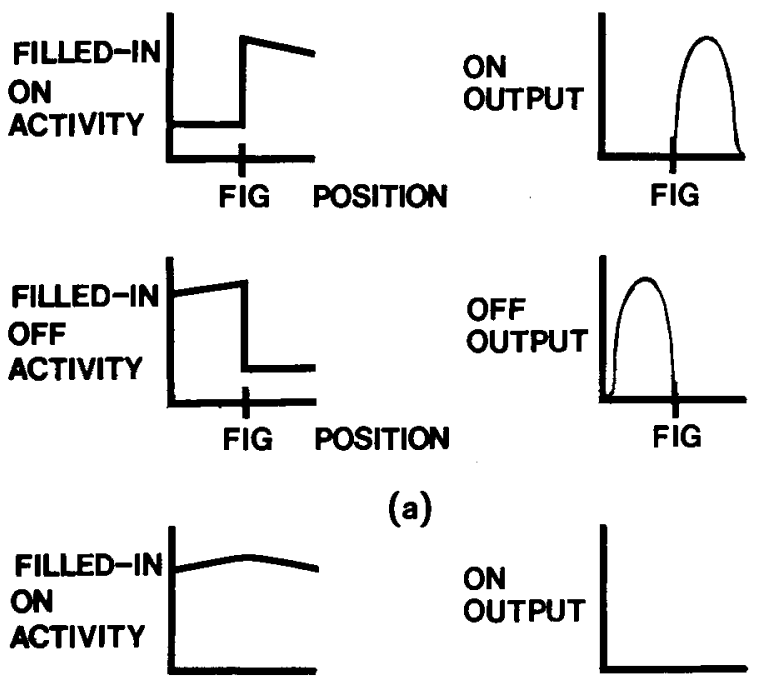

(a)
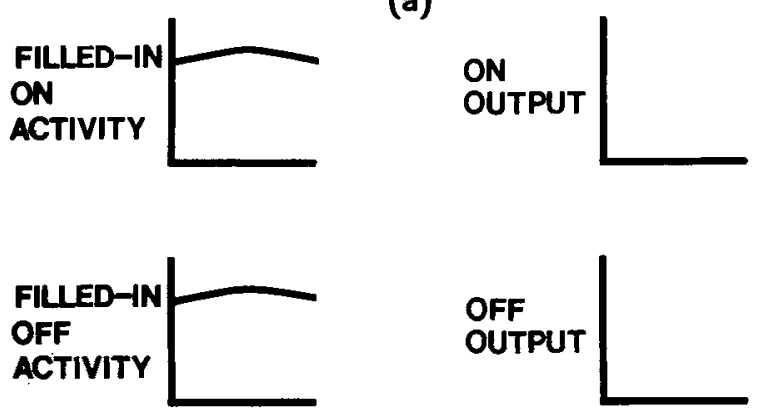

(b)

Figure 17. Opponent processing of filled-in activity patterns in on- and off-syncytia generates spatially adjacent on- and off-output signals if a FIG signal is properly placed, as in (a), but not if fillingin proceeds unimpeded, as in (b).

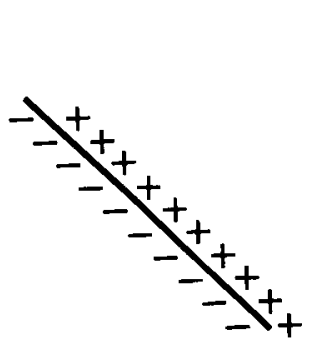

(a)

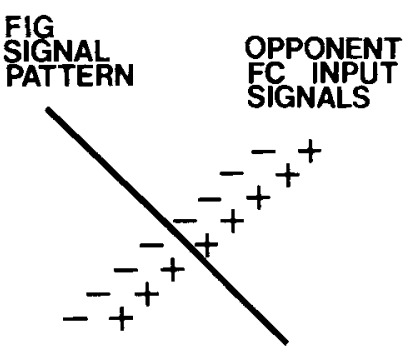

(b)
Figure 18. Two-dimensional overlap of a FIG contour and a contour of correlated ON and OFF inputs to opponent ON and OFF syncytia determines whether output signals will be emitted by the syncytia: (a) If a FIG input (solid line) interpolates ON $(+)$ and OFF (-) inputs, FC output signals from the ON syncytium and the OFF syncytium, respectively, can be generated from opposite sides of the FIG. (b) If $O N(+)$ and $O f f(-) F C$ inputs are not interpolated by a FIG input (solid line), the filled-in activities within the $\mathrm{ON}$ and OFF syncytia can cancel each other's output signals.

properties of the ON and OFF syncytia into a more complete scheme of double-opponent processing. This can be done by combining the analyses of monocular syncytial interactions found in Sections 17 and 25.

Section 17 argued that spatial discontinuities of the filled-in activity pattern across a monocular syncytium can generate FC output signals by being passed through a shunting on-center off-surround network filter. This type of lateral inhibition prevents the filter from generating outputs to the binocular syncytium unless a FIG is present to generate such a spatial discontinuity. Shunting lateral inhibition across positions and within a single syncytial channel mediates this property. Section 25 argued that, in addition, subtractive lateral inhibition within positions and across opponent syncytial channels mediates spatially correlated $\mathrm{ON}$ and $\mathrm{OFF}$ reactions. These reactions prevent the filter from generating outputs to the binocular syncytium unless a FIG interpolates the spatial pattern of ON and OFF FC inputs to the monocular syncytium. This total network architecture for processing the inputs and outputs of the monocular syncytium obeys the rules of a gated dipole field: shunting on-center off-surround interactions occur within the ON subfield and within the OFF subfield; subtractive opponent interactions occur between positionally matched ON and OFF cells.

If a pair of ON and OFF subfields is identified with an opponent channel for red-green, blue-yellow, or whiteblack processing, then such a gated dipole field possesses the properties of a network of double-opponent cells. For example, identifying red with the ON channel and green with the OFF channel enables us to conclude that a red ON activation inhibits nearby red $\mathrm{ON}$ activations via shunting lateral inhibition within the $O N$ subfield. In addition, a red ON activation also inhibits a green OFF activation at the same position via subtractive opponent inhibition. Thus, our analysis of the functional properties needed to suppress binocularly discordant FC signals has 
led to a totally new viewpoint for understanding why color processing is organized into networks of double-opponent cells. This analysis also suggests that three pairs (redgreen, blue-yellow, black-white) of monocular syncytia and double-opponent networks exist within each spatial scale of the FC System, and that FIG signals from each spatial scale of the BC System are topographically registered at all six monocular syncytia of that spatial scale.

The interpretation of ON and OFF processing in terms of double-opponent cells suggests that the terms $O N$ and OFF are relative. For example, a red (green) signal is an ON signal to its own syncytium and an OFF signal to the green (red) syncytium. Only in the case of the chromatically broadband white-black system does the terminology $\mathrm{ON}$ and OFF have an absolute significance, since only the white system is activated by external inputs. Output signals from the black system are activated due to disinhibition of internally generated tonic actvity. Thus, although there exists a tendency to symmetry in the double-opponent network of red versus green and blue versus yellow, the white versus black system contains a manifest asymmetry due to the fact that only the white subsystem is activated by external inputs. One manifestation of this asymmetry was observed by Livingstone and Hubel (1984a), who reported that "on-center cells outnumbered off-center cells by more than two to one" (p. 321).

Modeling double-opponent networks as gated dipole fields also clarifies how negative aftereffects of color or luminance can be generated by antagonistic rebounds due to sudden offset of previously sustained inputs (Grossberg, 1976b, 1980). Correlated rebounds within the gated dipole fields of the CC loop (Section 9) and the gated dipole fields of the FC System are both needed to initiate the boundary completion, double-opponent, and filling-in events that organize the negative afterimage into a visible percept.

In summary, each $\mathrm{FC}$ input to the monocular syncytium is topographically broadcast to all the subsyncytia, across all spatial scales, which process its wavelength. Thus, red FC inputs activate the red syncytia of all spatial scales. In contrast, each FIG is topographically broadcast to all the syncytia within its spatial scale, irrespective of their wavelength sensitivity. Thus, a FIG output from a high spatial frequency BC System scale is registered at all the (red, green), (blue, yellow), and (white, black) syncytia corresponding to that spatial scale. Then double-opponent networks process the outputs from all pairs of opponent syncytia on their way to the binocular syncytium.

\section{Psychophysical Properties of Binocular Rivalry}

It has been shown that the depth of suppression during binocular rivalry is independent of a variety of properties of the contralateral eye's rivalry target, such as its orientation (Blake \& Lema, 1978), its contrast (Blake \& Camisa, 1979), its luminance (Hollins \& Bailey, 1981), and the time during the suppression phase when a test probe is presented (Fox \& Check, 1972). These data refine the conclusion of Wales and Fox (1970) that "rivalry suppression ... nonselectively attenuates all classes of inputs falling within the spatial boundaries of the suppressed target"' ( $p 90$ ). On the other hand, the average duration of a suppression phase varies inversely with the strength of the suppressed image. Doubling the contrast of a rivalry stimulus can almost halve the length of time that the image is suppressed (Blake, 1977), and adding strength to an already suppressed image can abbreviate the duration of suppression (Blake \& Fox, 1974). A viable theory of binocular rivalry must explain how a variable duration of suppression can coexist with a constant depth of suppression over a wide range of stimulus conditions.

The present theory explains the variable duration of suppression as a consequence of five interacting factors. First, the oriented receptive fields of the BC System are sensitive to amount-of-contrast near scenic edges. Thus, more contrastive scenic edges generate larger BC System signals, other things being equal. Second, the rate of habituation of the transmitter gates in active feedback pathways of the CC loop (Section 9) depends upon the size of the signals in these active pathways. Habituation takes place more rapidly if the signals are larger. Thus, CC loop pathways that are activated by larger contrasts will tend to habituate faster. This property clarifies why switching rate is sensitive to contrast, but it also brings into focus the mechanistic issue that makes these data hard to explain.

Why does not the amount of rivalry suppression also vary continuously with the amount of habituation? How can habituation take place continuously and in an activitydependent fashion, yet switching itself be an all-or-none process? These questions motivate my third point. The property of all-or-none switching is controlled by the nonlinear feedback dynamics of short-term-memory storage within the CC loop. The transmitter gates multiply the positive feedback signals to the competing orientations within the CC loop (Section 9). As a transmitter gate habituates within its active pathway, the amount of positive feedback that the pathway supplies to its orientation decreases continuously through time. On the other hand, this process is only one of several factors that control the switching properties of the CC loop. The feedback interactions that enable the CC loop to overcome initial orientational uncertainty and to choose a globally sharp and coherent boundary also endow the CC loop with properties of hysteresis. Consequently, the winning orientations do not gradually shut off due to habituation; rather, a time is reached when the cumulative habituation of the transmitter gates in the active pathways has so attenuated the transmitter-gated feedback signals in these pathways that the network's hysteresis is suddenly overcome. The previously suppressed orientations can then win the competition and begin to cooperatively group into a new boundary configuration. Extensive computer and mathematical 
analyses of all-or-none switching due to continuous habituation of transmitter gates can be found in Carpenter and Grossberg $(1983,1984,1985)$, where a physically distinct, but functionally related, model of a gated dipole field is characterized. In summary, although the transmitter habituation within the CC loop is continuous, its switching behavior is discrete.

Fourth, the FIG signals are nonlinear functions, in fact S-shaped functions, of BC System activity (Cohen \& Grossberg, 1984a). After a boundary segmentation becomes active within the CC loop, its FIG signals to the FC System approximate binary on-off signals. Fifth, whenever FIG signals do not interpolate the spatial pattern of FC inputs to the monocular syncytium, the output signals from the syncytium are nonspecifically attenuated (Section 25). In particular, Figure 18 illustrates that all orientations of monocular FC-input patterns that are not parallel to, and even interpolated by, a FIG contour are suppressed. Thus, due to interactions between the BC System and the FC System, binocular rivalry can exhibit perceptual properties of nonspecific suppression even though the computations within the BC System are oriented.

Unoriented suppression may also arise directly from BC System properties. The first competitive stage inhibits like orientations at nearby positions, whereas the second competitive stage inhibits perpendicular, and close-toperpendicular, orientations at the same position (Figure 2). Thus, a rivalry paradigm that does not control the relative spatial phases of its images may generate inhibition that seems to be unoriented because the combined effects of both competitive stages in response to such a paradigm can inhibit a broad band of orientations.

In summary, the theory suggests explanations for a number of important psychophysical results concerning binocular rivalry, using properties whereby the BC System generates sharp coherent boundaries with hysteretic properties that selectively attenuate binocularly discordant features via interactions with FC System filling-in syncytia interleaved with double-opponent networks. Other properties of rivalry are explained below, using the same mechanisms.

\section{Chromatic Suppression and Achromatic Sparing During Binocular Rivalry}

Smith, Levi, Harwerth, and White (1982) have described psychophysical data concerning the suppression of colored flashes during binocular rivalry. They discovered that binocular rivalry nonspecifically attenuates opponent-color information but spares achromatic information. To clarify the issues raised by their data, a brief summary of the experiment is given.

Their rivalry stimuli were high-contrast square-wave gratings that had a fundamental spatial frequency of 2.8 cycles per degree and were presented separately to the two eyes at perpendicular orientations of $45^{\circ}$ and $135^{\circ}$. A rectangular test stimulus, $.4^{\circ} \times .8^{\circ}$ in size, was projected as $20-\mathrm{msec}$ flashes to the center of the left eye. An ascending method of limits was used to estimate de- tection thresholds for each of three viewing conditions: (1) monocular nonrivalry-the right eye was occluded with a black patch; (2) binocular rivalry during the lefteye dominance phase; and (3) binocular rivalry during the left-eye suppression phase. The spectral sensitivity functions for the nonrivalry control condition and the dominance phase of binocular rivalry were similar, with three sensitivity peaks at about $440 \mathrm{~nm}$ (blue), $530 \mathrm{~nm}$ (green), and $610 \mathrm{~nm}$ (red). The spectral sensitivity curve during the suppression phase of binocular rivalry was unimodal with a single broad peak near $550 \mathrm{~nm}$ and a shape that fit the mean spectral sensitivity function determined by a flicker method under nonrivalry conditions. This sensitivity function implicates an active achromatic channel during the suppression phase.

The nonspecific attenuation of double-opponent signals can be explained as in Section 26. The sparing of achromatic signals raises a number of subtle issues concerning differences between the temporal and spatial parameters of chromatic and achromatic channels and their interactions even at early processing stages (King-Smith, 1975). I have already mentioned herein one theoretical property that may significantly contribute to this result: the asymmetry within the white-black achromatic system that was noted in Section 26. If OFF signals from the black syncytium cannot completely inhibit ON signals from the white syncytium, then FC signals may be generated from an achromatic monocular syncytium, even if no FIG is collinear with the achromatic input. On the other hand, in order for a FC output signal to be generated from the white syncytium, the filled-in activity within the white syncytium due to the test flash must be significantly larger than the resting syncytial activity within the white syncytium on the other side of some FIG. This is true because of the basic property that a syncytium cannot generate an output signal unless there is a spatial discontinuity in its filled-in activity pattern. This FIG may be generated by activation of the other eye and need not be collinear with the test flash. The explanation thus uses, in an essential way, the property that binocular FIG signals reach the monocular syncytia of both eyes.

The explanation also clarifies why varying properties such as the duration of a brief test flash during the suppression phase does not significantly influence its detectability (Hollins \& Bailey, 1981; Wales \& Fox, 1970). The criterion for detectability is essentially "structural": Is an FC output signal generated from a monocular syncytium after syncytial filling-in and double-opponent competition occur? Unless the flash can overcome the hysteresis of the active binocular boundary, the answer will not substantially change.

\section{The McCollough Effect: Monocular and Binocular Properties}

In this section, I use the same mechanisms to suggest a unified explanation of the following phenomena: why, under monocular presentation conditions, the McCollough effect does not transfer interocularly (McCollough, 1965; 
Murch, 1972; White, Petry, Riggs, \& Miller, 1978); why subsequent viewing of achromatic gratings of the same spatial frequency and orientation speeds up the decay, or “extinction,"' of the effect (Savoy, 1984; Skowbo, Timney, Gentry, \& Morant, 1975); why this type of "extinction" does not transfer interocularly (Savoy, 1984); and why the McCollough effect depends upon the duration of exposure rather than upon the number of exposures to inducing stimuli (Skowbo \& White, 1983). All of the data emphasize monocular properties of the McCollough effect. Explaining the McCollough effect is complicated by the following types of binocular properties.

Vidyasagar (1976) has shown that individuals may be trained to experience opposite McCollough effects in response to monocularly and binocularly presented images. White et al. (1978) have reported four experiments that discovered a number of demanding binocular effects. In Experiment I (Figure 19A), they compared binocular rivalry and no-rivalry conditions of inspection. In both conditions, subjects focused upon a fixation point as their left eyes were exposed to magenta verticals alternating with green horizontals. In the no-rivalry group, the right eye was exposed to an achromatic homogeneous field. In the binocular rivalry group, the right eye was exposed to an achromatic "jazzy" pattern of varying contrastive shapes. The strength of aftereffect tested with the left eye depended upon the duration of the inspection phase, regardless of the subject's failure to see the colored grating during binocular suppression by the jazzy pattern. These data raise the issue of whether there exists a wavelength-sensitive stage prior to the stage at which a rivalry-sensitive conscious percept is generated. Moreover, if such a stage does exist, then why are its adaptational mechanisms still active during the suppression phase of rivalry?

In Experiment II (Figure 19B), binocular differentcolor and like-color conditions were studied. In both conditions, the left eye was exposed to alternating magenta verticals and green horizontals. In the like-color condition, the right eye was exposed to homogeneous colored fields whose color matched that of the stripes presented simultaneously to the left eye. In the different-color condition, the right eye was exposed to homogeneous colored fields whose color was opposed to that of the stripes presented simultaneously to the right eye. The right eye was then tested with achromatic striped patterns. In the like-color condition, normal aftereffects were observed; for example, a green aftereffect to achromatic vertical lines was observed if the left eye inspected magenta verticals while the right eye inspected a homogeneous magenta field. In the different-color condition, reversed aftereffects were observed; for example, a magenta aftereffect to achromatic vertical lines was observed if the left eye inspected magenta verticals while the right eye inspected a homogeneous green field. The strength and persistence of this reversed aftereffect were not as great as those of normal aftereffects.

White et al. (1978) compared this result with MacKay and MacKay's $(1973,1975)$ reports that when an achro-

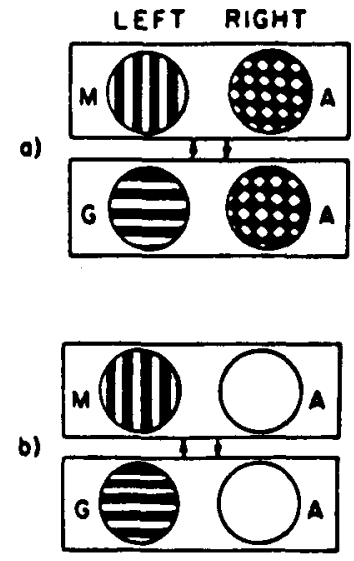

(A)

a)

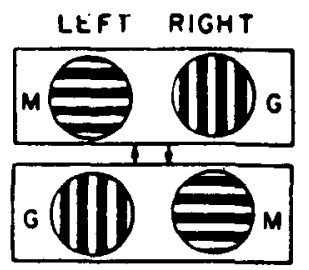

b)

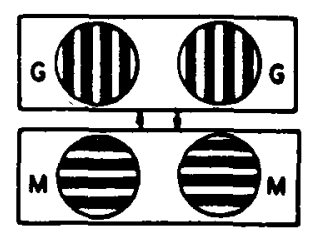

(C) o)

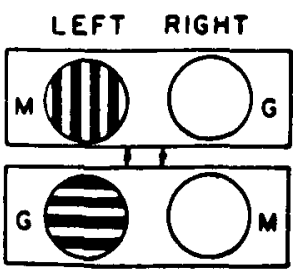

b)

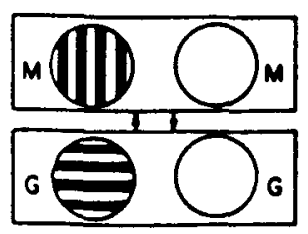

(B)

a)

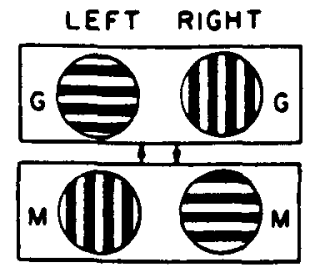

b)

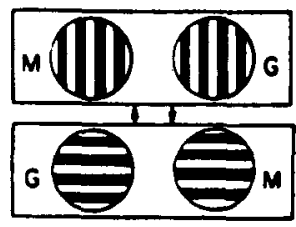

(D)
Figure 19. Stimuli used by White, Petry, Riggs, and Miller (1978): (A) Inspection conditions of their Experiment I: In either condition, the left eye views alternating magenta (M) and green (G) gratings. In Condition a (rivalry), the right eye views an achromatic "janzy" pattern. In Condition b (no rivalry), the right eye views an achromatic homogeneous field. (B) Inspection conditions of Brperiment II: (a) Different-color condition-Presentation of a magenta (M) vertical grating to the left eye poired with a homogeneous green (G) field to the right eye, alternated with a green grating paired with a homogeneous magenta field. (b) Lke-color condition-Magenta vertical grating paired with a homogeneous magenta field alternates with a green grating paired with a homogeneons green field. (C) Inspection conditions of Experiment III: (a) Dinerent-color/differentorientation condition-Presentation of a mogenta (M) hortwontal grating to the left eye paired with a green (G) vertical grating to the right eye alternated with a green vertical grating paired with a magenta horizontal grating. (b) Like-color/hike-orientation condition-Green vertical gratings allernated with megenta horizontal gratings. (D) Inspection conditions of Experiment IV: (a) Likecolor/different-orientation condition. Presentation of a green (G) horizontal grating to the left eye paired with a green vertical grating to the right eye, alternated with a magenta $(M)$ vertical grating paired with a magenta horizontal grating. (b) Different-color/likeorientation condition-Magenta vertical grating paired with a green vertical grating alternated with a green horizontal grating paired with a magenta horizontal grating. From "Binocular Interactions During Establishment of McCollough Effects" by K. D. White, H. M. Petry, L. A. Riggs, and J. Miller, 1978, Vision Research, 18, 12011215. Copyright 1978 by Pergamon Press. Reprinted by permission. 
matic grating was presented to one eye and a homogeneous colored field was presented to the other eye, testing of the former eye led to a reversed aftereffect whereas testing of the latter eye led to a normal aftereffect. White et al. (1978) discuss some of the problems that other investigators have had in replicating these results. Savoy (personal communication, 1985) has replicated these data.

The results of Experiment II raise the issue of how a homogeneous colored field can energize aftereffects that are sensitive to orientations presented through the other eye, and thus are strongly influenced by binocular rivalry, unlike the aftereffects found in Experiment I. Also, why do these binocularly mediated orientations generate aftereffects that are contingent upon the color of the homogeneous colored field rather than upon the color of the inspection grating? In other words, why does the orientation transfer binocularly but not the color? On the other hand, why, in studies that use achromatic gratings to one eye, such as MacKay and MacKay's (1973, 1975), is there a tendency for the opposite color to transfer binocularly?

These issues are dramatized by Experiment III (Figure 19C), in which a different-color/differentorientation condition is compared with a like-color/likeorientation condition. Subjects were tested with monocular achromatic gratings as well as with binocular achromatic gratings. The binocular test score was greater than each of the monocular test scores following inspection in the like-color/like-orientation condition. In contrast, the monocular scores were both greater than the binocular score following the different-color/different-orientation inspection condition. These data illustrate that rivalry both of orientations and of colors needs to be taken into account in order to analyze binocular transfer effects.

Experiment IV (Figure 19D) provided more information about this interaction by also considering likecolor/different-orientation and different-color/likeorientation conditions. Monocularly tested aftereffects were weakest after inspection in the like-color/differentorientation condition.

My discussion builds upon previous analyses (Grossberg, 1980, 1983b) in which I showed how interactions between an adaptive filter and a double-opponent gated dipole field could generate long-term color-contingent aftereffects. These analyses linked the existence of McCollough-type effects to general mechanisms of visual information processing before the BC System and FC System were discovered. I now outline how these general mechanisms can explain all of the above data properties when they are embedded within the network in Figure 1 that is introduced herein.

Adaptational properties consistent with McCollougheffect data are generated within the FC System pathways from the monocular preprocessing stages to the monocular syncytia and from the monocular syncytia to the binocular syncytia (Figure 1). The total network architecture modulates the processing within these FC System pathways. To understand how these properties arise, first one needs to know what the adaptational mechanisms are and then one needs to know how the total network modulates these mechanisms.

These mechanisms have been described elsewhere and are the same mechanisms that have been used to discuss negative afterimages in this article: "Pattern-contingent colored aftereffects can also be generated in a dipole field. Suppose that a prescribed field of feature detectors is color coded. Let the on-cells be maximally turned on by red light and the off-cells be maximally turned on by green light for definiteness. Then white light will excite both on-cells and off-cells; that is, white light acts like an adaptation level in this situation. Suppose that a red input whose features are extracted by the field is turned on long enough to substantially deplete its transmitter. What happens if a white light replaces the red input on test trials? The depletion caused by the prior red input now causes the white adaptation level to generate a larger gated signal to the green channel, so a green pattern-contingent aftereffect will be generated"' (Grossberg, 1980, p. 22).

How can the long duration of McCollough aftereffects be explained? Before the BC System and FC System were discovered, two types of mechanisms were suggested to explain how the persistence of these aftereffects could extend long beyond the time of recovery from the transmitter habituation process. One of these mechanisms used the contrast-enhancement and short-term-memory properties of recurrent on-center off-surround networks (Grossberg, 1980, p. 22). These properties are now relegated to the CC loop of the BC System. The other mechanism is advocated here for the FC System: "The imbalance due to asymmetric habituation can also be encoded in the LTM traces due to the property that a decrease in postsynaptic STM activity can cause a decrease in LTM strength. Such an LTM change is not a case of associative learning due to CS-UCS (conditioned stimulus, unconditioned stimulus) contiguity. Rather, it is a case of retuning feature detectors to their trigger input patterns. Thus habituation within a nonclassical opponent process interacting with a non-Hebbian adaptive filter [cf. Section 23] can explain McCollough effect properties as manifestations of a basic processing design"' (Grossberg, 1983b, p. 680).

These general mechanisms are specialized within the FC System as follows. The pathways from the monocular preprocessing stages to the monocular syncytia and from the monocular syncytia to the binocular syncytia are assumed to contain non-Hebbian LTM traces that multiplicatively gate the signals in their respective pathways (cf. Section 23). The collection of all the adaptive pathways from a monocular preprocessing stage to a monocular syncytium comprises an adaptive filter. So too does the collection of all the adaptive pathways from a monocular syncytium to a binocular syncytium. These adaptive filters work together with double-opponent networks to process output signals on their way to the next syncytial stage. The primary function of the adaptive filters is to maintain selective contacts between FC System cells that code the same positions and colors; thus red cells map into red cells, green cells map into green cells, and so 
on, at corresponding positions due to the adaptive tuning carried out by the LTM traces.

Since each double-opponent network is a gated dipole field, its pathways contain habituative transmitters that multiplicatively gate the signals carried by the pathway. Thus, the adaptive filter pathways are doubly gated: first, by habituative transmitters that regulate the opponent balance between coupled ON and OFF channels, notably their antagonistic rebounds; and second, by non-Hebbian LTM traces which slowly track the habituatively gated signals and doubly gate these signals on their way to their target syncytium.

Using this combination of double-opponent and nonHebbian adaptive filter processing, I now show how the global anatomy of BC System and FC System interactions enables different scenic inputs to activate combinations of FC System pathways that are sensitive to the orientations of scenic contrasts. Since each FC System pathway is also selective for color (red $\rightarrow$ red, green $\rightarrow$ green, etc.), a pathway that is activated by a particular scenic orientation automatically correlates that orientation with the color system in which the pathway is found. This type of correlated adaptation is not classical conditioning, in agreement with previous experimental work on the McCollough effect (McCarter \& Silver, 1977; Savoy, 1984; Skowbo, 1984; Skowbo \& Forster, 1983; Skowbo \& White, 1983).

Some McCollough effect data can be analyzed using properties of the adaptive reactions within individual FC System pathways, without regard to the spatial patterning of these pathways. For example, persistent input to an FC System pathway can habituate its transmitter gate. The total amount of habituation covaries with the duration of the input, since the total habituation is a cumulative process. This property is consistent with the data of Skowbo and White (1983), who showed that the strength of the McCollough effect depends upon the duration, not the number, of exposures. If the input to a pathway persists long enough for a cumulative habituation to occur within its transmitter gate, then-because it obeys a nonHebbian associative law-the slowly varying LTM trace along the pathway senses the persistent decrease in its transmitter-gated signal and also begins to decrease (Figure 20). Thus, the LTM change is also sensitive to the duration of the signal. This LTM change can, however, endure long after the transmitter habituation recovers.

The opponent organization of the FC System pathways clarifies how an achromatic input can elicit a long-term opponent-color aftereffect, yet how repeated presentation of the achromatic input can extinguish the aftereffect (Savoy, 1984; Skowbo et al., 1975). Suppose, for definiteness, that prior input to an $\mathrm{ON}$ pathway has previously caused substantial transmitter habituation and decrease of the pathway's LTM trace. Suppose that the transmitter has already recovered from its habituation but that the LTM trace has not been retrained (Figure 21). Let an achromatic scenic edge generate equal signals within the
ON CHANNEL

OFF CHANNEL

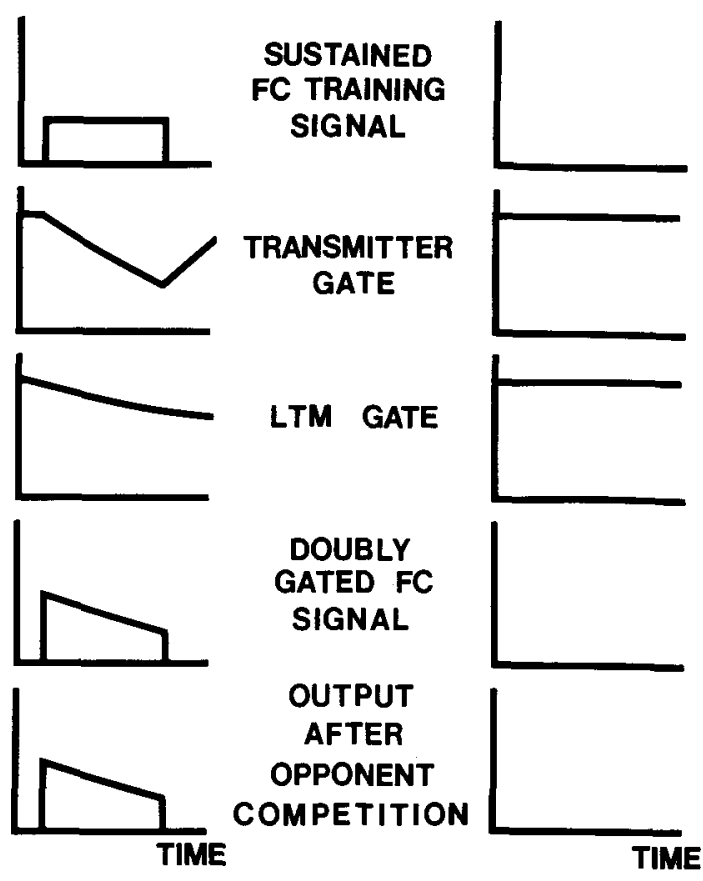

Figure 20. Interaction between sustained FC signal, habituating transmitter gate, long-term-memory (LTM) trace, and opponent competition between $O N$ and OFF channels: A sustained FC training signal to the $O N$ channel causes a cumulative habituation of its transmitter. The LTM trace slowly tracks the transmitter-gated FC signal-(FC signal) times (transmitter)-and also decreases. The LTM trace remains small even after the transmitter recovers. The net signal from this pathway equals (FC signal) times (transmitter) times (LTM trace). Net signals from the ON and OFF channels compete subtractively to generate outputs to the next FC stage.

ON pathway and its corresponding OFF pathway. Then, the smaller LTM-trace gates the signal in the previously habituated pathway. Consequently, the opponent OFF pathway delivers a larger LTM-gated input, and a longterm opponent-color aftereffect is generated. Suppose that this white input is persistently presented. Then, equal amounts of cumulative transmitter habituation are caused in the opponent channels and their LTM traces equalize by tracking these equalized transmitter-gated signals. In this way, a white input trains equal LTM-gated signals within the opponent channels and the aftereffect is actively extinguished.

I now discuss how different combinations of scenic images can selectively activate different subsets of FC System pathways in a manner that mimmicks monocular and binocular McCollough effect data. The core issue is, of course, why the McCollough effect is orientationsensitive. Remarkably, orientation-sensitive aftereffects can be generated even if none of the FC System cells possesses an oriented receptive field. In the subsequent discussion, I separate the properties of orientation-sensitive aftereffects that can arise from nonoriented FC System cells from the properties of orientation-sensitive after- 
ON CHANNEL
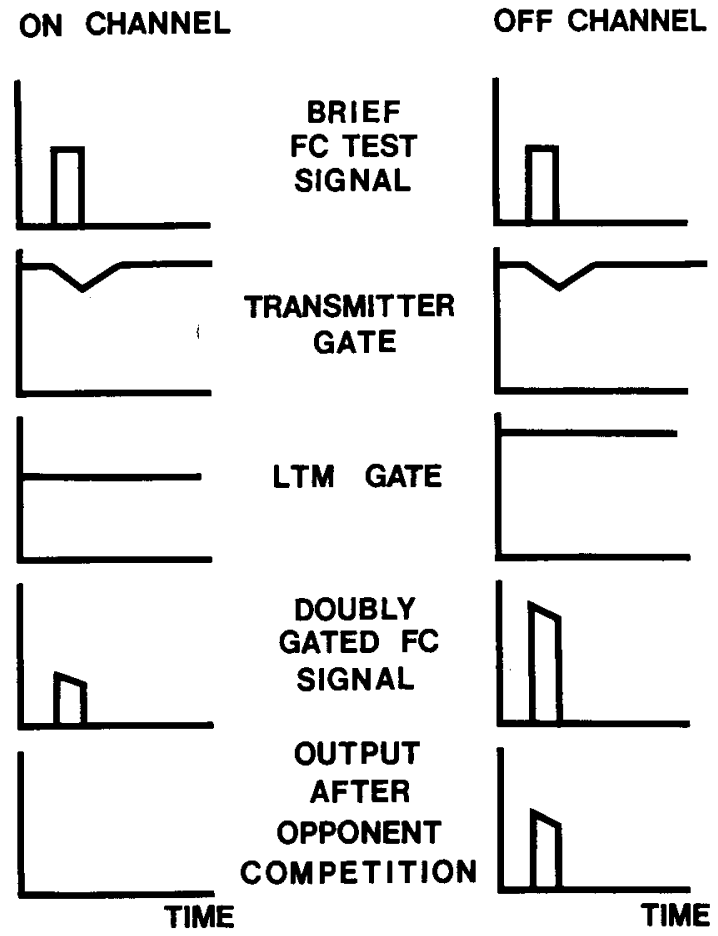

Figure 21. Generation of a long-term color aftereffect: After the transmitter in Figure 20 recovers, the LTM trace in the ON channel can remain small. A brief test signal that equally excites both opponent channels (e.g., white) can cause minor, but equal, habituation of the transmitters. However, gating of the signal by the LTM traces generates a larger net signal in the OFF channel than the ON channel. After opponent competition, the OFF channel generates an output $F C$ signal.

effects that can arise from oriented FC System cells. This separation is conceptually necessary because the relative proportion of nonoriented-to-oriented FC System cells may vary across species, or oriented cells may be totally absent, without our denying the existence of orientationsensitive aftereffects.

Oriented receptive fields can, in principle, exist within the FC System at any of several stages. For example, a double-opponent gated dipole field transforms the activities of a monocular syncytium to output signals to a binocular syncytium (Section 26). This gated dipole field may, in principle, possess orientation-sensitive cells. Evidence for this possibility could, for example, include the discovery of orientation-sensitive cells that receive inputs from area 17 blobs and output to color-coded area 18 stripes. Such orientational tuning could facilitate the binocular matching of monocular inputs from pairs of monocular syncytia before the matched inputs could activate the binocular filling-in process (Section 18). If the output signals from the monocular syncytia are both orientationally tuned and chromatically tuned, then the habituative and LTM-trace changes described above would all depend upon both the orientation and the color of the stimuli, yet would not be due to classical conditioning of orientation to color.
Oriented receptive fields can also exist in the pathways from the monocular preprocessing stages to the monocular syncytia. Oriented cells are, for example, known to exist in cat retinal ganglion cells (Leventhal \& Schall, 1983; Levick \& Thibos, 1982), cat lateral geniculate cells (Daniels, Norman, \& Pettigrew, 1977; Vidyasagar \& Urbas, 1982), and monkey lateral geniculate cells (Lee, Creutzfeldt, \& Elepfandt, 1979).

Orientation-sensitive responses within the FC System may be generated in either of two ways if all FC System cells possess unoriented receptive fields. The first mechanism can influence only pathways from the monocular syncytia to the binocular syncytia. The second mechanism can influence pathways from the monocular preprocessing stages to the monocular syncytia and from the monocular syncytia to the binocular syncytia. The first mechanism is based upon the fact that the BC System is organized into ocular dominance columns and orientationally tuned hypercolumns (Section 22 and Figure 10). Different orientations corresponding to a given retinal position are coded at slightly different hypercolumn positions. Different orientations thus send FIG signals to slightly different cells within the monocular syncytium. These cells can, in turn, activate slightly different FC System pathways to the binocular syncytium. Consequently, the orientational tuning within the BC System may indirectly cause orientationally sensitive retuning of the adaptive filter between the monocular and binocular syncytia by causing statistically significant differences in the spatial patterning of FC System pathways that are activated by different orientations. This type of mechanism can also operate when BC System cells are selectively activated by oriented edges moving in prescribed directions.

The second mechanism cannot cause orientationally sensitive adaptation in response to moving scenic edges. It is effective only when static images are presented in a prescribed spatial relation to a fixation point. This mechanism uses the property that orientationally sensitive FIG signals can group the color signals from adapted and nonadapted pathways into oriented regions for featural filling-in. Figure 22 schematizes regions in which red, or magenta (M), green (G), or both (A) adaptations occur within the FC System due to alternate monocular presentations of red verticals and green horizontals. When achromatic verticals are presented, they cause vertical FIG signals to divide the syncytia into vertical filling-in domains. Due to adaptation of the red-coded FC System pathways, the red filling-in is weaker than the green filling-in. After opponent processing occurs, a green vertical aftereffect can be generated. For the same reason, a red horizontal aftereffect can be generated. The achromatic (A) regions do not contribute to the aftereffects, because their pathways are equally adapted in both directions (assuming all FC System cells are nonoriented). The FIG-induced organization of the syncytia into vertical or horizontal domains enables opposite chromatic aftereffects to fill in over the chromatically neutral ( $A$ and $\mathrm{Bl}$ ) regions when the orientation of the FIG signals is reversed. Be- 


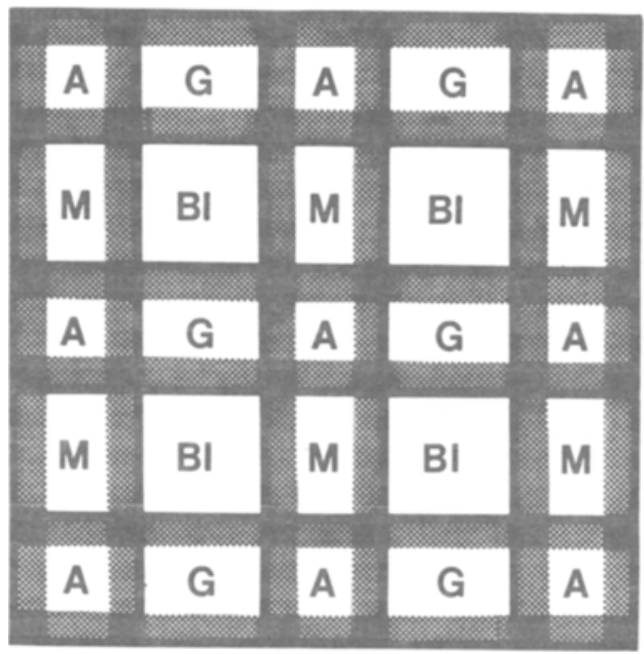

Figure 22. Alternate viewing of green (G) and black horizontal bars and magenta (M) and black vertical bars can cause a lattice of chromatically adapted regions to emerge: $R, G$, achromatic or equally adapted (A), and black (B). The stippled regions indicate positions where chromatic adaptation occurs. Horizontal hatched areas adapt to green, and vertical hatched areas adapt to magenta. Viewing of achromatic horizontal test bars can generate boundary contours that enable $G$ and $A$ regions to fill in over each white bar within the $\mathbf{G}$ syncytium, thereby generating an uninterrupted band of green monocular syncytial activity. In addition, the white input enables $M$ and $A$ regions to fill in over each white bar within the $M$ syncytium. Because the green FC pathways are adapted, after opponent processing takes place, horizontal bands of magenta FC output signals are generated. A similar analysis shows how vertical bands of green FC output signals are generated in response to vertical achromatic white and black bars.

cause of these mechanisms, opposite monocular aftereffects can be trained in the same individual using, say, green verticals and red horizontals, because these stimuli preferentially activate, and therefore adapt, positionally disjoint subsets of pathways between different syncytia, whether or not any FC System cells have oriented receptive fields.

The acquisition of the McCollough effect does not transfer interocularly (McCollough, 1965; Murch, 1972; White et al., 1978), and extinction of the McCollough effect does not transfer interocularly (Savoy, 1984), in these networks for the following reason: Monocular presentation of oriented chromatic or achromatic stimuli activates the BC System, which, in turn, sends FIG signals to both the left and the right monocular syncytia. On the other hand, only the eye that receives the monocular stimulus receives FC System inputs to its monocular syncytia, and only these monocular syncytia can generate FC output signals to the binocular syncytia. The monocular preprocessing stage and monocular syncytia of the unstimulated eye cannot activate their FC System output pathways, hence cannot undergo the adaptational LTM changes that are contingent upon sustained activation of these pathways.

A similar argument suggests how a single individual can acquire opposite monocular and binocular McCol- lough effects in response to cyclic presentation for $30 \mathrm{~min}$ of the following sequence of stimuli: red vertical, binocular; blue vertical, right eye; red horizontal, right eye; blue horizontal, binocular; red horizontal, left eye; blue vertical, left eye (Vidyasagar, 1976). The primary property needed to explain this result is that binocular viewing of these images excites a different spatial distribution of binocular FIG pathways and monocular-to-binocular FC System pathways than does monocular viewing. This property is one of the basic means whereby double images are suppressed and a coherent representation of formand-color-in-depth is generated. The multiple spatial scales of the BC System typically react differently to monocular versus binocular viewing of simple pairs of images (Sections 2-9 and 24). In addition, binocular mismatch of pairs of monocular FC signals from the monocular syncytia can prevent activation of cells that would have responded to just one monocular syncytium (Section 25). Since adaptive tuning of an LTM trace is controlled by activation of its pathway's postsynaptic cell, certain pathways that could have adapted in response to monocular viewing may not adapt to binocular viewing.

\section{Interocular Transfer Properties of McCollough Effect}

The data from Experiment I of the White et al. (1978) study are consistent with the fact that transmitter habituation and LTM changes can occur continuously in the pathways from the monocular preprocessing stages to the monocular syncytia, even when the percept from the corresponding eye is suppressed due to binocular rivalry. Binocular rivalry influences the FC System only from the monocular syncytia onward. Thus, comparison of the amount of adaptation in the rivalry and no-rivalry conditions when both conditions are equated for the time that the percept is seen should yield higher levels of adaptation in the rivalry condition, as found in the data. A number of subtle interactions can also occur due to rivalry at the binocular syncytia. The properties of these interactions are best seen through consideration of Experiments II-IV.

The data from Experiment II of the White et al. (1978) study show that, although the McCollough effect does not transfer interocularly under monocular acquisition conditions, it does transfer interocularly if the nonadapted eye is presented with a homogeneous colored field during adaptation. This type of interocular transfer can be explained by adaptation that occurs subsequent to the monocular syncytia, as follows. Although transmitter habituation and LTM adaptation can occur in the adaptive filter from the right monocular preprocessing stage to the monocular syncytia, due to the homogeneity of the right-eye training image, such adaptation cannot be sensitive to the orientation of an achromatic test grating. Moreover, alternate presentation of magenta and green homogeneous training images to the right eye tend to cause equal levels of adaptation in the corresponding opponent color systems. These balanced adaptations would tend to 
cancel any aftereffect. The interocular transfer effects reported in Experiment II must therefore be traced to adaptation effects subsequent to the monocular syncytia.

A homogeneous colored field does not, however, contain interior edges. If spatially discontinuous inputs are needed to elicit FC outputs from a monocular syncytium, yet there are no interior edges in a homogeneous field with which to generate spatially discontinuous inputs, why does a homogeneous colored field have any effect whatsoever on interocular transfer?

A possible explanation of these data arises from consideration of how a FIG signal restricts filling-in within a monocular syncytium. As noted in Section 24 of Grossberg (1987b), a FIG signal acts as an inhibitory gating signal that causes an increase in the membrane resistance of its target syncytial cells. Thus, a FIG signal also acts as an inhibitory FC input to its target syncytial cells. When no other inputs reach the monocular syncytia, this inhibitory signal cannot create excitatory output signals from the monocular syncytia. When excitatory inputs do activate the monocular syncytium, a FIG signal can cause a spatial discontinuity in the syncytium's filled-in activity due to the fact that it can trap its own inhibitory signals within the cells whose membrane resistance it has increased. The size of this spatial discontinuity may increase, albeit nonlinearly, with the luminance of the homogeneous colored field.

We have now reached a crucial point in the argument. If homogeneous colored fields can generate FC outputs to the binocular syncytium, then why cannot all mismatched monocular images do so? Why does not the entire theoretical structure for preventing double images collapse? Why is the whole argument about suppression during binocular rivalry not invalid? There is a "safe" answer that cannot explain the intraocular transfer data, and there is a more interesting answer that can.

The safe answer posits that the spatial scale of a FIG is too fine to be detected by the on-center off-surround interactions of a double-opponent gated dipole field. A spatial discontinuity in filled-in syncytial activities must occur across a wider domain than the thickness of a FIG in order to generate FC output signals. Hence, FIG discontinuities surrounded by equal filled-in syncytial activities cannot generate FC output signals, and thus do not influence perception.

The interesting answer builds from the observation that homogeneous colored fields are essentially the only colored images that do not excite any nontrivial opponent color reactions. Given essentially any colored inhomogeneous image, an ON input to one region is associated with an opponent OFF input to a contiguous region. After filling-in takes place, the ON and OFF outputs at every position of the monocular syncytium, including positions along the FIG, tend to inhibit each other if the FIG does not spatially interpolate the ON and OFF inputs. Thus, the previous explanations hold for colored images that contain contrastive contours, despite the fact that a homogeneous colored image can support interocular transfer of the McCollough effect.

It is nonetheless probable that a FIG signal to a homogeneously activated monocular syncytium cannot generate an FC output signal that is as large as one generated from an inhomogeneously activated monocular syncytium. The bandwidth of a FIG signal may be detectable by the on-center off-surround gated dipole field, but may be too narrow to generate optimal output signals to the binocular syncytium. Thus, the aftereffects generated by the right eye may involve fewer pathways, whose transmitters habituate less and whose LTM traces adapt less. The interocular transfer aftereffects reported by White et al. (1978) were, in fact, weak and less persistent than the aftereffects generated through the left eye.

It remains to say why the right-eye aftereffect was in the color opponent to the color of the homogeneous test field. In the like-color condition, this property can be explained using the usual mechanism: When the right eye inspected a homogeneous magenta field while the left eye inspected magenta verticals, orientationally tagged red cells inputting to the binocular syncytium were binocularly activated. The active input pathways underwent the usual habituation and LTM tuning, therefore favoring a green aftereffect in response to achromatic verticals. In the different-color condition, the aftereffect can be traced to binocular rivalry within the double-opponent network that inputs to the binocular syncytium: When the right eye inspected a homogeneous green field while the left eye inspected magenta verticals, both green and red orientationally tagged pathways to the binocular syncytia would be activated and would compete. The right-eye green pathways would habituate and retune their LTM traces when these pathways won the opponent competition, thereby generating a magenta aftereffect in response to achromatic verticals.

The tendency to get reversed aftereffects, as reported by MacKay and MacKay $(1973,1975)$ and Savoy (personal communication, 1985), can be explained as follows. This explanation ignores the sources of binocular suppression that may weaken this tendency. Suppose that an achromatic grating is presented to the left eye and a homogeneous magenta field is presented to the right eye. The achromatic grating generates binocular FIGs which enable FC output signals to be generated from red-coded monocular syncytia of the right eye. These signals habituate the transmitter gates in the active red-coded righteye pathways and activate the binocular red-coded cells that input into the binocular red-coded syncytia. The achromatic left-eye input tends to equally activate both the red-coded and the green-coded pathways from the left monocular syncytia to the binocular syncytia. The transmitters in these pathways thus tend to undergo an equal amount of habituation. On the other hand, the red-coded binocular cells are more active than the green-coded binocular cells due to the summation of inputs from left eye and right eye at the red-coded cells. Consequently, 
the LTM traces in the red-coded left-eye pathways to these binocular cells tend to become larger than the LTM traces in the green-coded active left-eye pathways. During test trials, an achromatic grating to the right eye tends to generate a green aftereffect, whereas an achromatic grating to the left eye tends to generate a red aftereffect.

In Experiments III and IV, the pathways from the monocular preprocessing stages to the monocular syncytia experience similar levels of adaptation in all experimental conditions. Adaptation within these monocular pathways determines an adaptational baseline that is augmented to different degrees in the four experimental conditions by the adaptation that occurs in the pathways from the monocular syncytia to the binocular syncytia. Thus, my explanations of these data depend upon the hierarchical organization of FC System pathways from the monocular preprocessing stages to the binocular syncytia, which enables a cumulative adaptation effect to be registered at the binocular syncytia.

In the like-color/like-orientation condition of Experiment III (Figure 19Cb), left-eye and right-eye inputs can cooperate both at binocular BC System cells and at binocular FC System cells that input to the binocular syncytia. In contrast, the different-color/different-orientation condition (Figure 19Ca) causes rivalry to occur both within the BC System and at the binocular FC System cells. Consequently, binocular test gratings lead to stronger aftereffects than do monocular test gratings in the likecolor/like-orientation condition, whereas the opposite is true in the different-color/different-orientation condition.

To explain the main finding of Experiment IV that monocular testing of the like-color/different-orientation condition (Figure 19Da) yields the weakest aftereffect, a finer analysis of the different-color/different-orientation of Experiment III is needed. Consider a time when horizontal FIG signals are activated by magenta horizontals to the left eye. The FIG signals activate red-coded horizontally sensitive pathways from the left monocular syncytia to the binocular syncytia. The FIG signals also divide the right monocular syncytia into horizontal strips. Due to the narrow bandwidth of the FIG signals, weak activation of green-coded horizontally sensitive pathways from the right monocular syncytia to the binocular syncytia occurs. Rivalry between these opponent-color pathways can occur at the binocular syncytia, leading to both red and green adaptation in horizontally sensitive pathways. These opponent adaptations lead to a weakened net adaptive effect, but the red-horizontal correlation may nonetheless tend to be stronger than the green-horizontal correlation. For a similar reason, the green-vertical correlation tends to be stronger than the red-vertical correlation.

In contrast, consider a time during the likecolor/different-orientation condition (Figure 19Da) when horizontal FIG signals are activated by green horizontals to the left eye. The FIG signals activate green-coded horizontally sensitive pathways from both the left and the right monocular syncytia. There is no color rivalry at the binocular syncytia. Hence, the green-coded horizontally sensitive pathways from the right eye can adapt more in this condition than in the comparable differentcolor/different-orientation condition. Under achromatic monocular right-eye testing, these pathways can therefore more effectively inhibit the red-coded horizontally sensitive pathways that adapted in response to presentation of magenta horizontal stripes to the right eye. In summary, in the like-color/different-orientation condition, color rivalry at the binocular syncytium cannot spare the correlation between orientation and color that is endangered in pathways from the monocular syncytia to the binocular syncytia by the orientation rivalry taking place within the BC System.

A variety of other data concerning McCollough-type aftereffects can be analyzed in terms of such interactions. For example, Broerse and Crassini (1986) have shown that McCollough-type adaptation can be used to render ambiguous displays, such as the diamond-rectangles figure, unambiguous. These data can be analyzed, as in the above discussion, by a combination of BC System and FC System rivalry and adaptational mechanisms.

\section{Concluding Remarks}

This article introduces a perceptual theory that explains in a principled and unified way a large body of data about 3-D form, color, and brightness perception. Many of these data have received no previous explanation, let alone an explanation as part of a general perceptual theory. The article also describes a neural architecture whose interactive properties generate the theory's explanations of perceptual data. This architecture is well enough characterized to test it in multiple ways through experiments on striate and prestriate visual cortex. Mathematical and computer analyses of different pieces of this architecture are developed in a number of other articles (Cohen \& Grossberg, 1984a, 1984b, 1986, in press; Grossberg \& Marshall, 1987; Grossberg \& Mingolla, 1985a, 1985b, 1986, 1987), and further quantitative analyses are presently under way.

Underlying these detailed mechanisms and data explanations are a number of new design principles and concepts about visual perception. Concepts such as monocular syncytium and binocular syncytium, gated dipole field, filling-in generator and filling-in barrier, $O C$ filter and CC loop, orientation field, masking field, orthogonal end cut, and non-Hebbian adaptive filter can guide the theoretical development of a more complete visual theory. Details concerning anatomical connections may, for example, be different across species without requiring such concepts to be abandoned. Design principles such as boundary-feature tradeoff and the several new uncertainty principles that govern the organization of successive processing stages within the BC System and the FC System may also be used as a foundation for building a more complete theory. Another general foundational theme is that mechanisms of boundary segmentation and featural filling-in and of stereopsis and boundary segmentation can best be understood through an analysis of their interactions 
rather than as independent modules. This approach led to the discovery that double-opponent networks of unoriented color cells can compute oriented form-sensitive properties when they interact with $\mathrm{BC}$ signals, and that, paradoxically, this particular type of orientational sensitivity helps to explain the nonspecific suppression that occurs during binocular rivalry. The explanations of why the BC System becomes binocular at an earlier processing stage than the FC System, of how the CC loop generates a globally consistent, sharp, yet deformable, binocular segmentation from the multiplexed signals of binocular complex cells, and of how perceptually invisible BCs can influence object recognition are among the other general results that promise to persist in a more complete theory.

Even as it stands, however, the theory suggests explanations of an unusually large perceptual and neural data base, and provides tools for explaining a large body of perceptual data that $I$ have not considered in detail, but for which a direct attack is now clearly indicated-for example, data about metacontrast and stereoscopic capture. The theory has also already articulated qualitatively new mathematical designs for parallel machines capable of rapidly synthesizing coherent and informative global visual representations of the external world. It remains to be seen just how far these ideas can carry us toward a complete perceptual and neural theory of form-andcolor-in-depth and a new generation of real-time contextsensitive massively parallel vision machines.

\section{REFERENCES}

Beck, J., Prazdny, K., \& Ivry, R. (1984). The perception of transparency with achromatic colors. Perception \& Psychophysics, 35 , $407-422$.

Beverley, K. I., Regan, D. (1979). Separable aftereffects of changing-size and motion-in-depth: Different neural mechanisms. Vision Research, 19, 727-732.

Bienenstock, E. L., Cooper, L. N., \& Munro, P. W. (1982). Theory for the development of neuron selectivity: Orientation specificity and binocular interaction in visual cortex. Jourmal of Neuroscience, 2, 32-48.

BLAKE, R. (1977). Threshold conditions for binocular rivalry. Journal of Experimental Psychology: Human Perception \& Performance, 3 , 251-257.

BlaKe, R. CAMISA, J. (1979). On the inhibitory nature of binocular rivalry suppression. Joumal of Experimental Psychology: Human Perception \& Performance, 5, 315-323.

BLAKE, R., \& Fox, R. (1974). Binocular rivalry suppression: Insensitive to spatial frequency and orientation change. Vision Research, 14, 687-692.

BLAKE, R., \& LEMA, S. A. (1978). Inhibitory effect of binocular rivalry suppression is independent of orientation. Vision Research, 18, 541-544.

Blake, R., Sloane, M., \& Fox, R. (1981). Further developments in binocular summation. Perception \& Psychophysics, 30, 266-276.

Blakemore, C., CoOper, G. F. (1970). Development of the brain depends on the visual environment. Nature, 224, 477-478.

BraAstad, B. O., HegGelund, P. (1985). Development of spatial receptive-field organization and orientation selectivity in kitten striate cortex. Journal of Neurophysiology, 53, 1158-1178.

Brokrse, J., \& Crassini, B. (1986). Making ambiguous displays unambiguous: The influence of real colors and colored aftereffects on perceptual alternation. Perception \& Psychophysics, 39, 105-116.
Carpenter, G. A., \& Grossberg, S. (1983). A neural theory of circadian thythms: The gated pacemaker. Biological Cybernetics, 48, 35-59.

Carpenter, G. A., \& Grossberg, S. (1984). A neural theory of circadian thythms: Aschoff's rule in diurnal and nocturnal mammals. American Journal of Physiology, 247, R1067-R1082.

Carpenter, G. A., GRossberg, S. (1985). A neural theory of circadian rhythms: Split rhythms, after-effects, and motivational interactions. Journal of Theoretical Biology, 113, 163-223.

Cogan, A. I. (1982). Monocular sensitivity during binocular viewing. Vision Research, 22, 1-16.

Cogan, A. I., Silverman, G., \& Sekuler, R. (1982). Binocular summation in detection of contrast flashes. Perception \& Psychophysics, 31, 330-338.

COHEN, M. A., \& Grossberg, S. (1984a). Neural dynamics of brightness perception: Features, boundaries, diffusion, and resonance. $P e r$ ception \& Psychophysics, 36, 428-456.

Cohen, M. A., * Grossberg, S. (1984b). Some global properties of binocular resonances: Disparity matching, filling-in, and figure-ground synthesis. In P. Dodwell \& T. Caelli (Eds.), Figural synthesis. Hillsdale, NJ: Erlbaum.

Cohen, M. A., \& GossBerg, S. (1986). Neural dynamics of speech and language coding: Developmental programs, perceptual grouping, and competition for short term memory. Human Neurobiology, 5, 1-22.

CoHEN, M. A., Grossberg, S. (in press). Masking fields: A massively parallel neural architecture for learning, recognizing, and predicting multiple groupings of patterned data. Applied Optics.

CURTIS, D. W., \& RuLE, S. J. (1980). Fechner's paradox reflects a nonmonotone relation between binocular brightness and luminance. Perception \& Psychophysics, 27, 263-266.

DANiel, P. M., \& WhitTeridge, D. (1961). The representation of the visual field in the cerebral cortex of monkeys. Joumal of Physiology, 159, 302-321.

Daniels, J. D., Norman, J. L., \& Pettigrew, J. D. (1977). Biases for oriented moving bars in lateral geniculate nucleus neurons of normal and stripe-reared cats. Experimental Brain Research, 29, 155-172.

Daugman, J. G. (1980). Two-dimensional spectral analysis of cortical receptive field profiles. Vision Research, 20, 847-856.

Desimone, R., Schein, S. J., Moran, J., \& Ungerleider, L. G. (1985). Contour, color, and shape analysis beyond the striate cortex. Vision Research, 25, 441-452.

DEV, P. (1975). Perception of depth surfaces in random-dot stereograms: A neural model. International Journal of Man-Machine Studies, 7, 511-528.

De Valois, R. L., Albrecht, D. G., \& Thorell, L. G. (1982). Spatial frequency selectivity of cells in macaque visual cortex. Vision Research, 22, 545-559.

Doshrr, B., SPERLing, G., \& WuRst, S. (1986). Tradeoffs between stereopsis and proximity luminance covariance as determinants of perceived 3D structure. Vision Research, 26, 973-990.

Dow, B. M., SNyder, A. Z., VAutin, R. G., \& BAuer, R. (1981). Magnification factor and receptive field size in foveal striate cortex of the monkey. Experimental Brain Research, 44, 213-228.

EARLE, D. C. (1985). Perception of Glass pattern structure with stereopsis. Perception, 14, 545-552.

EgusA, H. (1983). Effects of brightness, hue, and saturation on perceived depth between adjacent regions in the visual field. Perception, 12, 167-175.

EMMERT, E. (1881). Grössenverhaltnisse der Nachbilder. Klinische Monatsblaetter für Augenheillunde, 19, 443-450.

Foley, J. M. (1980). Binocular distance perception. Psychological Review, 87, 411-434.

Fox, R., \& CHECK, R. (1972). Independence between binocular rivalry suppression duration and magnitude of suppression. Joumal of $E x-$ perimental Psychology, 93, 283-289.

FréGNAC, Y., \& IMBERT, M. (1978). Early development of visual cortical cells in normal and dark-reared kittens: Relationship between orientation selectivity and ocular dominance. Journal of Physiology, 278, $27-44$.

GABOR, D. (1946). Theory of communication. IEEE, Transactions, 93 , 429-457. 
GiBson, J. J. (1950). Perception of the visual world. Boston: Houghton Mifflin.

GoGEL, W. C. (1956). The tendency to see objects as equidistant and its reverse relations to lateral separation. Psychological Monograph, 70(Whole No. 411).

GoGeL, W. C. (1965). Equidistance tendency and its consequences. Psychological Bulletin, 64, 153-163.

GoGEL, W. C. (1970). The adjacency principle and three-dimensional visual illusions. Psychonomic Monograph Supplement, 3(Whole No. 45), 153-169.

Graham, N. (1981). The visual system does a crude Fourier analysis of patterns. In S. Grossberg (Ed.), Mathematical psychology and psychophysiology. Providence, RI: American Mathematical Society.

Graham, N., NACHmias, J. (1971). Detection of grating patterns containing two spatial frequencies: A test of single-channel and multiple channel models. Vision Research, 11, 251-259.

Grossberg, S. (1968). Some physiological and biochemical consequences of psychological postulates. Proceedings of the National Academy of Sciences, 60, 758-765.

Grossberg, S. (1969). On learning and energy-entropy dependence in recurrent and nonrecurrent signed networks. Journal of Statistical Physics, 1, 319-350.

Grossberg, S. (1976a). Adaptive pattern classification and universal recoding: I. Parallel development and coding of neural feature detectors. Biological Cybernetics, 23, 121-134.

GrossBerg, S. (1976b). Adaptive pattern classification and universal recoding: II. Feedback, expectation, olfaction, and illusions. Biological Cybernetics, 23, 187-202.

Grossberg, S. (1978). A theory of human memory: Self-organization and performance of sensory-motor codes, maps, and plans. In R. Rosen \& F. Snell (Eds.), Progress in theoretical biology (Vol. 5). New York: Academic Press.

Grossberg, S. (1980). How does a brain build a cognitive code? Psychological Review, 87, 1-51.

Grossberg, S. (1981). Adaptive resonance in development, perception, and cognition. In S. Grossberg (Ed.), Mathematical psychology and psychophysiology. Providence, RI: American Mathematical Society.

GrossBerg, S. (1983a). Neural substrates of binocular form perception: Filtering, matching, diffusion, and resonance. In E. Basar, H. Flohr, H. Haken, \& A. J. Mandell (Eds.), Symergetics of the brain. New York: Springer-Verlag.

Grossberg, S. (1983b). The quantized geometry of visual space: The coherent computation of depth, form, and lightness. Behavioral \& Brain Sciences, 6, 625-692.

Grossberg, S. (1984). Outline of a theory of brightness, color, and form perception. In E. Degreef \& J. van Buggenhaut (Eds.), Trends in mathematical psychology. Amsterdam: North-Holland.

GrossBerg, S. (1987a). The adaptive brain: II. Vision, speech, language, and motor control. Amsterdam: North-Holland.

Grossberg, S. (1987b). Cortical dynamics of three-dimensional form, color, and brightness perception: I. Monocular theory. Perception \& Psychophysics, 41, 87-116.

Grossberg, S., \& LeVINE, D. S. (1975). Some developmental and attentional biases in the contrast enhancement and short term memory of recurrent neural networks. Journal of Theoretical Biology, 53, 341-380.

Grossberg, S., \& MArshall, J. (1987). A computational model of how cortical complex cells multiplex information about position, contrast, orientation, spatial frequency, and disparity. Manuscript in preparation.

Grossberg, S., \& Mingolla, E. (1985a). Neural dynamics of form perception: Boundary completion, illusory figures, and neon color spreading. Psychological Review, 92, 173-211.

Grossberg, S., \& Mingolla, E. (1985b). Neural dynamics of perceptual grouping: Textures, boundaries, and emergent segmentations. Perception \& Psychophysics, 38, 141-171.

Grossberg, S., * Mingolla, E. (1986). Computer simulation of neural networks for perceptual psychology. Behavior Research Methods, Instruments, \& Computers, 18, 601-607.

Grossberg, S., Mingolla, E. (1987). Neural dynamics of surface perception: Boundary webs, illuminants, and shape-from-shading. Computer Vision, Graphics, \& Image Processing, 37, 116-165.

HebB, D. O. (1949). The organization of behavior. New York: Wiley. Hering, E. (1964). Outlines of a theory of the light sense. Cambridge, MA: Harvard University Press.

Hirsch, H. V. B., Spinelu, D. N. (1970). Visual experience modifies distribution of horizontally and vertically oriented receptive fields in cats. Science, 168, 869-871.

Hollins, M., \& BalleY, G. W. (1981). Rivalry target luminance does not affect suppression depth. Perception \& Psychophysics, 30, 201-203.

HubeL, D. H., Wiesel, T. N. (1962). Receptive fields, binocular interaction and functional architecture in the cat's visual cortex. Joumal of Physiology, 160, 106-154.

HuBEL, D. H., \&iesel, T. N. (1968). Receptive fields and functional architectures of monkey striate cortex. Journal of Physiology, 195, 215-243.

Hubel, D. H., Wiesel, T. N. (1970). Stereoscopic vision in macaque monkey. Nature, 225, 41-42.

Hubel, D. H., WIESEL, T. N. (1977). Functional architecture of macaque monkey visual cortex. Proceedings of the Royal Society of London (B), 198, 1-59.

Johansson, G. (1973). Visual perception of biological motion and a model for its analysis. Perception \& Psychophysics, 14, 201-211.

Johansson, G. (1975, June). Visual motion perception. Scientific American, 76-88.

JoHANSson, G. (1978). About the geometry underlying spontaneous visual decoding of the optical message. In E. L. J. Leeuwenberg \& H. F. J. M. Buffart (Eds.), Formal theories of visual perception. New York: Wiley.

JuLEsz, B. (1971). Foundations of cyclopean perception. Chicago: University of Chicago Press.

KanizsA, G. (1976). Subjective contours. Scientific American, 234, 48-52.

KanizsA, G. (1979). Organization in vision. New York: Praeger.

KAUfMAN, L. (1974). Sight and mind: An introduction to visual perception. New York: Oxford University Press.

King-Smith, P. E. (1975). Visual detection analysed in terms of luminance and chromatic signals. Nature, $255,69-70$.

KuLIKowskı, J. J. (1978). Limit of single vision in stereopsis depends on contour sharpness. Nature, 275, 126-127.

Kulikowsk,, J. J., KRanda, K. (1986). In J. D. Pettigrew, K. J. Sanderson, \& W. R. Levick (Eds.), Visual neuroscience. Cambridge: Cambridge University Press.

Kulikowski, J. J., Marčelua, S., \& Bishop, P. O. (1982). Theory of spatial position and spatial frequency relations in the receptive fields of simple cells in the visual cortex. Biological Cybernetics, 43, $187-198$.

Lee, B. B., Creutzfeldt, O. D., \& Elepfandt, A. (1979). The response of magno-and-parvocellular cells of the monkey's lateral geniculate body to moving stimuli. Experimental Brain Research, 35, 547-557.

LeGGe, G. E., \& Rubin, G. S. (1981). Binocular interactions in suprathreshold contrast perception. Perception \& Psychophysics, 30, 49-61.

Levelt, W. J. M. (1965). On binocular rivalry. Soesterberg, The Netherlands: Institute for Perception, RVO-TNO.

LeVenthal, A. G., \& Schall, J. D. (1983). Structural basis of orientation sensitivity of cat retinal ganglion cells. Journal of Comparative Neurology, 220, 465-475.

Levick, W. R., * ThiBos, L. N. (1982). Analysis of orientation bias in cat retina. Journal of Physiology, 329, 243-261.

LEvy, W. B. (1985). Associative changes at the synapse: LTP in the hippocampus. In W. B. Levy, J. Anderson, \& S. Lehmkuhle (Eds.), Synaptic modification, neuron selectivity, and nervous system organization (pp. 5-33). Hillsdale, NJ: Eribaum.

Levy, W. B., Brassel, S. E., \& Moore, S. D. (1983). Partial quantification of the associative synaptic learning rule of the dentate gyrus. Neuroscience, 8, 799-808.

Levy, W. B., Desmond, N. L. (1985). The rules of elemental syn- 
aptic plasticity. In W. B. Levy, J. Anderson, \& S. Lehmkuhle (Eds.), Synaptic modification, neuron selectivity, and nervous system organization (pp. 105-121). Hillsdale, NJ: Erlbaum.

Livingstone, M. S., \& Hubel, D. H. (1984a). Anatomy and physiology of a color system in the primate visual cortex. Journal of Neuroscience, 4, 309-356.

Livingstone, M. S., \& HuBeL, D. H. (1984b). Specificity of intrinsic connections in primate primary cortex. Journal of Neuroscience, 4, 2830-2835.

LU, C., \& FENDER, D. H. (1972). The interaction of color and luminance in stereoscopic vision. Investigative Ophthalmology, 11, 482-490.

MACKAY, D. M. (1957). Moving visual images produced by regular stationary patterns. Nature, 180, 849-850.

MACKAY, D. M., \& MACKAY, V. (1973). Orientation-sensitive aftereffects of dichoptically presented colour and form. Nature, 242, $477-479$.

MACKAY, D. M., \& MacKay, V. (1975). Dichoptic induction of McCollough-type effects. Quarterly Journal of Experimental Psychology, 27, 225-233.

MarR, D., Nishihara, H. K. (1978). Representation and recognition of the spatial organization of three-dimensional shapes. Proceedings of the Royal Society of London (B), 200, 269-294.

MARR, D., \& PoGgIo, T. (1976). Cooperative computation of stereo disparity. Science, 194, 283-287.

MARR, D., PoGglo, T. (1979). A computational theory of human stereo vision. Proceedings of the Royal Society of London (B), 204, 301-328.

McCArTer, A., \& Silver, A. I. (1977). The McCollough effect: A classical conditioning phenomenon? Vision Research, 17, 317-319.

McCollough, C. (1965). Color adaptation of edge-detectors in the human visual system. Science, 149, 1115-1116.

Metell, F. (1974). The perception of transparency. Scientific American, 230, 90-98.

Metelu, F., DA Pos, O., Cavedon, A. (1985). Balanced and unbalanced, complete and partial transparency. Perception \& Psychophysics, 38, 354-366.

Meyer, G. E., \& Senecal, M. (1983). The illusion of transparency and chromatic subjective contours. Perception \& Psychophysics, 34, 58-64.

Mullukin, W. H., Jones, J. P., \& Palmer, L. A. (1984). Periodic simple cells in cat area 17. Journal of Neurophysiology, 52, 372-387.

MURCH, G. M. (1972). Binocular relationships in a size and color orientation specific aftereffect. Journal of Experimental Psychology, 93, 30-34.

Poggio, G. F., Motter, B. C., SQuatrito, S., \& Trotter, Y. (1985). Responses of neurons in visual cortex (V1 and V2) of the alert macaque to dynamic random-dot stereograms. Vision Research, 25, $397-406$.

Pollen, D. A., Andrews, B. W., \& Feldon, S. E. (1978). Spatial frequency selectivity of periodic complex cells in the visual cortex of the cat. Vision Research, 18, 665-682.

Pollen, D. A., \& Ronner, S. F. (1975). Periodic excitability changes across the receptive fields of complex cells in the striate and parastriate cortex of the cat. Journal of Physiology, 245, 667-697.

Pollen, D. A., Ronner, S. F. (1981). Phase relationships between adjacent simple cells in the visual cortex. Science, 212, 1409-1411.

Pollen, D. A., \& RonNer, S. F. (1983). Visual cortical neurons as localized spatial frequency filters. IEEE Transactions on Systems, Man, and Cybernetics, SMC-13, 907-916.

RAmachandRan, V. S., \& Nelson, J. I. (1976). Global grouping overrides point-to-point disparities. Perception, 5, 125-128.

Rauschecker, J. P., Singer, W. (1979). Changes in the circuitry of the kitten's visual cortex are gated by postsynaptic activity. $\mathrm{Na}$ ture, 280, 58-60.

Redies, C., \& Spillmann, L. (1981). The neon color effect in the Ehrenstein illusion. Perception, 10, 667-681.

Regan, D., Be Beverey, K. I. (1979). Binocular and monocular stimuli for motion in depth: Changing-disparity and changing-size feed the same motion-in-depth stage. Vision Research, 19, 1331-1342.

Regan, D., \&YNADER, M. (1982). Neurons in cat visual cortex tuned to the direction of motion in depth: Effect of stimulus speed. Investigative Ophthalmology \& Visual Science, 22, 535-550.
Rockland, K. S., \& LuND, J. S. (1983). Intrinsic laminar lattice connections in primate visual cortex. Journal of Comparative Neurology, 216, 303-318.

Russell, P. W. (1979). Chromatic input to stereopsis. Vision Research, 19, 831-834.

SavoY, R. L. (1984). "Extinction" of the McCollough effect does not transfer interocularly. Perception \& Psychophysics, 36, 571-576.

SChwartz, B. J., \& Speruing, G. (1983). Luminance controls the perceived 3-D structure of dynamic 2-D displays. Bulletin of the Psychonomic Society, 21, 456-458.

SCHWARTZ, E. L. (1980). Computational anatomy and functional architecture of striate cortex: A spatial mapping approach to perceptual coding. Vision Research, 20, 645-669.

Shinkman, P. G., \& Bruce, C. J. (1977). Binocular differences in cortical receptive fields of kittens after rotationally disparate binocular experience. Science, 197, 285-287.

Sillito, A. M. (1974). Modification of the receptive field properties of neurons in the visual cortex by bicuculline, a GABA antagonist. Journal of Physiology, 239, 36P-37P.

Silurto, A. M. (1975a). The contribution of inhibitory mechanisms to the receptive field properties of neurones in the striate cortex of the cat. Joumal of Physiology, 250, 305-329.

SilurTo, A. M. (1975b). The effectiveness of bicuculline as an antagonist of GABA and visually evoked inhibition in the cat's striate cortex. Journal of Physiology, 250, 287-304.

SILLTTO, A. M. (1977). Inhibitory processes underlying the directional specificity of simple, complex and hypercomplex cells in the cat's visual cortex. Journal of Physiology, 271, 699-720.

SilurTo, A. M. (1979). Inhibitory mechanisms influencing complex cell orientation selectivity and their modification at high resting discharge levels. Journal of Physiology, 289, 33-53.

Sillito, A. M., SAlt, T. E., KeMP, J. A. (1985). Modulatory and inhibitory processes in the visual cortex. Vision Research, 25, 375-381.

SINGER, W. (1983). Neuronal activity as a shaping factor in the selforganization of neuron assemblies. In E. Basar, H. Flohr, H. Haken, \& A. J. Mandell (Eds.), Synergetics of the brain. New York: SpringerVerlag.

SINGER, W. (1985). Central control of developmental plasticity in the mammalian visual cortex. Vision Research, 25, 389-396.

Skowвo, D. (1984). Are McCollough effects conditioned responses? Psychological Bulletin, 96, 215-226.

SKOWBO, D., FoRSTER, T. (1983). Further evidence against the classical conditioning model of McCollough effects. Perception \& Psychophysics, 34, 552-554.

SKowbo, D., Timney, B. N., Gentry, T. A., \& Morant, R. B. (1975). McCollough effects: Experimental findings and theoretical accounts. Psychological Bulletin, 82, 497-510.

Skowвo, D., \& White, K. (1983). McCollough effect acquisition depends on duration of exposure to inducing stimuli, not number of stimulus presentations. Perception \& Psychophysics, 34, 549-551.

Smith, E. L., III, LeVI, D. M., HarWerth, R. S., \& White, J. M. (1982). Color vision is altered during the suppression phase of binocular rivalry. Science, 218, 802-804.

SPERLING, G. (1970). Binocular vision: A physical and a neural theory. American Journal of Psychology, 83, 461-534.

Tausch, R. (1953). Die beidäugige Raumwahrnehmung-ein Prozess auf Grund der Korrespondenz und Disparation von Gestalten anstelle der Korrespondenz oder Disparation einzelner Netzhautelemente. Zeitschrift für Experimentelle und Angewandte Psychologie, 1, 394-421.

ThorelL, L. G., De VAloIs, R. L., \& Albrecht, D. G. (1984). Spatial mapping of monkey V1 cells with pure color and luminance stimuli. Vision Research, 24, 751-769.

vaN TUut, H. F. J. M. (1975). A new visual illusion: Neonlike color spreading and complementary color induction between subjective contours. Acta Psychologica, 39, 441-445.

van Tuiul, H. F. J. M., \& DE Weert, C. M. M. (1979). Sensory conditions for the occurrence of the neon spreading illusion. Perception, 8, 211-215.

VidYASAGAR, T. R. (1976). Orientation specific colour adaptation at a binocular site. Nature, 261, 39-40.

Vidyasagar, T. R., \& URBas, J. V. (1982). Orientation sensitivity of 
cat LGN neurones with and without inputs from visual cortical areas 17 and 18. Experimental Brain Research, 46, 157-169.

von Der Heydt, R., HÄNNY, P., Dürsteler, M. R. (1981). The role of orientation disparity in stereoscopic perception and the development of binocular correspondence. In E. Grastyán \& P. Molnár (Eds.), Advances in physiological science: Vol. 16. Sensory functions. Elmsford, NY: Pergamon Press.

voN TSCHERMAK-SEYSENEGG, A. (1952). Introduction to physiological optics (P. Boeder, Trans.). Springfield, IL: Thomas.

WALES, R., FoX, R. (1970). Incremental detection thresholds during binocular rivalry suppression. Perception \& Psychophysics, 8, 90-94.

Werner, H. (1937). Dynamics in binocular depth perception. Psychological Monograph (Whole No. 218).

White, K. D., Petry, H. M., Riggs, L. A., \& Miller, J. (1978). Binocular interactions during establishment of McCollough effects. Vision Research, 18, 1201-1215.
Wilde, K. (1950). Der Punktreiheneffekt und die Rolle der binocularen Querdisparation beim Tienfenshen. Psychologische Forschung, 23, 223-262.

Willshaw, D. J., \& von der Malsburg, C. (1976). How patterned neural connections can be set up by self-organization. Proceedings of the Royal Society of London $(B), 194,431-445$.

ZEKI, S. (1983a). Colour coding in the cerebral cortex: The reaction of cells in monkey visual cortex to wavelengths and colours. Neuroscience, 9, 741-765.

ZEKI, S. (1983b). Colour coding in the cerebral cortex: The responses of wavelength-sensitive and colour coded cells in monkey visual cortex to changes in wavelength composition. Neuroscience, 9, 767-791.

(Manuscript received December 19, 1985;

revision accepted for publication November 4, 1986.) 